\title{
A Reduced Power, 6-Tap Programmable Pre-Emphasis Circuit
}

\author{
by \\ Dezhong Cheng \\ B.Sc. M. Sc. \\ A Thesis submitted to \\ the Faculty of Graduate Studies and Research \\ in partial fulfilment of \\ the requirements for the degree of \\ Master of Applied Science in Electrical Engineering
}

Ottawa-Carleton Joint Institute for Electrical and Computer Engineering

Department of Electronics

Carleton University

Ottawa, Ontario, Canada

August 2009

Copyright (C)

2009 - Dezhong Cheng 


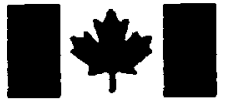

Library and Archives

Canada

Published Heritage

Branch

395 Wellington Street

Ottawa ON K1A ON4

Canada
Bibliothèque et

Archives Canada

Direction du

Patrimoine de l'édition

395, rue Wellington

Ottawa ON K1A ON4

Canada
Your file Votre refference

ISBN: 978-0-494-60254-6

Our file Notre référence

ISBN: 978-0-494-60254-6
NOTICE:

The author has granted a nonexclusive license allowing Library and Archives Canada to reproduce, publish, archive, preserve, conserve, communicate to the public by telecommunication or on the Internet, loan, distribute and sell theses worldwide, for commercial or noncommercial purposes, in microform, paper, electronic and/or any other formats.

The author retains copyright ownership and moral rights in this thesis. Neither the thesis nor substantial extracts from it may be printed or otherwise reproduced without the author's permission.
AVIS:

L'auteur a accordé une licence non exclusive permettant à la Bibliothèque et Archives Canada de reproduire, publier, archiver, sauvegarder, conserver, transmettre au public par télécommunication ou par l'Internet, prêter, distribuer et vendre des thèses partout dans le monde, à des fins commerciales ou autres, sur support microforme, papier, électronique et/ou autres formats.

L'auteur conserve la propriété du droit d'auteur et des droits moraux qui protège cette thèse. $\mathrm{Ni}$ la thèse ni des extraits substantiels de celle-ci ne doivent être imprimés ou autrement reproduits sans son autorisation.
In compliance with the Canadian Privacy Act some supporting forms may have been removed from this thesis.

While these forms may be included in the document page count, their removal does not represent any loss of content from the thesis.
Conformément à la loi canadienne sur la protection de la vie privée, quelques formulaires secondaires ont été enlevés de cette thèse.

Bien que ces formulaires aient inclus dans la pagination, il n'y aura aucun contenu manquant.

\section{Canadä}




\section{Important Information}

The information used in this thesis comes in part from the research program of Dr. Tadeusz Kwasniewski and his associates in the VLSI in Communication Group at Carleton University. The research results appearing in this thesis represent an inte-

gral part of the ongoing research program. All research results in this thesis including tables, graphs, and figures, but excluding the narrative portions of the thesis are effectively incorporated into the research program and can be used by Dr. Tadeusz Kwasniewski and his associates for educational and research purposes, including publication in open literature with appropriate credits. The matters' intellectual property may be pursued cooperatively with Carleton University and Dr. Tadeusz Kwasniewski, when and where appropriate. 


\section{List of Symbols}

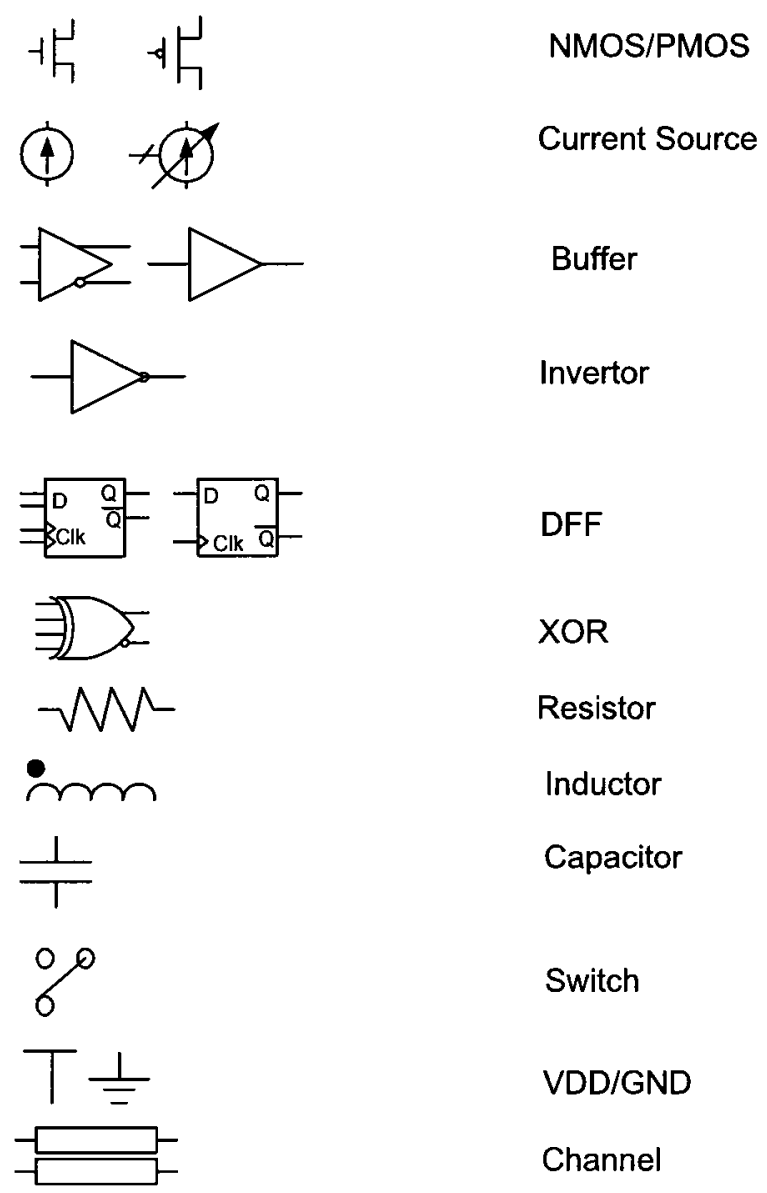




\section{List of Abbreviations}

$\begin{array}{ll}\text { AC } & \text { Alternating Current } \\ \text { ADC } & \text { Analog to Digital Convertor } \\ \text { WPE } & \text { Well Proximity Effect } \\ \text { BCE-NRZ } & \text { Bit Center NRZ Equalizer } \\ \text { BEE-NRZ } & \text { Bit Edge NRZ Equalizer } \\ \text { BER } & \text { Bit-Error Rate } \\ \text { BW } & \text { Bandwidth } \\ \text { CEI } & \text { Common Electronic Interface } \\ \text { CFT } & \text { Continuous Fourier Transform } \\ \text { CDR } & \text { Clock and Data Recovery } \\ \text { CM } & \text { Current Mirror } \\ \text { CML } & \text { Current Mode Logic } \\ \text { CMOS } & \text { Complementary Metal-Oxide-Semiconductor } \\ \text { CMU } & \text { Clock Management Unit } \\ \text { DEMUX } & \text { Demultiplexer } \\ \text { DFE } & \text { Decision Feedback Equalizer } \\ \text { DC } & \text { Direct Current } \\ \text { DFF } & \text { D Flip-Flop } \\ \text { DUT } & \text { Device Under Test } \\ \text { FFE } & \text { Feed Forward Equalizer } \\ \text { FIR } & \text { Finite Impulse Response } \\ \text { FSF } & \text { Fraction Space FIR } \\ \end{array}$




\begin{tabular}{|c|c|}
\hline Gbps & Giga Bit-Per-Second \\
\hline $\mathrm{I} / \mathrm{O}$ & Input/Output \\
\hline IC & Integrated Circuit \\
\hline ISI & Intersymbol Interference \\
\hline iDAC & Digital to Analog Converter Current Source \\
\hline JTAG & Joint Test Action Group \\
\hline LMS & Least Mean Squares \\
\hline LSB & Least Significant Bit \\
\hline MOSFET & Metal Oxide Semiconductor Field Effect Transistor \\
\hline MSB & Most Significant Bit \\
\hline MUX & Multiplexer \\
\hline NMOS & N-Channel MOSFET \\
\hline NRZ & Non-Return-to-Zero \\
\hline PAM & Pulse Amplitude Modulation \\
\hline PCB & Printed Circuit Board \\
\hline PMOS & P-Channel MOSFET \\
\hline PISO & Parallel In and Serial Out \\
\hline PRBS & Pseudorandom Binary Sequence \\
\hline PVT & Process, Voltage, Temperature \\
\hline SCR & Silicon Control Rectifier \\
\hline SerDes & Serializer/Deserializer \\
\hline SIPO & Serial In and Parallel Out \\
\hline SNR & Signal-to-Noise Ratio \\
\hline SSF & Symbol Space FIR \\
\hline STDC & Single to Differential Converter \\
\hline TQFP & Thin Quad Flat Pack \\
\hline $\mathbf{V}_{T}$ & Threshold Voltage \\
\hline $\mathbf{Z F}$ & Zero Force \\
\hline
\end{tabular}




\section{Abstract}

This thesis starts with the motivation of the research and a review of the techniques to solve the inter-symbol interference (ISI) problem caused by the limited bandwidth of the channel for high data rate digital communication.

Pre-emphasis is often employed at the transmitter side to counteract the ISI in high-speed digital data communications. Traditional pre-emphasis drivers, implemented in CML, use one pair of CMOS transistors at the output stage. To design a pre-emphasis circuit for different channels or for the same channel using different types of equalizers requires a wide range of current for the same tap. The challenge for traditional circuits is how to choose the sizes for these transistors. To meet this

challenge, this paper presents a low power, 6-tap pre-emphasis circuit with several pairs of transistors for each tap at output stage to solve this issue.

To verify this design method, a 6-tap pre-emphasis circuit with programmable coefficients was designed using 90nm CMOS technology. The schematic with post layout simulation has shown that the eye diagram was open at all PVT corners. The test results indicate that the coefficients can be made programmable through the digital-to-analog converter current source. The maximum relative error is $9.62 \%$ when Bit 2 of tap 3 is set to one and all other bits of tap 3 and all bits of other taps are set to zero. 


\section{Acknowledgments}

I would like to thanks everyone that has enabled me to complete this thesis.

I am deeply grateful to my supervisor, Dr. Tadeusz Kwasniewski. I would like to thank him for giving me advisor to accomplish the research work both in technology and life. Dr. Kwasniewski is always there for help when I was trapped in some problems.

I also want to express my thanks to co-supervisor Dr. David Chen. He always explained any question I had in very detail.

I would also like to thank my course instructors, Dr. Calvin Plett, Dr. Len MacEchern, Dr. Ralph Mason and Dr. John W. M. Rogers for their instructions in the courses I took. I wish to thank all the staff of DOE for their great assistances. I would to thank Nagui Mikhail for his preparing the test equipment in the test stage.

I am very grateful to Bangli Liang, Bo Wang, Lei Zhang, M. Usama, Irfan, Ramin, and other group members for being great colleagues and friends. I wish to thank all the people in VLSI team for all the help and assistance.

I wish to thank my wife Xiaoming Huang, my mom, and my sons Jerry and William for their supporting me to finish the study, giving me the reason to be happy, and strength to work and live. Each in their own way gave me the strength to stay on the course, and for that I dedicate this thesis to them. 


\section{Table of Contents}

Preface $\quad$ iii

List of Symbols $\quad$ iv

List of Abbreviations $\quad$ v

Abstract vii

Acknowledgments viii

Table of Contents $\quad$ ix

List of Figures $\quad$ xii

1 Introduction $\quad 1$

1.1 Research Motivation . . . . . . . . . . . . . . . 1

1.2 Thesis Objective ....................... 3

1.3 Thesis Outline . . . . . . . . . . . . . . . . . . . 4

2 Equalizer Technology Review 5

2.1 Chapter Overview ...................... 5

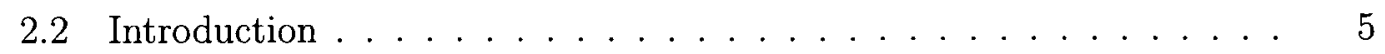

2.3 Transmission Loss and Group Delay . . . . . . . . . . . . . . . 8

2.4 Feed Forward Equalizer $(\mathrm{FFE}) \ldots \ldots \ldots 11$ 
2.4.1 Bit-center NRZ Equalizer (BCE-NRZ) . . . . . . . . 13

2.4 .2 Bit Edge Equalizer (BEE-NRZ) . . . . . . . . . . 14

2.4 .3 Duobinary . . . . . . . . . . . . . . 15

2.5 Decision Feedback Equalizer $(\mathrm{DFE}) \ldots \ldots \ldots \ldots$

2.6 Chapter Summary . . . . . . . . . . . . . . . . . 18

3 Pre-Emphasis Design at the System Level 19

3.1 Chapter Overview . . . . . . . . . . . . . . . . . . 19

3.2 Theory of FFE in the Digital Circuit . . . . . . . . . . . . 19

3.3 Optimizing Coefficients of the FIR Filter . . . . . . . . . . . 21

3.4 Proposed FIR Pre-emphasis Architecture . . . . . . . . . . . . . . 23

3.5 Behavioral Model and Verification . . . . . . . . . . . . 24

3.6 Chapter Summary . . . . . . . . . . . . . . . 25

4 Design of the FIR Pre-emphasis Circuit in 90nm CMOS Technology 28

4.1 Chapter Overview . . . . . . . . . . . . . . . . 28

4.2 FIR Pre-emphasis Circuit Block Diagram . . . . . . . . . . . . . 28

4.3 Current Mirror (CM) f . . . . . . . . . . . . . 32

4.4 DFF, XOR, Buffer and Mux . . . . . . . . . . . 36

4.5 Pre-emphasis Driver Circuit . . . . . . . . . . . . . . . . 38

4.5.1 Sizing Transistors in the Output Stage . . . . . . . . . 38

4.5 .2 iDAC Circuit . . . . . . . . . . . . . . . 43

4.6 Chip Interface and Memory Circuits _ . . . . . . . . . . . . 45

4.7 Single to Differential Converter (STDC) . . . . . . . . . . . 46

4.8 Layout . . . . . . . . . . . . . . . . . . . . 46

4.9 Schematic and Post-Layout Simulation . . . . . . . . . . 48

4.10 Chapter Summary . . . . . . . . . . . . . . . . 52 
5 Test Setup and Measurement Results $\quad 54$

5.1 Introduction . . . . . . . . . . . . . . . . 54

5.2 Test Bench Setup . . . . . . . . . . . . . . . 54

5.3 Measurement Results . . . . . . . . . . . . . . . . . 55

6 Conclusion, Contribution and Future Work $\quad 59$

6.1 Conclusions . . . . . . . . . . . . . . . . . . 59

6.2 Contribution . . . . . . . . . . . . . . 60

6.3 Future Work . . . . . . . . . . . . . . . . . 60

6.4 Publications ........................... 61

$\begin{array}{ll}\text { List of References } & 63\end{array}$

$\begin{array}{ll}\text { Appendix A Schematic of Pre-emphasis Circuits } & 68\end{array}$

$\begin{array}{lll}\text { Appendix B Layout of Pre-emphasis Circuits } & 82\end{array}$ 


\section{List of Tables}

2.1 Normalized 1 V Pulse Response at Different Data Rates . . . . . . 8

2.2 Comparison of Eye Opening of Different Equalizers _ . . . . . . . . 17

3.1 Coefficients for Channel B20 in Different Models Using VerilogA Blocks 26

4.1 Representative Sample of 6-Bit Signed Digital Code and Current Relationship . . . . . . . . . . . . . . . . . . . 31

4.2 Traditional Circuit and Proposed Circuit Capacitance Comparison . . 41

4.3 Schematic Simulation Results _ . . . . . . . . . . . . . . . . . 49

4.4 Post Layout Simulation Results . . . . . . . . . . . . . . . 52

4.5 Power Dissipation Comparison . . . . . . . . . . . . . . 52

5.1 Measured Differential Output Signal Value vs Coefficients Value . . . 57 


\section{List of Figures}

1.1 Typical Backplane Topology . . . . . . . . . . . . . . . . 2

2.1 Block Diagram of a Popular Backplane Transceiver. . . . . . . . 6

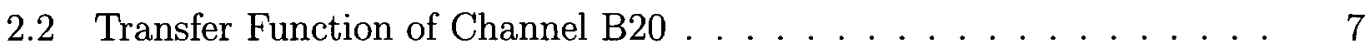

$2.3 \quad 1$ V Pulse Response at Different Baud Rates for Channel B20 $\ldots .7$

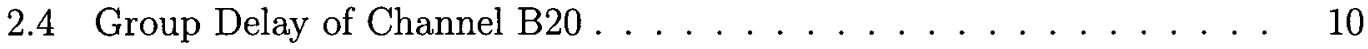

2.5 Architecture of FIR Filter $\ldots \ldots \ldots \ldots \ldots \ldots \ldots$

2.6 Eye Diagram of B20 for 10 Gbps (BCE) $\ldots \ldots \ldots \ldots \ldots$

2.7 Eye Diagram of B20 for 10 Gbps (BEE) $\ldots \ldots \ldots \ldots \ldots$

2.8 Eye Diagram of B20 for 10 Gbps (Duobinary) $\ldots \ldots \ldots \ldots \ldots$

3.1 An Analytical Model of a Transceiver . . . . . . . . . . . 20

3.2 Architecture of the Proposed FIR Filter $\ldots \ldots \ldots \ldots \ldots$

3.3 Test Bench of FIR Filter (VerilogA Model) . . . . . . . . . 25

3.4 Eye Diagram of B20 for Differential Equalizers Using VerilogA Model 27

4.1 Proposed FIR Pre-emphasis Circuit Block Diagram . . . . . . . . 29

4.2 Comparison of Current for CMOS Rail-to-Rail and CML circuit Vs Frequency . . . . . . . . . . . . . . . . . . 29

4.3 Top Level of the 6-Tap FIR Filter . . . . . . . . . . . . . 30

4.4 Different Current Mirror Circuits . . . . . . . . . . . . . 32

4.5 Comparison Performance of Different Types of Current Mirror . . . 35

4.6 D-Latch, XOR, Buffer and MUX in CML . . . . . . . . . 36 
4.7 $f_{T}$ vs Current Density and $V_{g s}$ (NMOS W x L $=10 \mu \mathrm{m} \times 0.1 \mu \mathrm{m}, 90 \mathrm{~nm}$

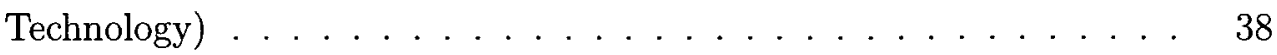

4.8 One Tap Pre-emphasis Driver . . . . . . . . . . . . . . . . . . . . 39

$4.9 C_{g s}$ and $C_{g d}$ vs Coefficients . . . . . . . . . . . 40

4.10 Error and Variance of the Output of a Current Source . . . . . . . . 42

4.11 Output of a Current Source at Different Coefficients . . . . . . . . 43

4.12 Voltage Drop through a $2 \mathrm{nH}$ Inductor at Different Coefficients . . . . 44

4.13 Pre-Emphasis Driver of One Tap . . . . . . . . . . . . . 45

4.14 DFFs Used to Store the Value of the Coefficients . . . . . . . . . 46

4.15 Single-to-Differential Signal Converter . . . . . . . . . . . . . . . . . 47

4.16 Layout View of the Die . . . . . . . . . . . . . . . 47

4.17 Test Bench for the Chip . . . . . . . . . . . . . . . . . 49

4.18 Schematic Simulation . . . . . . . . . . . . . 50

4.19 Post Layout Simulation Using Different Extraction Strategies . . . . . 51

5.1 Pin Arrangement of 8-Pin Probes ... . . . . . . . . 55

5.2 Test Bench Using Lab Equipment . . . . . . . . . . . . . . . . . 55

5.3 Chip Test In the Lab . . . . . . . . . . . . . . 56

5.4 Test Result of the Output Signals . . . . . . . . . . . . . . . 58

A.1 Schematic of Buffer . . . . . . . . . . . . . 68

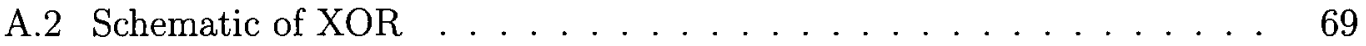

A.3 Schematic of $\mathrm{DFF} \ldots \ldots \ldots \ldots$. . . . . . . . . 70

A.4 Schematic of MUX . . . . . . . . . . . . . 71

A.5 Schematic of Current Mirror . . . . . . . . . . . . . 72

A.6 Schematic of Single-to-Differential Converter . . . . . . . . . . . . 73

A.7 Schematic of 2-Bit Driver f . . . . . . . . . . . 74

A.8 Schematic of 3-Bit Driver . . . . . . . . . . 75

A.9 Schematic of 4 -Bit Driver $\ldots \ldots \ldots \ldots \ldots$ 
A.10 Schematic of 5-Bit Driver f . . . . . . . . 77

A.11 Schematic of FIR . . . . . . . . . . . . 78

A.12 Schematic of Coefficients Storage . . . . . . . . . . . 79

A.13 Schematic of Pre-Emphasis Circuit . . . . . . . . . 80

A.14 Schematic of test bench of Pre-Emphasis Circuit . . . . . . . . 81

B.1 Layout of Single-to-Differential Converter and XOR . . . . . . . 82

B.2 Layout of Buffers . . . . . . . . . . . . . . . 83

B.3 Layout of DFFs . . . . . . . . . . . . . . . . 84

B.4 Layout of 2-Bit Driver . . . . . . . . . . . . 85

B.5 Layout of 3 -Bit Driver . . . . . . . . . . . . 86

B.6 Layout of 4-Bit Driver . . . . . . . . . . . . . 87

B.7 Layout of 5 -Bit Driver . . . . . . . . . . . . . . 87

B.8 Layout of Coefficients Storage . . . . . . . . . . . . . 88

B.9 Layout of Pre-Emphasis Circuit . . . . . . . . . . . . 89

B.10 Layout of Chip . . . . . . . . . . . . . . . . . . . . 89 


\section{Chapter 1}

\section{Introduction}

\subsection{Research Motivation}

Communication systems use either parallel bus topology or serial link topology. For long distance communications, optical fiber is efficient [1]. For short distance communication, such as chip-to-chip and board-to-board, optical fiber is too expensive. Traditional methods for short distance communication using parallel buses in chipto-chip or board-to-board communications require many parallel wires and are costly as well as power inefficient. Therefore high speed serial links are the preferred choice on Printed Circuit Boards (PCB) or backplanes for these applications. Recently, the need to transport high volumes of data from chip-to-chip, from board-to-board or from computer-to-computer through backplanes requires that the reduced I/O pin count high-speed Serializer/Deserializer (SERDES) replace the traditional conventional low-speed parallel bus structures. A typical topology of a modular platform backplane is shown in Figure 1.1. It consists of two daughter boards, on which the transceivers are located, and one backplane, which connects the two daughter boards. The daughter boards and backplane are attached by the connectors.

Products using multi gigabit-per-second (Gbps) data rates are already in the market. The standards for 10 Gbps communication have been released for many years, 


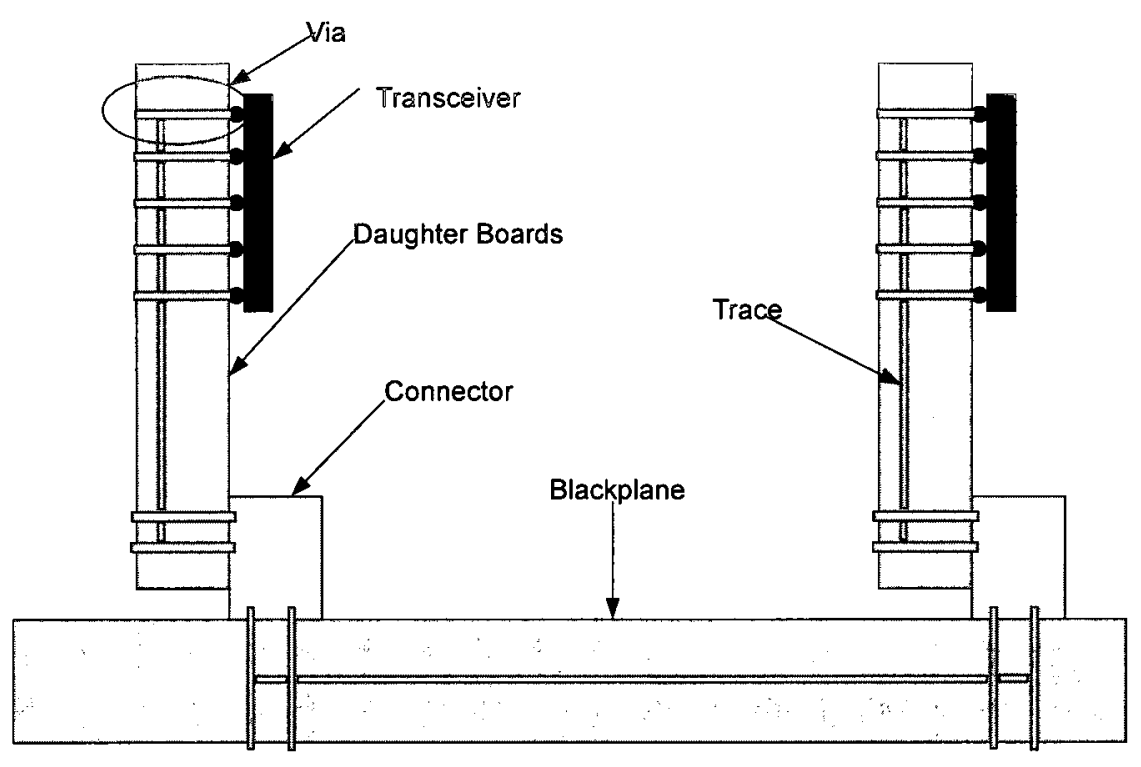

Figure 1.1: Typical Backplane Topology

such as Common Electrical I/O (CEI) 2.0 [2] and IEEE 802.3ap [3]. Now engineers on the IEEE P802.3ba Ethernet Task Force are discussing the next generation of communication at $40 \mathrm{Gbps}$ or $100 \mathrm{Gbps}$ [4]. However, the current backplane materials and connectors do not provide adequate bandwidth to support these transmission rates. There are several approaches being pursed by many vendors to increase the backplane transmission speed. These technologies fall into two basic categories. Upgrading the entire system to meet the high-speed requirements is an obvious but expensive way to solve the bandwidth problem. For example, updating the communication equipment from $1 \mathrm{G}$ to $2 \mathrm{G}$ costs a lot of money. Another more economical way is the use of signal processing to overcome the poor transmission properties of the physical channels.

Serial data systems operating at $10 \mathrm{Gbps}$ and beyond have a variety of components that determine system performance. These components include Inter-Symbol Interference (ISI), jitter, crosstalk, device noise and environment noise. In addition, channels are often severely band-limited and thus require the use of equalization [1]. 
One equalization technique focuses entirely on amplitude ISI at the bit center is called Bit-Center NRZ Equalization (BCE-NRZ). Another equalization technique is based on equalizing the edges of the data bits is called Bit-Edge NRZ Equalization (BEE-NRZ).

The equalizers can be implemented at the transmitter side, receiver side or both sides for different applications. The equalizer implemented at the transmitter side is often called pre-emphasis. The Finite Impulse Response (FIR) filter is widely used to implement the pre-emphasis. The inputs to this filter are the present, past and possibly future symbols. The coefficients of the filter depend on the channel characteristics. The length of the filter (number of filter taps) depends on the number of symbols that affect the present symbol. Therefore, the output of the filter is the present symbol value plus the weighted values of the $\mathrm{N}$ symbols affecting the current transmitted or future transmitted symbols.

\subsection{Thesis Objective}

The goal of this thesis is to design a reduced power, 6-tap pre-emphasis circuit using an FIR filter which can operate for BCE-NRZ, BEE-NRZ, duobinary or other algorithms as long as the architecture of the pre-emphasis circuit can be implemented as an FIR filter. Changing the weight of each tap is achieved by changing the coefficients stored in the chip therefore programmable coefficients are required. The pre-emphasis circuit operates for different channels or different algorithms by changing the coefficients through either the JATG interface or simple interface circuits as shift registers when the chip is initialized. The maximum data rate is $10 \mathrm{Gbps}$. The challenge in this design is to ensure that the designed pre-emphasis circuit operates for different types of algorithms, i.e. BCE-NRZ, BEE-NRZ and duobinary equalizers for different applications, such as differential channels and/or different data rates. This requires 
that the coefficients can vary widely for different applications. Changing coefficients means changing the current of relative tap and therefore changing the current density if the same width of the transistor is used. Selecting the size of the transistors is a challenge for designing an equalizer for a wide variety of applications.

\subsection{Thesis Outline}

This thesis is organized into the following chapters:

Chapter 1 is the introduction of the thesis including the research motivation, the objective and organization.

Chapter 2 reviews the background theory and different algorithms to account for the ISI problem due to the bandwidth limitation of the channel in both the wire line and optical communication systems.

Chapter 3 provides the theory and methodology to optimize the coefficients of the FIR filter.

Chapter 4 describes the detail design issue in $90 \mathrm{~nm}$ technology, including the circuits of different blocks. It coves the re-timing circuit, pre-drive circuits and driver circuit. The layout of the circuit in a $90 \mathrm{~nm}$ ST Microelectrons process is also discussed in this chapter.

Chapter 5 gives the test procedure and results.

Chapter 6 presents the conclusion of the research, outlines the contribution, list the resulting publications and offers a direction for future research work. 


\section{Chapter 2}

\section{Equalizer Technology Review}

\subsection{Chapter Overview}

In this chapter, the background of the equalization is discussed first. Then a review of several papers published in recent years regarding the different types equalizers is presented.

\subsection{Introduction}

Figure 2.1 shows the popular architecture of the transceiver in backplane applications [5]. For different applications, the transceiver may not contain all blocks as shown in Figure 2.1. For example, some transceivers may only have an equalizer at the transmitter side or some transceivers may only have an equalizer at the receiver side.

A transceiver used in a backplane is a wide-band system. However, the backplane does not provide sufficient bandwidth for multi-gigahertz or high data rates. Figure 2.2 shows the transfer function of channel B20. The specification of channel B20 is available at the IEEE 802.3ap web site [6]. In Figure 2.2, it shows that the channel has more attenuation at high frequencies than that at low frequencies. The attenuation 


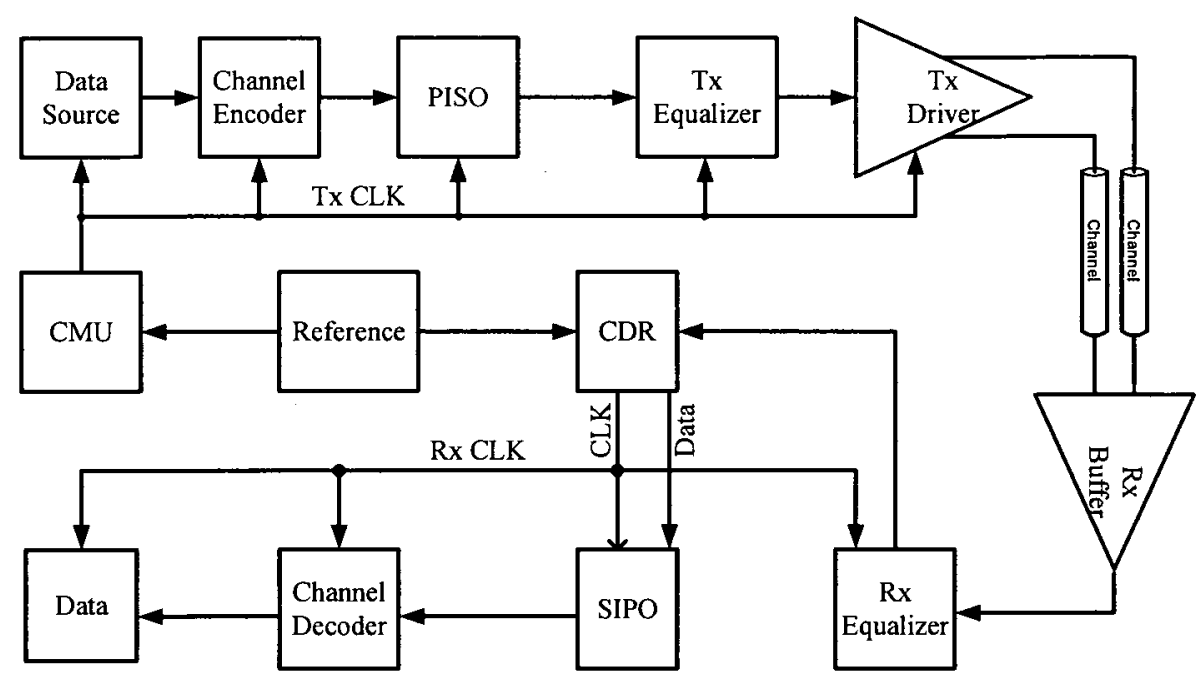

Figure 2.1: Block Diagram of a Popular Backplane Transceiver.

of the channel is $-18 \mathrm{~dB}$ and $-32 \mathrm{~dB}$ at $5 \mathrm{GHz}$ and $10 \mathrm{GHz}$ respectively. Therefore when a rectangular signal with one baud rate period travels through the backplane, the different frequencies contained in the pulse experience different energy loss and different delay. The high frequency signals lose more energy than the low frequency signals. As a consequence, its amplitude is attenuated and the width of its timedomain waveform is expanded into its neighbor symbols. This is one of the causes of ISI. As the data rate increases, one baud rate period signal expands into more than one period of neighbors. Figure 2.3 shows a $1 \mathrm{~V}$ pulse response of the channel B20 at different baud rates. In the Figure 2.3 , the $\mathrm{x}$-axis is the normalized period of the data baud rates. From Figure 2.3, two points are obvious. One is that the response with higher date rate has a longer tail on both sides of its peak value. The reason for this is due to the expansion of one baud rate period signal with the higher data rate expands into more neighbor symbols than that of the one baud rate period signal with the low data rate. Another point is that the response wave of the higher date rate symbol has a lower peak amplitude. There are two reasons for this. One reason is that the energy of the high baud rate $1 \mathrm{~V}$ pulse is less than that in the low baud 


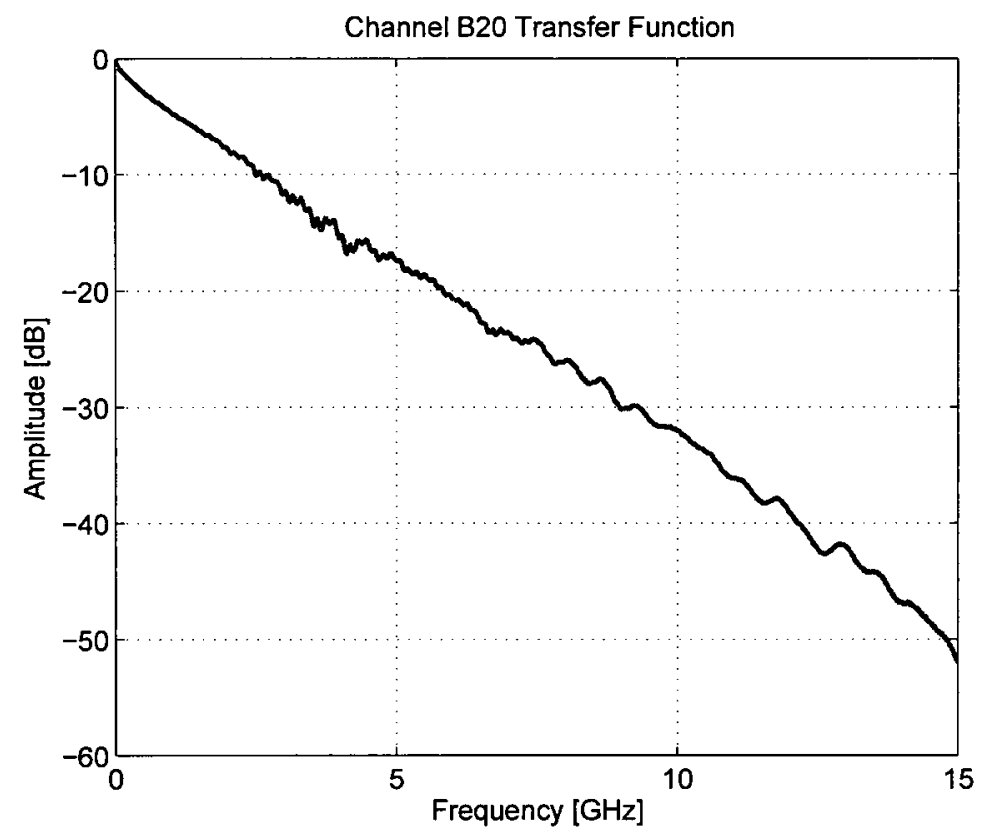

Figure 2.2: Transfer Function of Channel B20

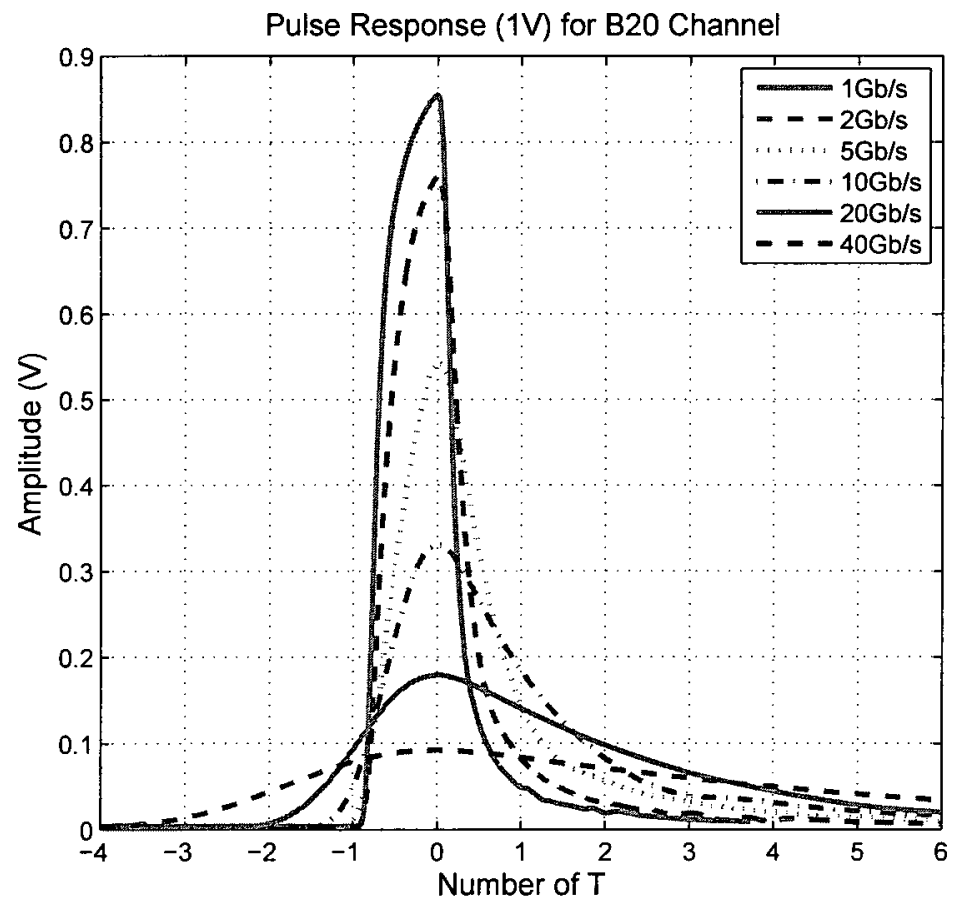

Figure 2.3: 1 V Pulse Response at Different Baud Rates for Channel B20 
Table 2.1: Normalized $1 \mathrm{~V}$ Pulse Response at Different Data Rates

\begin{tabular}{|c|c|c|c|c|c|c|c|c|c|c|c|}
\hline \multirow{2}{*}{$\begin{array}{c}\text { Data Rate } \\
\text { (Gbps) }\end{array}$} & \multicolumn{4}{|c|}{ Pre Tap } & \multirow{2}{*}{$\begin{array}{l}\text { Main } \\
\text { Tap }\end{array}$} & \multicolumn{6}{|c|}{ Post Tap } \\
\hline & 4 & 3 & 2 & 1 & & 1 & 2 & 3 & 4 & 5 & 6 \\
\hline 1 & 0.0037 & 0.0039 & 0.0036 & 0.0039 & 1 & 0.0566 & 0.0220 & 0.0134 & - & - & - \\
\hline 2 & 0.0019 & 0.0020 & 0.0014 & 0.0029 & 1 & 0.1089 & 0.0401 & 0.0241 & 0.0125 & 0.0111 & 0.0081 \\
\hline 5 & 0.0006 & 0.0023 & 0.0014 & 0.0165 & 1 & 0.2654 & 0.1042 & 0.0579 & 0.0365 & 0.0267 & 0.0180 \\
\hline 10 & 0.0012 & 0.0012 & 0.0009 & 0.1478 & 1 & 0.5591 & 0.2497 & 0.1192 & 0.0933 & 0.0636 & 0.0508 \\
\hline 20 & 0.0007 & 0.0005 & 0.0391 & 0.5167 & 1 & 0.7804 & 0.5449 & 0.3639 & 0.2468 & 0.1562 & 0.1084 \\
\hline 40 & 0.0241 & 0.1484 & 0.4752 & 0.8524 & 1 & 0.9068 & 0.7703 & 0.6481 & 0.5351 & 0.4428 & 0.3629 \\
\hline
\end{tabular}

rate $1 \mathrm{~V}$ pulse. Another reason is that the loss of energy of pulse is larger for the high baud rate pulse compared to the lower baud rate. The Table 2.1 shows the detailed values of the tail at integer periods, which is the normalization of the peak value to its own pulse response.

\subsection{Transmission Loss and Group Delay}

The frequency dependent transmission losses related to PCB layout are caused by the skin effect of the series resistive component of the copper and the dielectric loss due to the parallel conductive component of the dielectric materials [7].

Skin effect is the tendency of high frequency electric current to flow at the skin of the conductor. Skin effect causes the effective resistance of the conductor to increase as the frequency of the current increases. Dielectric loss is due to the property that the insulating materials between the conductors have different conductivity at different frequencies. Therefore part of the energy of the signals is absorbed by the insulating materials and the signals are attenuated once they arrive at the receiver side.

A backplane is essentially a collection of strip lines on PCBs that are mated via connectors. The strip line can be described with Equations (2.1) and (2.2) in 
transmission line theory.

$$
\begin{aligned}
& z=j 2 \pi f \mu_{0} g_{1}+2 g_{2} \sqrt{j \frac{2 \pi f \mu_{0}}{\sigma}} \\
& y=2 \pi f \varepsilon_{0}\left(j \varepsilon_{r}^{e f f}+\varepsilon_{r} \tan \delta\right) / g_{1}
\end{aligned}
$$

Where $z$ and $y$ are impedance per unit length and admittance per unit length, respectively, $g_{1}$ and $g_{2}$ are parameters decided by the geometries of the transmission line and their expressions are given in [8] and [9] respectively, $\varepsilon_{0}$ and $\mu_{0}$ are permittivity and permeability of free space, respectively, $\varepsilon_{r}$ is the relative permittivity of the dielectric material, $\varepsilon_{r}^{e f f}$ is the effective dielectric constant of the substrate, $\sigma$ is the conductivity of the strip, and $\tan (\delta)$ is the loss tangent of the substrate. The propagation constant $\gamma=\alpha+j \beta$ and the characteristic impedance $Z_{0}$ can be derived from Equations (2.1) and (2.2). At low frequencies, the skin effect dominates the attenuation factor $\alpha$ and it is approximately proportional to the square root of frequency. At high frequencies, the loss tangent of the substrate dominates $\alpha$ and it becomes approximately proportional to frequency [7].

The delay of signals depends on its frequency. The group delay is a measure of the travel time of a signal through a DUT versus frequency. In other words, the group delay is the time that it takes for the signals to travel through the DUT versus frequency. The data transmitted from the transmitter contains a wide range of frequencies, therefore, the waveforms containing different frequencies arrive at the receiver at different times, although they were sent at the same time [10]. Group delay is a very important parameter of phase distortion, and is calculated by differentiating the insertion response of the DUT versus frequency. Group delay is another factor causing distortion of the signals as the signals travel through backplane system. Group delay of the channel B20 is shown in Figure 2.4. From Figure 2.4, the delays at varying frequencies are different and has a range of $4.9 \mathrm{~ns}$ to $5.9 \mathrm{~ns}$. The maximum difference 
is $1 \mathrm{~ns}$ which is 10 data clock periods for a $10 \mathrm{Gbps}$ data rate.

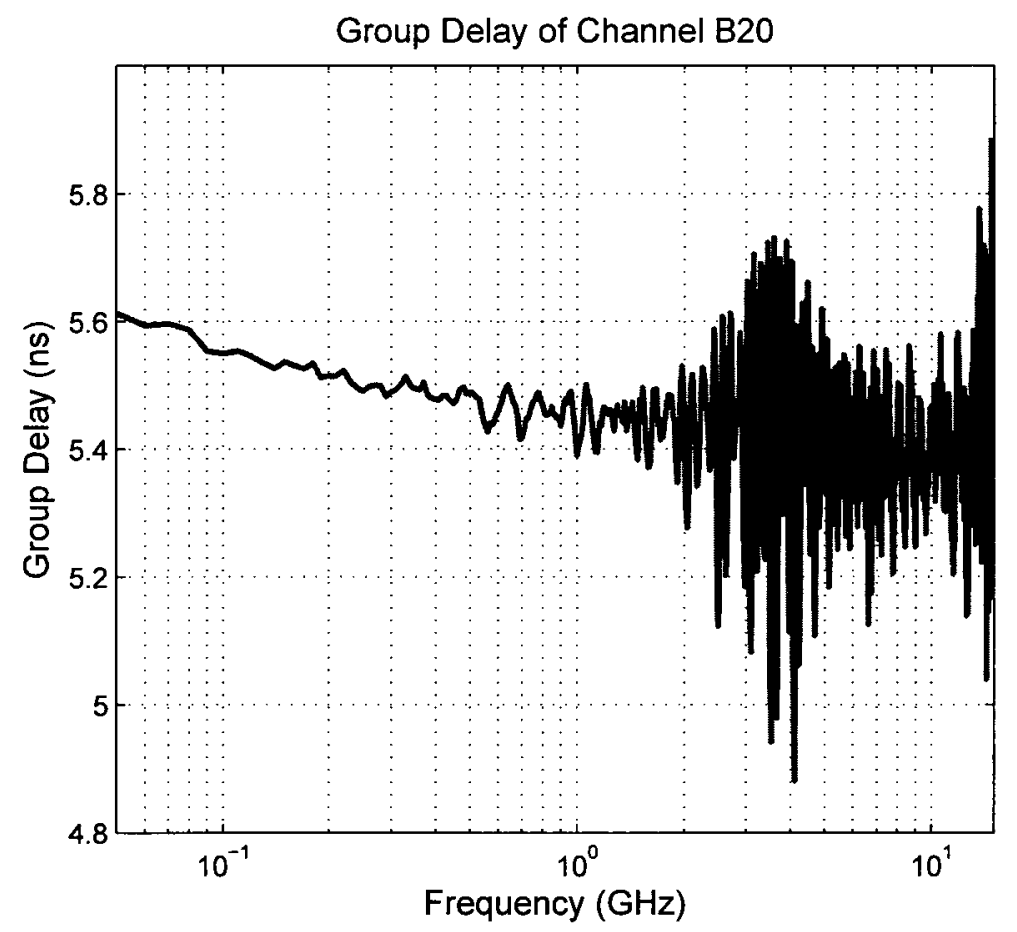

Figure 2.4: Group Delay of Channel B20

To reduce the ISI, one solution is to reduce the effective symbol rate, thereby reducing the required channel bandwidth, such as using 4-level pulse amplitude modulation (PAM-4) $[11,12]$ or duobinary signalling $[12,13]$. The spectra of duobinary and PAM-4 are both exactly half as wide as that of the NRZ data. The circuit for duobinary is simpler than the circuit for PAM-4. For this point view, duobinary signaling is superior to PAM-4 as it makes use of the intrinsic roll-off bandwidth of the channel as part of the desired transfer function [12]. PAM-4 can reduce the effective symbol rate by factor of 2 for the same data rate but the circuits are more complex and the signal to noise ratio (SNR) is smaller for the same maximum output amplitude. At the receiver, the received signals also are 4-level signals and as a result three eyes stack vertically. The amplitude of the eye opening is small for the same $V_{P P} \mathrm{Rx}$ 
signal compared to NRZ signals, which only has one eye. In addition, all level transitions in PAM-4 result in larger jitter and is another factor to limit its application [12]. The circuits for duobinary signalling at the receiver are also complex compared to the circuits for NRZ signaling. The duobinary detector circuits at the receiver are required to identify 3-level signals therefore the SNR of duobinary is better than that of PAM-4 but worse than NRZ assuming that the maximum amplitude of the receiver signals are the same for the three signaling types.

Using an equalizer is another popular way to solve the ISI problem. The circuits of an equalizer can be analog $[14,15]$ or digital $[10,16]$. The equalizer based on the digital circuit is discussed in this thesis. The equalizer can be linear $[10,17]$,

non-linear $[18-20]$ or both $[16,21]$. The linear equalizer can be implemented on the transmitter side $[10,22]$ or on the receiver side $[21,23]$, such as a feed forward equalizer (FFE). FFE uses an FIR filter to decrease the gain at low frequencies and increase the gain at high frequencies. The FFE implemented on the transmitter side is called pre-emphasis. Actually it should be called de-emphasis to be accurate. The preemphasis can compensate for the pre-cursor ISI and post-cursor ISI depending on the taps used in the FIR.

The non-linear equalizer cannot be implemented on the transmitter side unless there is an extra connection for feedback [20-22]. The non-linear equalizer implemented on the receiver side using feedback to compensate for the loss in the high frequencies is also called DFE. The DFE can only compensate for the post-cursor ISI but cannot compensate for the pre-cursor ISI [24].

\section{$2.4 \quad$ Feed Forward Equalizer (FFE)}

The discrete time FFE is implemented as an FIR filter. Figure 2.5 shows a typical structure of an FIR filter. 


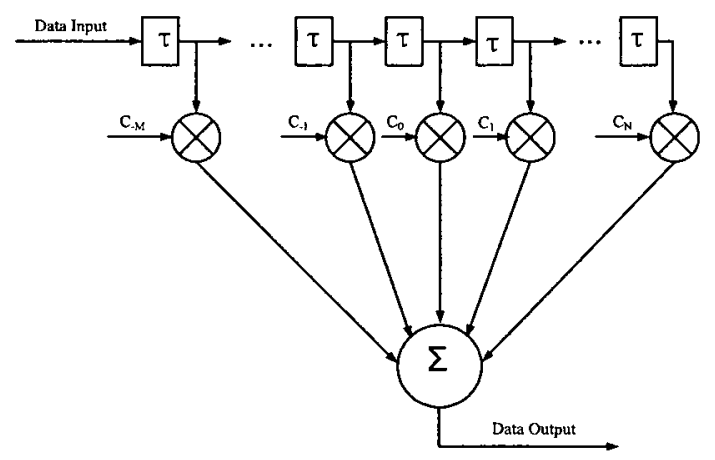

Figure 2.5: Architecture of FIR Filter

In Figure 2.5, $\tau$ is the symbol delay. The number of summed, weighted data sample used is the number of taps of the FIR filter. In Figure 2.5, the FIR filter has $\mathrm{M}$ pre-cursor taps and $\mathrm{N}$ post cursor taps. Including the main tap, the total number of taps of this FIR filter is $(N+M+1)$. If the $\tau$ equals the period of the symbol rate, the FIR filter is called a symbol-spaced FIR filter (SSF). If $\tau$ is less than the period of the symbol rate, the FIR filter is called a fraction-spaced FIR filter (FSF). In the FIR filter, the data is delayed, weighted and added together at the output.

The discrete mathematical expression of an FFE as shown in Figure 2.5 is given in Equation (2.3) $[10,25]$ and the $\mathrm{z}$-domain equation is given in Equation (2.4):

$$
\begin{gathered}
y(n)=\sum_{k=-M}^{N} C_{i} x(n-i) \\
Y(z)=\sum_{k=-M}^{N} C_{i} z^{-i}
\end{gathered}
$$

where $C_{i}$ are the tap coefficients, $\mathrm{N}$ is the number of post-taps, $\mathrm{M}$ is the number of the pre-taps and $\mathrm{z}$ is the delay operator. The total number of taps is $(N+M+1)$. The delay time $\tau$ has the same definition as above and its value depends on tha data rate and whether the FIR filter is SSF or FSF. 
The FFE addresses the ISI by boosting the relative transmit amplitude of highfrequency data or boosting the high-frequency signals at the receiver depending on wether the FFE implemented in the transmitter side or the receiver side. The FFE also boosts the high-frequency noise while it boosts the signals. Another limitation of the FFE is that it cannot reduce or prevent the crosstalk $[16,24]$. Actually, if the FFE is implemented in the transmitter side, the crosstalk becomes worse.

Since the linear equalizer cannot cancel the ISI at both the bit center and bit edge, there are two strategies to optimize the coefficients. One strategy focuses on the bit center and is called Bit-Center NRZ Equalization. Another focuses on the edge of the bit and is called Bit-Edge NRZ Equalizeation.

\subsubsection{Bit-center NRZ Equalizer (BCE-NRZ)}

The BCE-NRZ makes an assumption that the ISI is only important at the sampling point. The classic approach to equalization involves the minimization of the error at the specific sampling point which is at the bit center. The ideal equalized pulse response is $\ldots \begin{array}{llllllll}0 & 0 & 0 & 1 & 0 & 0 & 0 & \ldots\end{array}$ where the 0 's are located at the $\ldots-3,-2,-1,+1$, $+2,+3 \ldots$ bit positions $[1,26]$. The peak of the pulse response is often taken as the cursor [27]. However, this is not the optimal location of the cursor for the eye opening and power consumption for high speed applications or very lossy channels. Optimizing the proper cursor location is the main challenge of the design in these applications. Methods have recently been reported on how to optimize the proper position of cursor $[1,7]$. The location of the cursor is not at the peak of the pulse response, but at a location of a small time prior to the peak. With this method, the jitter can be improved significantly [1].

Once the proper cursor location is determined, the rest of the BCE-NRZ algorithm is based on zero-forcing (ZF) or least mean squared (LMS) error criteria. As there are many books [28] and articles [27] talking about this algorithm, I will not repeat 


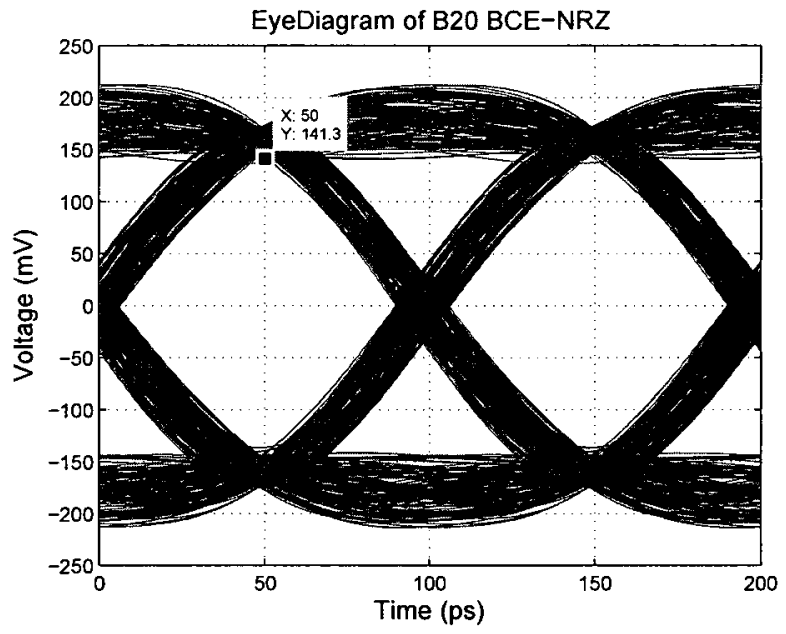

Figure 2.6: Eye Diagram of B20 for $10 \mathrm{Gbps}$ (BCE)

it here. However, the algorithm we used to optimize the coefficients will be discussed in detail in Chapter 3. For the FIR filter with programmable coefficients we can use any applicable algorithm and use the coefficients obtained by other tools and stored in the system. Figure 2.6 shows the eye diagram of the channel B20 used for a 10 Gbps data rate. The height of the eye opening is about $282 \mathrm{mV}$ and the width of the eye opening is about $0.78 \mathrm{UI}$.

\subsubsection{Bit Edge Equalizer (BEE-NRZ)}

The goal of the BEE-NRZ is reduce the ISI at the edge of the bit to zero. That means the response has no ISI at $\pm(n+0.5) T$, where $\mathrm{n}$ is a positive integer [7]. The ideal equalized pulse response is $\ldots \begin{array}{lllllllll}0 & 0 & 0 & 1 & 0 & 0 & 0 & \ldots\end{array}$ where the 0 's are located at the $\ldots-3.5,-2.5,-1.5,+1.5,+2.5,+3.5 \ldots$ bit position [1]. However, they leave a significant amount of ISI at the data centers rendering sampling at these points useless. A strictly BEE-NRZ response has no ISI at $\pm(n+0.5) T$. Therefore, the amplitude at the edges only has 3 levels. Fortunately, if the optimized coefficients would leave some residual ISI at the edges making the eye opening at the data center 


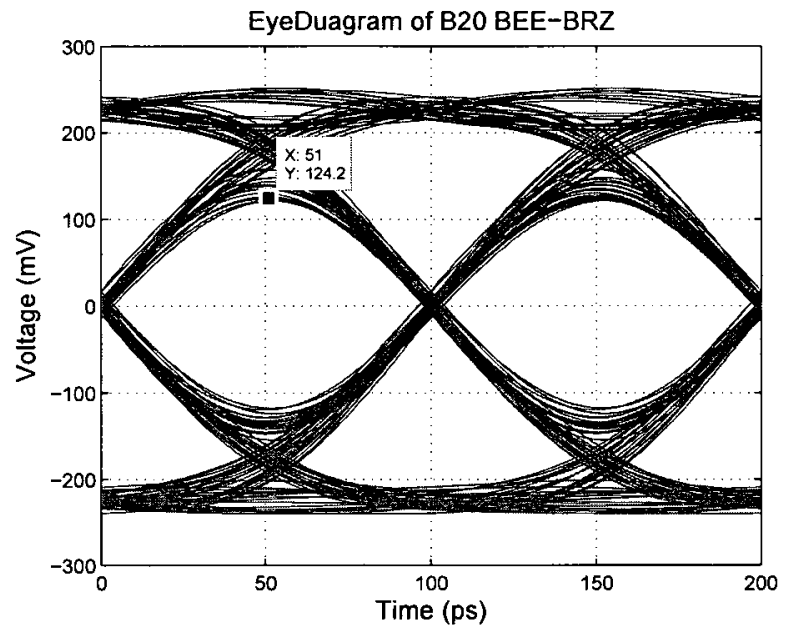

Figure 2.7: Eye Diagram of B20 for $10 \mathrm{Gbps}$ (BEE)

high enough, the detector circuit will work properly. In this case, a 2-level detector circuit can be used. Figure 2.7 shows the eye diagram of the channel B20 for a 10 Gbps data rate. The height of the eye opening is about $248 \mathrm{mV}$ and the width of the eye opening is about $0.89 \mathrm{UI}$.

\subsubsection{Duobinary}

Duobinary signal processing is another way to compress the data spectrum, therefore reduce the required bandwidth for the same data rate compare to the NRZ signals [28]. In the Duobinary algorithm, the uncorrelated 2-level signals are converted into correlated 3-level signals. The duobinary response also has no ISI at $\pm(n+0.5) T$, where $\mathrm{n}$ is a positive integer. From this point view, the duobinary method belongs to the edge equalizing class. Therefore, the amplitude at edges has only 3 levels, so 3 -level signaling sample circuits can be used to detect the amplitude at the edges and then to retrieve data from the correlation between the adjacent symbols. One disadvantage of duobinary signalling is that 3-level signalling leads to more complicated circuits than NRZ signalling in both the transmitter and the receiver. Another disadvantage 


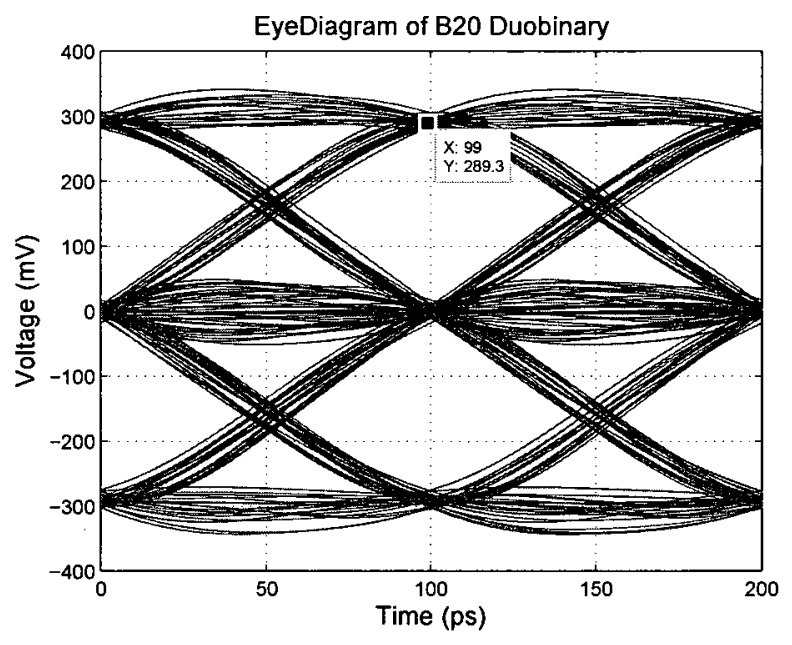

Figure 2.8: Eye Diagram of B20 for 10 Gbps (Duobinary)

is error propagation [28]. As 1-bit datum is retrieved from the two or more received symbols, if one symbol is detected incorrectly, the error can propagate until it is corrected or another error occurs. Furthermore, ternary signalling has worse noise tolerance than binary signalling for a given swing of the waveform.

Recently, in the transmitter, by adding a pre-coder, the 3 levels at the transmitter can be removed, making this transmitter the same as the NRZ transmitter except for the fact that the duobinary equalizer has a pre-coder while the NRZ equalizer does not [26]. On the receiver side, a 3-level slice circuit to sample the received signal is still needed. Figure 2.8 shows the eye diagram of the channel B20 operating at a 10 Gbps data rate. From Figure 2.8, it is clear that the horizontal eye is closed at the bit center, but there are two eyes opened at the bit edge. The vertical openings are $268 \mathrm{mV}$ (upper eye) and $264 \mathrm{mv}$ (lower eye); the horizontal openings are both 0.85 UI. Therefore a 3-level detector circuit can be used to obtain the data.

The lines in the middle of the eye diagram as shown in Figure 2.8 result when the transmitted data are binary 101010, which is preferred for the NRZ equalizer. The popular encoder and decoder systems, such as $8 \mathrm{~B} / 10 \mathrm{~B}$ or $64 \mathrm{~B} / 66 \mathrm{~B}$, are not suitable 
Table 2.2: Comparison of Eye Opening of Different Equalizers

\begin{tabular}{|c|c|c|c|c|}
\hline & \multirow{2}{*}{ BEE } & \multirow{2}{*}{ BCE } & \multicolumn{2}{|c|}{ Duobinary Equalizer } \\
\cline { 4 - 5 } & & & Upper Window & Lower Window \\
\hline Vertical (mV) & 282 & 248 & 268 & 264 \\
\hline Horizontal (UI) & 0.78 & 0.89 & 0.85 & 0.85 \\
\hline
\end{tabular}

for the duobinary equalizer. A new encoder and decoder system must be developed for the duobinary equalizer.

From the above discussion, the amplitude of the received signals of Duobinary is highest due to its bandwidth compression, but it is divided into 2 windows stacked vertically. The BEE-NRZ has the best jitter performance (eye opening width), and BCE-NRZ has the best vertical eye opening. The detailed results of the eye openings for different equalizers are listed in Table 2.2 .

\subsection{Decision Feedback Equalizer (DFE)}

The disadvantage of the FFE is that the noise is also boosted while the FFE boosts the signal at high frequencies. Another equalizer used to compensate for the loss at high frequencies is the DFE implemented at the receiver side. The DFE does not boost high-frequency noise. However, due to the complex algorithm and speed of the circuit, most DFE implementations use only one tap. Although the DFE can remove the ISI of the post cursor, it can not compensate the ISI occurring at the pre-cursor. Therefore, the DFE-only approach is not commonly used. In applications where the channel has both significant pre-cursor and post cursor distortion, the best way is to use the FFE to compensate for the ISI of pre-cursor and to use the DFE to compensate for the ISI at the post cursor [20,29]. 


\subsection{Chapter Summary}

Recent technologies used to solve the ISI due to the limited bandwidth of the channel are discussed in this chapter. The comparisons and results of the different technologies are also discussed. 


\section{Chapter 3}

\section{Pre-Emphasis Design at the System Level}

\subsection{Chapter Overview}

In this chapter, the theory behind the FFE is discussed. The detailed steps of optimizing the coefficients in the frequency domain are given in this chapter. The proposed FIR pre-emphasis architecture is described.

\subsection{Theory of FFE in the Digital Circuit}

As pointed out in Chapter 2, the FFE can be implemented in the transmitter side or the receiver side. As at the receiver side, we do not have the clock information, most FFEs implemented at the receiver side are either an analog FFE $[14,17]$ or a digital FFE using delay line variation between the taps [21]. The difference of the delay values increases jitter. Since the data to be sent are already known and the clock signal is already at the transmitter side, it is easy to implement the FFE in digital circuits, such as in FIR circuits. The delay operation is just the DFF.

Figure 3.1 shows a hybrid analytical model of the transceiver $[7,28]$. Since this Thesis focuses on the equalizer in the transmitter side, the Rx Equalizer in Figure 3.1 can be removed. 


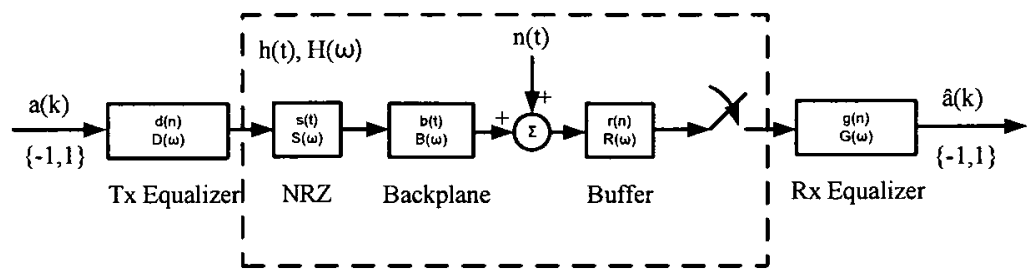

Figure 3.1: An Analytical Model of a Transceiver

The goal in designing a backplane equaliaer is to reduce the ISI. From the time domain and system point of view, a successful transmission means that the received sequence $\hat{a}(\mathrm{k})$ should be a delayed version of the original sequence $\mathrm{a}(\mathrm{k})$. The pulse response can be expressed as Equation (3.1) [7]

$$
h(n T+\phi)=\left\{\begin{array}{ccc}
0 & : & n \neq n_{0} \\
\alpha & : & n=n_{0}
\end{array}\right.
$$

where $h$ is the time domain channel response, $n_{0} T+\phi$ is the delay, and $\alpha$ is the amplitude of the received sequence. The frequency domain response would yield Equation (3.2)

$$
\frac{1}{T} \cdot \sum_{k=-\infty}^{\infty} H\left(\omega-k \omega_{0}\right)=\alpha
$$

In Equation (3.1), a new coordinate is used in which the time origin is at $n_{0} T+\phi$. The reason for this is that if no sample is taken at the time origin, the conversion from a continuous Fourier Transform (CFT) to a discrete Fourier Transform (DFT) will be unnecessarily complicated. In practice, some systems may apply nonlinear operations such as modulus in duobinary (edge equalization) [1] or quantization in decision feedback equalization [20]. In those systems the equivalence between Equation (3.1) and Equation (3.2) does not hold. 


\subsection{Optimizing Coefficients of the FIR Filter}

Since in this Thesis, the goal is to design a digital equalizer in the transmitter side, the design of the equalizer at the system level is to optimize the coefficients of the FIR filter. Optimizing the coefficients can be carried out in the time-domain [27] or the frequency-domain [7]. One of time-domain methods is to sample both the timedomain channel response and the target response, then derive the filter coefficients by deconvolving the channel response and the target response. This method takes only a very limited number of samples of the time-domain channel response. It fails to give optimum results for very challenging channels, such as long delay and high loss channels. Another time-domain method is least mean square (LMS). The LMS method may end up with a large residual error (difficulty of converging) if the first tap is the tap with the maximum coefficient. The reason for this is that it is unable to remove the pre-cursor ISI for the channel with large pre-cursor ISI [7].

Compared to the time-domain method, optimizing coefficients in frequency domain has many advantages. It can optimize the coefficients for any type of channel and it is easy to determine the number of taps needed to compensate for the pre-cursor and post-cursor ISI for a desired residual ISI.

Channel characteristics can be obtained by using a Network Analyzer. The results can be saved in the .sxp file. In the file extension .sxp, x stands for the number of ports. For differential channels $\mathrm{x}$ is 4 . There are several steps to calculate the coefficients of FIR filter in the frequency domain for the channel defined by a .s4p file [7].

The first step is to extrapolate the transfer function from the. $\mathrm{s} 4 \mathrm{p}$ file to the low frequencies and high frequencies according to the data rate of the application and interpolate data between the frequency steps. It is worth noting that in order to get a very fine time resolution of the pulse response, the transfer function needs to be extrapolated to very high frequencies. 
The second step is to get the time-domain pulse response of the backplane channel. The transfer function should be checked to verify whether it is causal or not. If it is not, then the transfer function needs to be modified to be made causal.

The third step is to define the target response of the FIR pre-emphasis NRZ equalizer. As an example, the target response is defined as a raised cosine function as in Equation (3.3).

$$
H_{T}(\Omega)=\left\{\begin{aligned}
0 & : \text { otherwise } \\
1+\cos (\pi \Omega) & :|\Omega| \leq 1
\end{aligned}\right.
$$

where $\Omega$ is the normalized frequency relative to the data rate. Once the circuit topology is finalized, $H_{T}(\Omega)$ should be multiplied with a constant to reflect the maximum output power constraint.

The fourth step is optimizing the time offset of the peak of the channel pulse response to the time origin of the target response. We need it to avoid unnecessary complicated operations in converting the CFT to the DFT. The proper delay also guarantees that the FIR equalizer is causal. This time offset is added to the frequencydomain channel as a positive phase shift.

In the fifth step, the coefficients are derived from the frequency-domain. The DFT of the channel response $\left(H_{D}\right)$ is a folded version of its CFT $\left(H_{C}\right)$ as given in Equation (3.4).

$$
H_{D}(\omega)=\frac{1}{T} \cdot \sum_{k=-\infty}^{\infty} H_{C}\left(\omega-k \omega_{0}\right)
$$

The frequency-domain response of the filter is the division of the target response $H_{T}(\Omega)$ by the folded channel response $H_{D}$ as given in Equation (3.5).

$$
\text { Filter }(\omega)=\frac{H_{T}(\omega)}{H_{D}(\omega)}
$$




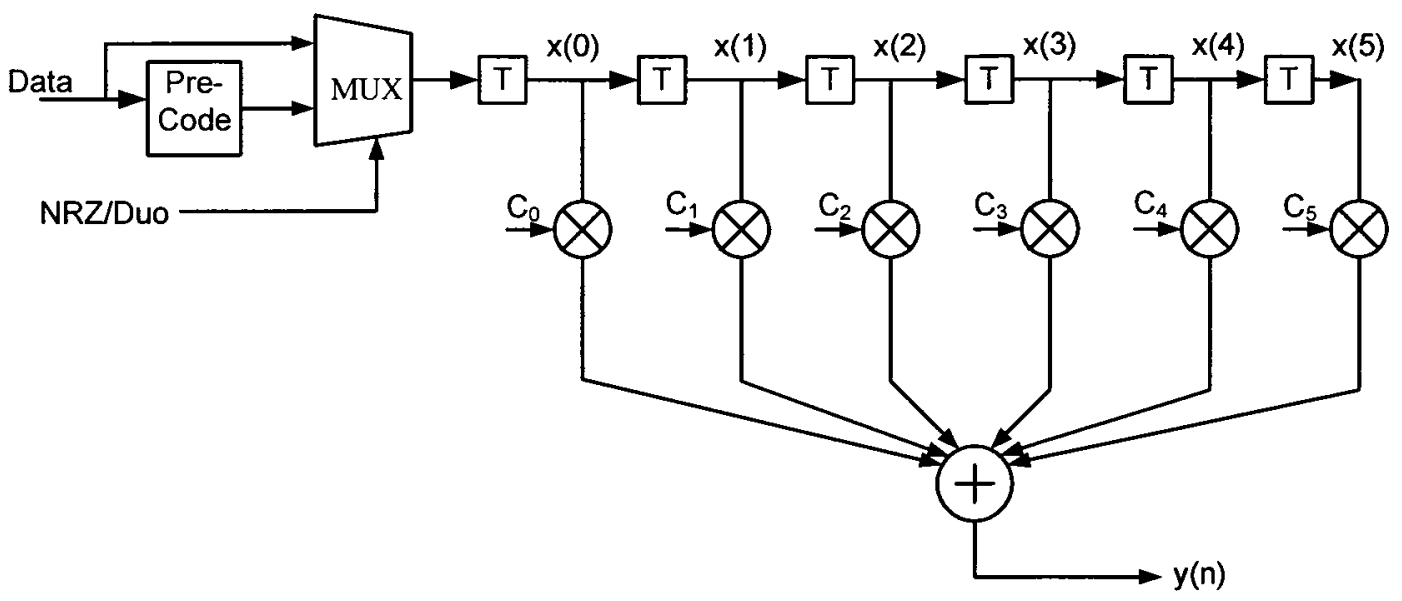

Figure 3.2: Architecture of the Proposed FIR Filter

Usually, the coefficients of the filter is obtained by performing inverse DFT of the frequency-domain filter response, therefore the coefficients should be truncated near the peak coefficient according to the error tolerance.

\subsection{Proposed FIR Pre-emphasis Architecture}

The mathematical expression of a FIR filter is [10,25]:

$$
y(n)=\sum_{i=-M}^{N} C_{i} x(n-i)
$$

where $\left\{C_{i}\right\}$ are the tap coefficients, $M$ is the pre-tap, $N$ is the post-tap, and $C_{0}$ is the main or reference tap. The proposed 6-tap FIR filter is a filter with 5 post-taps which means that $M=0$ and $N=5$ in the Equation (3.6). The optimized coefficients for a specific channel can be acquired from a MATLAB program using a time-domain method which has been developed as described in [27] or a frequency-domain method which has been developed as described in [7]. The architecture of the proposed FIR pre-emphasis block is shown in the Figure 3.2 . 
The proposed equalizer can operate as a BCE-NRZ, BEE-NRZ or Duobinary equalizer depending on the coefficients and Mode selection. The Mode selection NRZ/DUO selects the operation mode of the pre-emphasis circuit. When the NRZ/DUO is low, the pre-emphasis circuit operates in the duobinary with pre-coding mode. In this mode, the input data of the FIR filter is the data after pre-coding. When the NRZ/DUO is high, the pre-emphasis operates in BEE-NRZ or BCE-NRZ mode depending on the coefficients. In this mode, the input of the FIR filter is direct from the input to the block. The data in the FIR filter have one symbol space delay through the data chain. The output is the sum of the current data bit and the previous 5 weighted data bits.

\subsection{Behavioral Model and Verification}

In order to verify the FIR filter, a behavior model was created using the VerilogA

language. The $z$-domain transfer function of the proposed FIR filter is given by Equation (3.7)

$$
Y(z)=\sum_{i=0}^{5} C_{i} z^{-i}=C_{0}+C_{1} z^{-1}+C_{2} z^{-2}+C_{3} z^{-3}+C_{4} z^{-4}+C_{5} z^{-5}
$$

The $z$-domain transfer function of the proposed FIR filter with a pre-coder for the Duobinary equalizer is given by Equation (3.8):

$$
Y(z)=C_{0}+\sum_{i=1}^{5}\left(C_{i-1}+C_{i}\right) z^{-i}+C_{5} z^{-6}
$$

The test bench shown in Figure 3.3 is used to verify the behavioral model of the proposed pre-emphasis circuit.

The optimized coefficients obtained by using the steps discussed in Section 3.3 for 


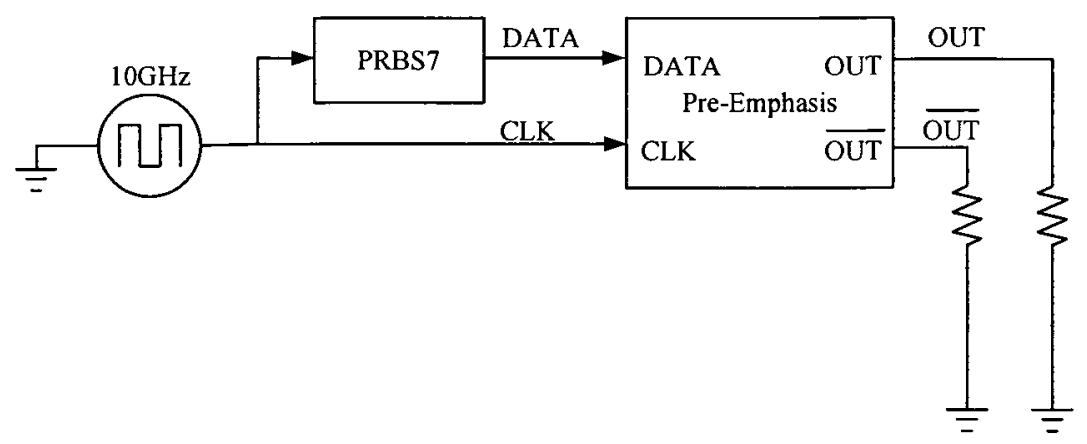

Figure 3.3: Test Bench of FIR Filter (VerilogA Model)

the BCE-NRZ, BEE-NRZ and Duobinary equalizers are listed in the Table 3.1

Figure 3.4 shows the eye diagram of the channel B20 using the VerilogA model and the coefficients are listed in Table 3.1. The heights of the eye opening are 139.06 $\mathrm{mV}$ and $126.89 \mathrm{mV}$ for BCE-NRZ and BEE-NRZ respectively. The horizontal eye opening values are $0.893 \mathrm{UI}$ and $0.868 \mathrm{UI}$ for BCE-NRZ and BEE-NRZ respectively.

From the simulation, the BCE-NRZ is the best choice for the channel B20, it has the largest amplitude signal and widest eye opening (Horizontal). The eye opening values are a little different from the results of Chapter 2 , which were obtained from the simulation results using Matlab. The reason for this is that 10 taps including some pre-cursor and post-cursor taps where used in the Matlab simulation in Chapter 2, but only 5 post-cursor taps were used in the VerilogA model. The eye was closed for the duobinary equalizer, but at the transition edge, we can use a 3-level detector circuit to obtain the data.

\subsection{Chapter Summary}

The detailed algorithm to optimize the coefficients of the FFE are discussed and the behavioral model of the proposed FIR pre-emphasis architecture is created in the VerilogA language. The simulation results of the eye diagram of both the transmitter 
Table 3.1: Coefficients for Channel B20 in Different Models Using VerilogA Blocks

\begin{tabular}{|c|c|c|c|c|c|c|c|}
\hline \multirow{3}{*}{ Model } & & \multicolumn{6}{|c|}{ Coefficients } \\
\cline { 3 - 8 } & & $\mathbf{0}$ & $\mathbf{1}$ & $\mathbf{2}$ & $\mathbf{3}$ & $\mathbf{4}$ & $\mathbf{5}$ \\
\hline \multirow{3}{*}{ NRZ-BCE } & Coeff & 1 & -0.5953 & 0.1053 & -0.0113 & -0.0394 & 0.014 \\
\cline { 2 - 8 } & Current $^{1}(\mathrm{~mA})$ & 11.3295 & -6.7445 & 1.1930 & -0.1280 & -0.4464 & 0.1586 \\
\cline { 2 - 8 } & Digital Coeff $^{2}$ & 23 & -13 & 2 & 0 & -1 & 0 \\
\hline \multirow{3}{*}{ NRZ-BEE } & Coeff & 1 & -0.4974 & 0.0284 & 0.0084 & -0.0718 & 0.0506 \\
\cline { 2 - 8 } & Current $^{1}(\mathrm{~mA})$ & 12.0729 & -6.0051 & 0.3429 & 0.1014 & -0.8668 & 0.6109 \\
\cline { 2 - 8 } & Digital Coeff $^{2}$ & 24 & -12 & 1 & 0 & -2 & 1 \\
\hline \multirow{3}{*}{ Duobinary } & Coeff & 1 & 0.4033 & -0.5560 & 0.1256 & -0.0660 & -0.0258 \\
\cline { 2 - 8 } & Current $^{1}(\mathrm{~mA})$ & 9.1882 & 3.7056 & -5.1087 & 1.1540 & -0.6064 & -0.2371 \\
\cline { 2 - 8 } & Digital Coeff $^{2}$ & 18 & 7 & -10 & 2 & -1 & 0 \\
\hline
\end{tabular}

Note:

1: The total absolute current of all taps is $20 \mathrm{~mA}$.

2: 1 LSB is equal to $0.5 \mathrm{~mA}$.

and receiver side shows improvement of the eye opening using the proposed preemphasis circuit. 


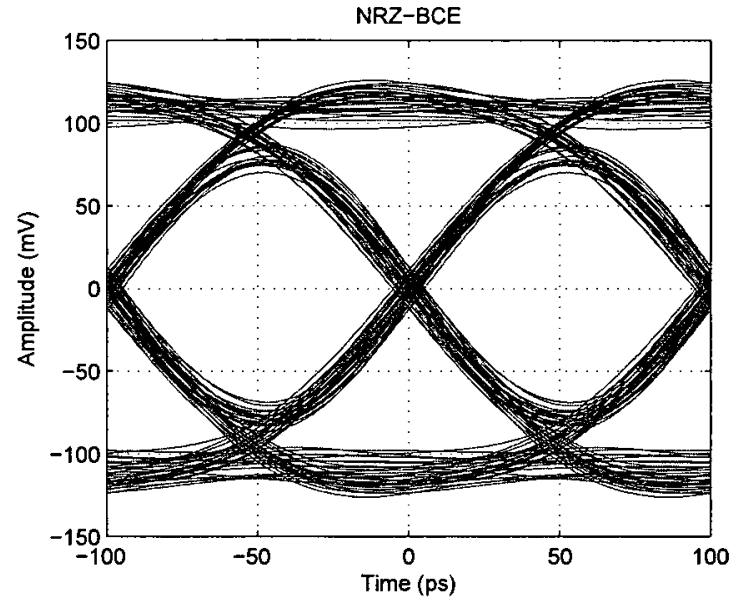

(a) NRZ-BCE

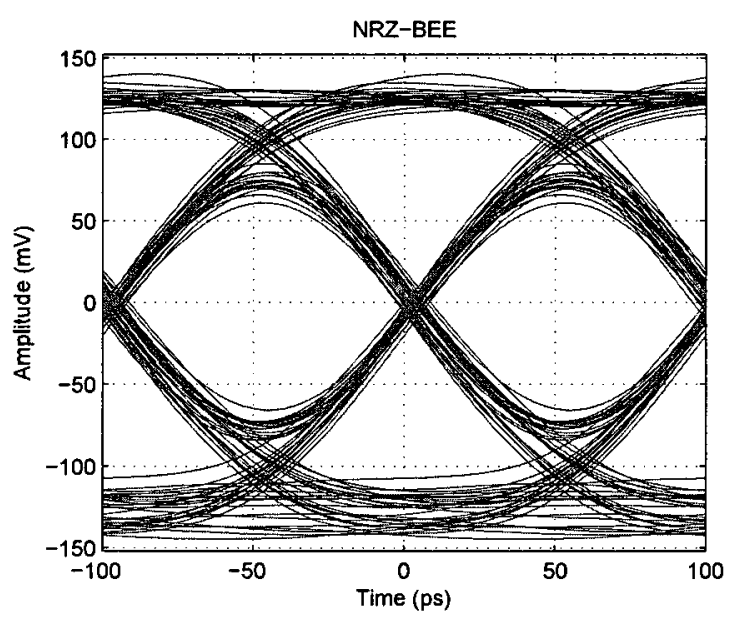

(b) NRZ-BEE

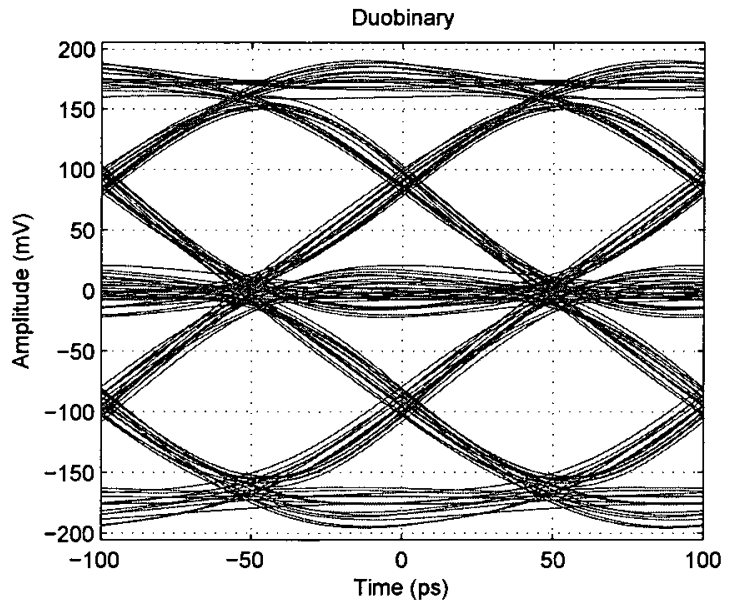

(c) Duobinary

Figure 3.4: Eye Diagram of B20 for Differential Equalizers Using VerilogA Model 


\section{Chapter 4}

\section{Design of the FIR Pre-emphasis Circuit in 90nm CMOS Technology}

\subsection{Chapter Overview}

The detail circuits and layout of the proposed pre-emphasis circuit in a $90 \mathrm{~nm}$ CMOS technology are discussed in this chapter. The results of the schematic simulation and post layout simulation are given.

\subsection{FIR Pre-emphasis Circuit Block Diagram}

Figure 4.1 shows the FIR pre-emphasis circuit block diagram [10]. The circuits consist of a Single-end-Signal-to-Differential-Signal-Converter (STDC), a Pre-code circuit, a MUX circuit, a Re-timing circuit, a Pre-driver with coefficient sign control, and a preemphasis driver with coefficient control. The STDC converts the single ended signal to a differential signal. The Pre-code circuit consists of a DFF and an XOR, which is used for the duobinary equalizer. The pre-code and MUX circuits select the equalizer operation mode. When the equalizer operates in the BCE-NRZ mode or BEE-NRZ mode, the MUX selects the data directly from the output of the STDC of "Data In" 


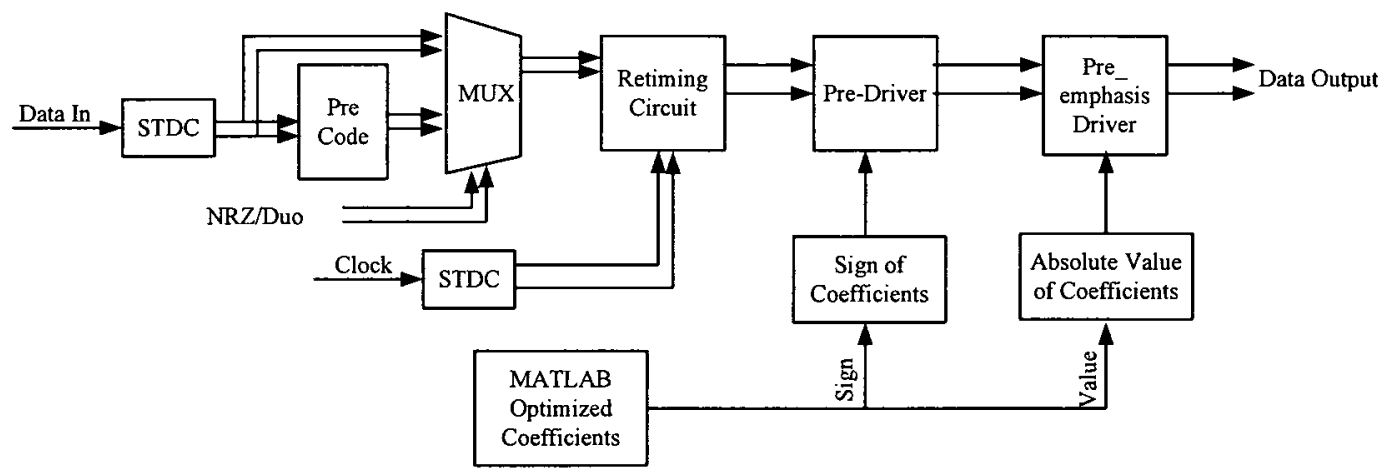

Figure 4.1: Proposed FIR Pre-emphasis Circuit Block Diagram

and the pre-code circuit is bypassed. When the equalizer operates in the Duobinary mode, the MUX selects the data from the output of the pre-code circuit. The retiming circuit consists of the DFFs connected in series to provide six data samples used in the FIR filter. Each data occupies one symbol space. The sign of coefficients is controlled through the XOR gate in the pre-diver. The pre-driver consists of buffers to drive the large CMOS transistors in the pre-emphasis driver circuit. The absolute value of the coefficients controls the weight of each tap by controlling the tail current of the pre-emphasis driver.

The DFFs, XORs, MUX and buffer can be implemented in either rail-to-rail CMOS circuits or in Current Mode Logic (CML) circuits. At low frequencies, CMOS rail-to-rail is preferred for its simplicity and low static power dissipation while at

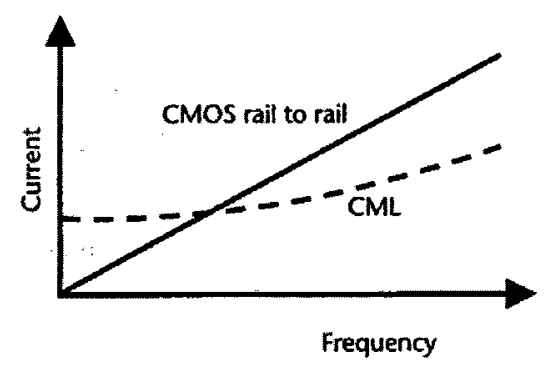

Figure 4.2: Comparison of Current for CMOS Rail-to-Rail and CML circuit Vs Frequency 


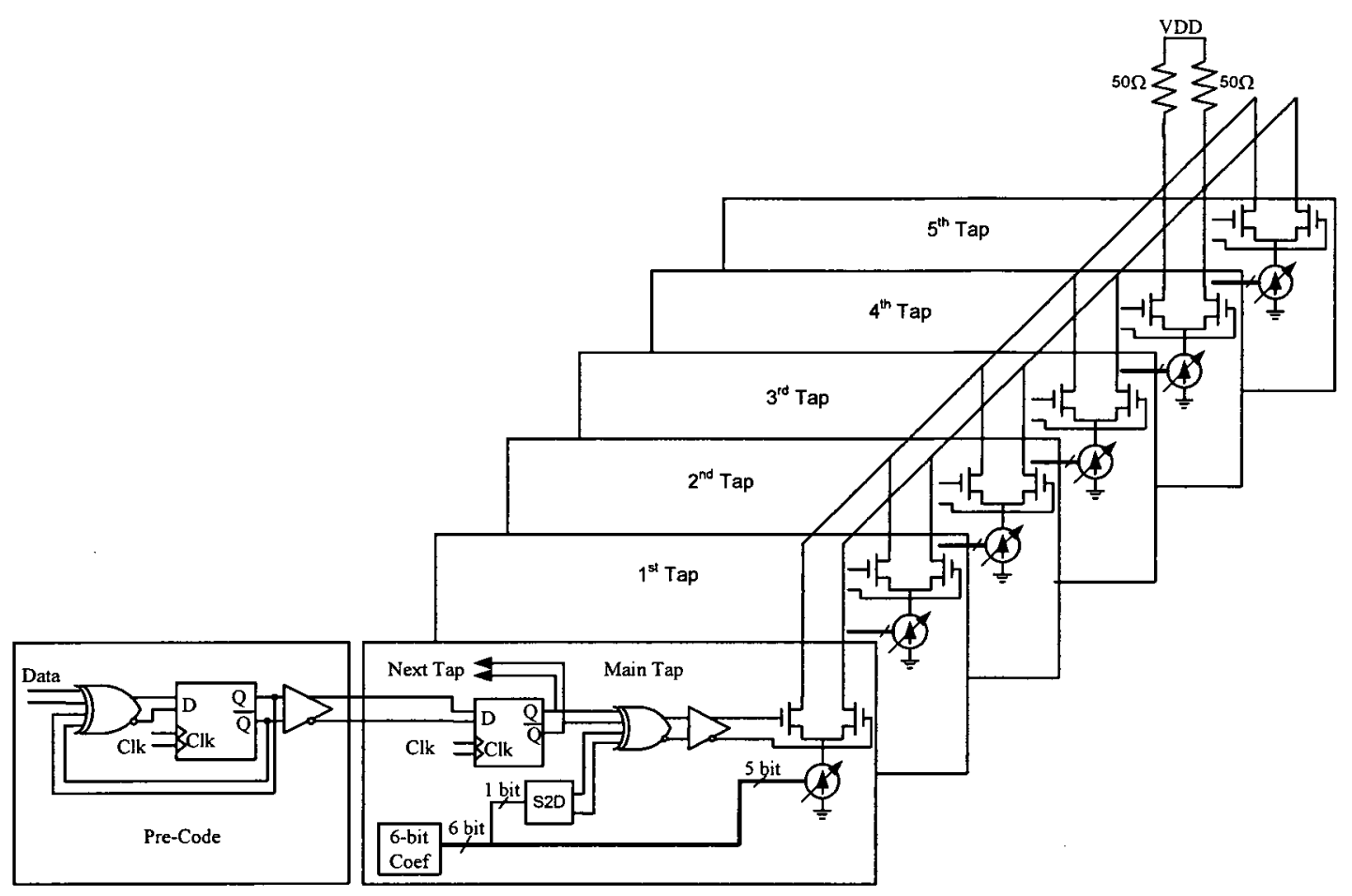

Figure 4.3: Top Level of the 6-Tap FIR Filter

high frequencies, CML is used, as it can operate faster with lower power because of the reduced output swing. When there is no transition the CMOS rail-to-rail circuit does not consume any current (except the leakage current, which is very small), while CML does. The comparison of current versus frequency between the rail-to-rail CMOS circuit and the CML circuit is shown in Figure 4.2. Although in the sub-micro technologies, such as $90 \mathrm{~nm}$ and below, the leakage current is larger compared to the large geometry technologies such as $.18 \mu \mathrm{m}$ or $.25 \mu \mathrm{m}$, rail-to-rail CMOS circuits consume most power during the transitions, and its power consumption is proportional to the operational frequencies. Just like the rail-to-rail CMOS circuit does, the CML bias current must rise, therefore the power consumption rises as the speed of switching increases but the power rises at a lower rate [30]. Another advantage of using the CML is that it is differential and has a good power-supply rejection which is preferred 
in many high speed and lower output swing circuits.

Figure 4.3 shows the top level blocked diagram of the 6-tap programmable FIR pre-emphasis circuit [10]. The STDC circuits for the input data and clock are not included in Figure 4.3. Given that the coefficients (loaded when the chip is initialized) do not change when operated for a specific channel, register circuits that store the coefficients and STDC for sign control are implemented as rail-to-rail static CMOS circuits to reduce the power for the reason discussed above. In this work, as the coefficients do not change after initialization, the power for STDC circuits for the sign control is very low, only the leakage current, which is very small. All other circuits in Figure 4.3 are implemented using CML circuits. Each tap consists of a DFF for retiming, an XOR for controlling the coefficient sign, buffers to provide driving ability to the pre-emphasis driver, a Digital-to-Analog Converter current source (iDAC) to control the weight of each tap, and a register to store the optimized coefficient value (including the sign and absolute value of the coefficients). The sign of the coefficient is stored in the Most Significant Bit (MSB). To simplify the digital circuits for the coefficients, sign magnitude binary code is chosen for the coefficients. As selection of these codes and the controlled current relationship are listed in Table 4.1. The coefficient values can be programmed through JTAG or simple shift registers.

Table 4.1: Representative Sample of 6-Bit Signed Digital Code and Current Relationship

\begin{tabular}{|c|c|c|c|c|c|c|c|}
\hline Code & 011111 & 010000 & 001000 & 000100 & 000010 & 000001 & 000000 \\
\hline Number & +31 & +16 & $+8^{`}$ & +4 & +2 & +1 & +0 \\
\hline Current(mA) & +15.5 & +8 & +4 & +2 & +1 & +0.5 & +0 \\
\hline Code & 111111 & 110000 & 101000 & 100100 & 100010 & 100001 & 100000 \\
\hline Number & -31 & -16 & $-8^{\star}$ & -4 & -2 & -1 & -0 \\
\hline Current(mA) & -15.5 & -8 & -4 & -2 & -1 & -0.5 & -0 \\
\hline
\end{tabular}

In order to save power, all DFFs and XORs are optimized for power consumption 
according to their loads. For example, the loads for the DFF are one DFF and one XOR therefore we do not need to design a high fan out DFF, such as fan out 4 . A large fan out means a heavy load and the circuit requires more power. For the same reason, the XOR is designed only to drive one buffer.

\subsection{Current Mirror (CM)}

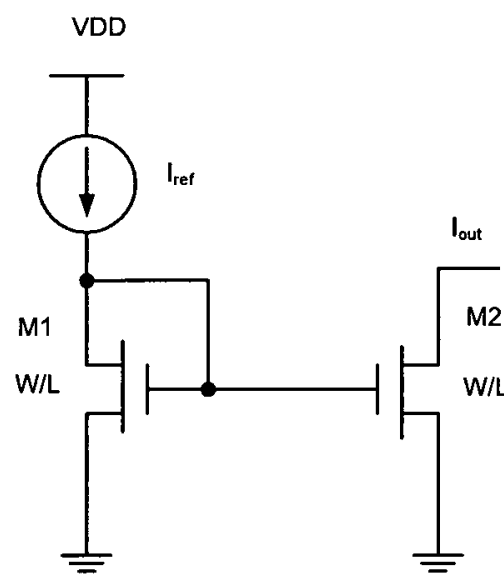

(a) Simple Current Mirror

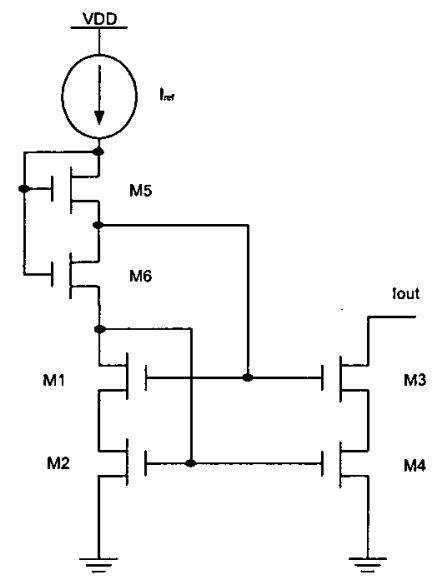

(c) Sooch Current Mirror

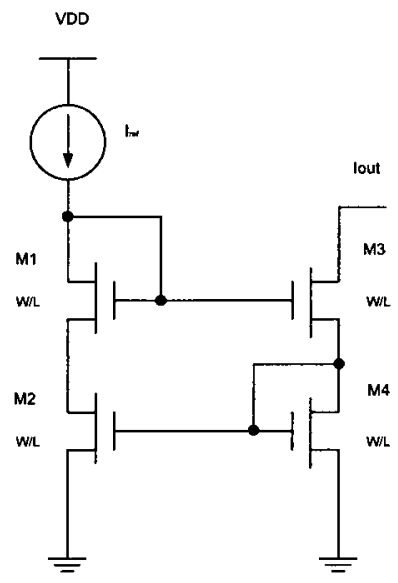

(b) Wilson Current Mirror

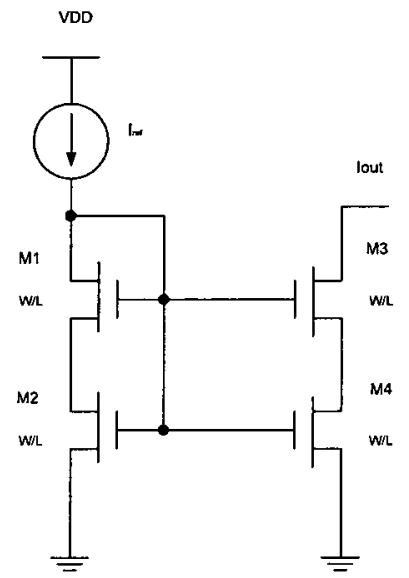

(d) Stacked Current Mirror

Figure 4.4: Different Current Mirror Circuits

All CML circuits have the tail current source. In order Reduce power and chip area 
and to minimize the relative error between the current sources, all current sources of the CML circuits are mirrored from the same current mirror. There are many types of current sources. Figure 4.4 shows the circuits of (a) the simple current mirror, (b) the Wilson current mirror, (c) the Sooch current mirror [31] and (d) stacked current mirror [10].

The output current is only determined by the physical ratio of the transistors and the input reference current. For example, the output of the current of the simple current mirror is given in Equation (4.1) [31]:

$$
I_{O U T}=\frac{(W / L)_{2}}{(W / L)_{1}} I_{r e f}
$$

The output resistance, minimum output voltage and systematic gain error of the simple current shown in Figure 4.4 (a) are given in Equations (4.2), (4.3) and (4.4):

$$
\begin{gathered}
R_{O U T}=\frac{1}{\lambda I_{D 2}} \\
V_{O U T(\text { min })}=V_{o v}=\sqrt{\frac{2 I_{O U T}}{\mu C_{O X}(W / L)_{2}}} \\
\epsilon=\frac{V_{D S 2}-V_{D S 1}}{V_{A}}
\end{gathered}
$$

Here $V_{A}$ is the Early Voltage.

The output resistance, minimum output voltage and systematic gain error of the Wilson Current Mirror shown in Figure 4.4 (b) are given in Equation (4.5), (4.6) and (4.7):

$$
\begin{gathered}
R_{O U T}=\frac{1}{g_{m 4}}+r_{o 3}+g_{m 3} r_{o 3}\left(1+\frac{1}{g_{m 4} r_{o 3}} r_{o 2} \approx\left(1+g_{m 3} r_{o 2}\right) r_{o 3}\right. \\
V_{O U T(m i n)}=V_{t h}+2 V_{o v} \\
\epsilon=\frac{V_{D S 4}-V_{D S 2}}{V_{A}}=-\frac{V_{G S 3}}{V_{A}}
\end{gathered}
$$


Considering the body effect of the transistor M3, Equation (4.5) should be modified to the following equation:

$$
R_{O U T} \approx\left(2+g_{m 3} r_{o 2}\right) r_{o 3}
$$

The output resistance, minimum output voltage and systematic gain error of the Sooch Current Mirror shown in the Figure 4.4 (c) are given in Equations (4.9), (4.10) and (4.11):

$$
\begin{gathered}
R_{O U T}=r_{o 3}\left[1+\left(g_{m 2}+g_{m b 2}\right) r_{o 1}\right]+r_{o 1} \\
V_{O U T(m i n)}=2 V_{o v} \\
\epsilon=\frac{V_{D S 4}-V_{D S 2}}{V_{A}}=\frac{V_{o v 1}-\left(V_{t h}-V_{o v 1}\right)}{V_{A}}=-\frac{V_{t h}}{V_{A}}
\end{gathered}
$$

If $V_{D S 4}$ equals to $V_{D S 2}$, then there is no error. The transistor $M_{1}$ operates in the saturation region if $V_{t h}>V_{o v}$. Although this condition is usually satisfied, a low threshold and/or high override voltage may cause $M_{1}$ to operate in the triode region. If this happens, $V_{G S 4}$ depends strongly on $V_{D S 4}$, increasing the systematic gain error. Therefore, checking the region of operation of $M_{4}$ at all corners of the PVT is very important in the design of the Sooch current mirror. Another limitation of the high-swing cascode current mirror is that the input voltage is very large [31].

Two transistors with the same width connected in series can be replaced by one transistor with the same width but the length is the sum of the two lengths [10]. The performance analysis is the same as the simple current mirror.

Figure 4.5 shows the performance of these current mirrors. From Figure 4.5 and from the above equations, we can see that the Wilson current mirror has the best performance. In our design, when $V_{d s}>0.4 \mathrm{~V}$, the current $I_{d s}$ almost does not change, but it needs high minimum output voltage. When $V_{d s}<0.34 \mathrm{~V}$, the current $I_{d s}$ decreases quickly. The minimum output voltage of the other three current mirror 


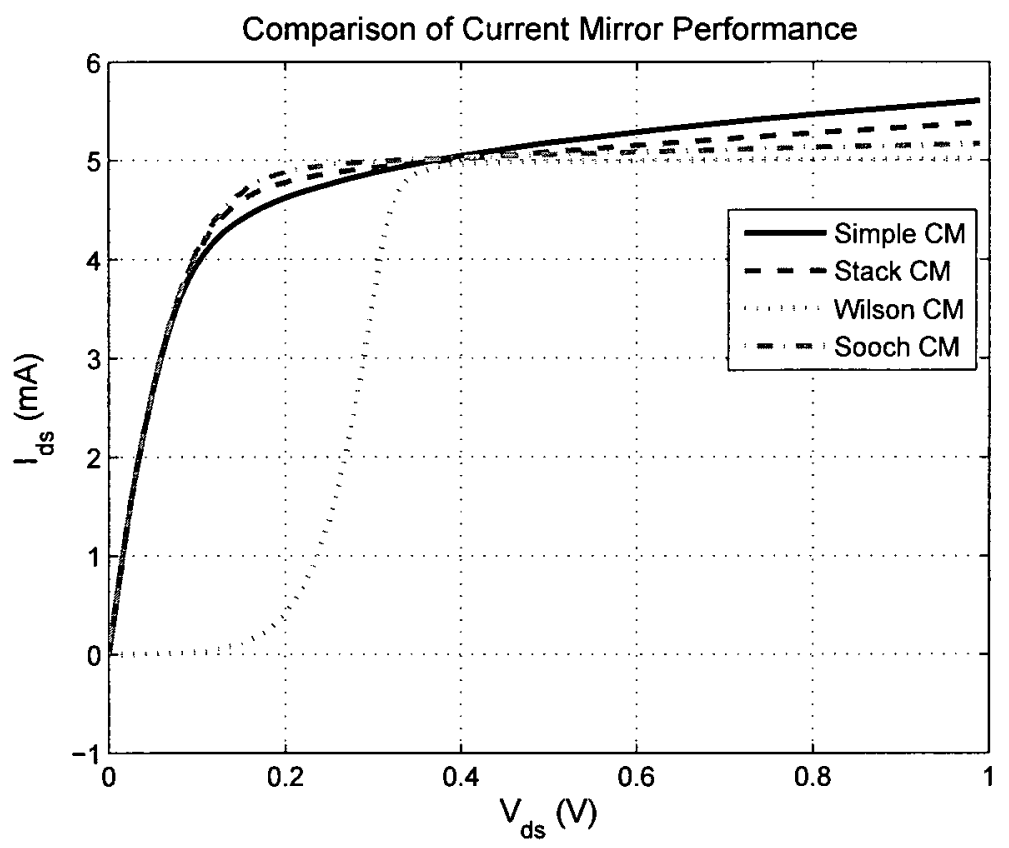

Figure 4.5: Comparison Performance of Different Types of Current Mirror

is in the same range. In these three current mirrors, the Sooch current mirror has the best performance and simple current mirror has the worst performance. The voltage of the $M_{5}$ drain terminal in the Sooch CM is high, that makes more challenge to design Sooch CM for lower power supply circuit. Although there is an improved Sooch current mirror which separates the four transistors in the input side to two branches with two transistors in each branch, the improved circuit consumes more power and requires more chip area compared to the other three CMs. Based on these reasons, the stacked CM with low $V_{t h}$ on the top and standard $V_{t h}$ on the bottom is employed in the proposed circuit.

In this design, the stacked current mirror is chosen based on the following reasons. First, it is simple and has low minimum output voltage. Second, the performance is good enough for the design. We can replace the stacked transistors with a longer transistor, however, we can also use different threshold voltage transistors to optimize the design if the stacked CM circuits are used, since in the utilized 90nm technologies, 
there are three different threshold voltage transistors, namely high threshold, standard threshold and low threshold voltage.

\subsection{DFF, XOR, Buffer and Mux}

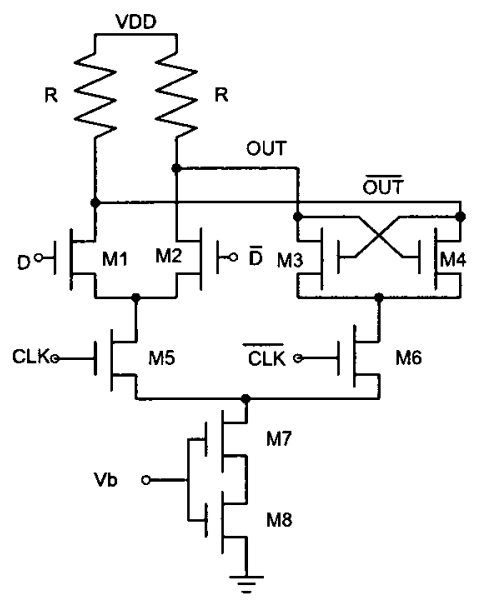

(a) D-Latch

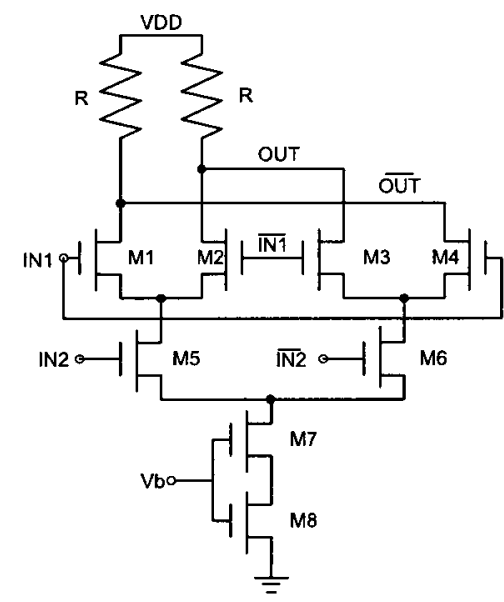

(b) XOR

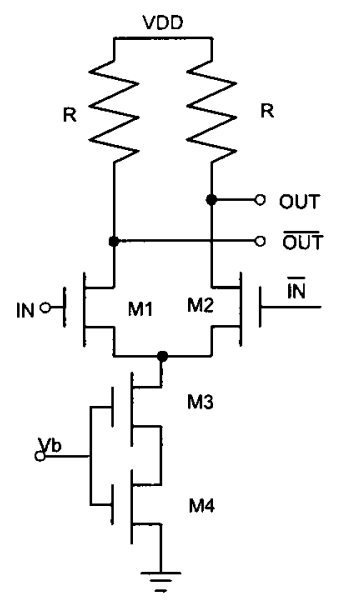

(c) Buffer

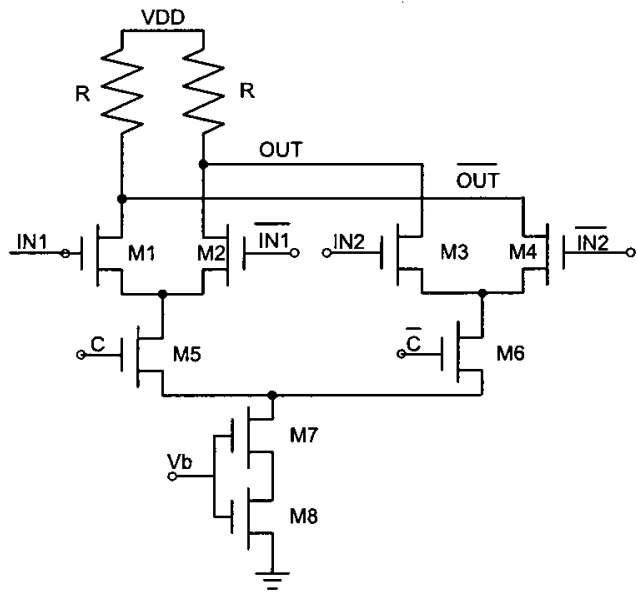

(d) MUX

Figure 4.6: D-Latch, XOR, Buffer and MUX in CML

The DFF consists of two D-Latches with a Master-Slave Structure. The transistor level circuits of the D-Latch, XOR, Buffer and Mux are shown in Figure 4.6. When 
the CMOS operates in saturation, the current is defined by Equation (4.12) [30]:

$$
I_{D}=\frac{1}{2} \mu C_{o x} \frac{W}{L}\left(V_{O D}^{2}\right)
$$

In order to reduce the size of the transistors and therefore reduce the input capacitance of the transistors, we select the minimum length for all transistors except the transistors used for the current mirror. All transistors in the circuit are transistors with low $V_{t h}$. From Equation (4.12), we can see that for the same transistor size, the current is the ratio of square of the over-drive voltage, which is defined by $V_{O D}=V_{G S}-V_{t h}$. If we use low threshold voltage $\left(V_{t h}\right)$ transistors, the circuit can sense a smaller $V_{G S}$ as compared to the regular $V_{t h}$ transistors and thus, a pair of transistors in CML can fully switch more easily and faster at the cost of a larger leakage current. In general, with a CML circuit, more current results in faster operation. This is a basic tradeoff between current, load resistance and capacitance. Although we can not change the load resistance at the Pre-Emphasis driver, which has to match the channel impedance of $50 \Omega$, we can trade off current and load resistors for the D-latches, XORs and Buffers. For a given desired output swing $V_{O}$, the required transistor size and current is given by Equation (4.13) [30]:

$$
V_{O}=\sqrt{\frac{2 I_{E E}}{\mu C_{o x}(W / L)}}
$$

here $I_{E E}$ is the tail current of the CML circuit.

If we lower the output swing for a given current, we can use smaller sized transistors to reduce the capacitance and improve the speed of the circuit. On the other hand, using a small output swing means that the next stage has to sense lower input voltage. This means that larger sized transistors are required for the next stage. In our design, the output swing is $0.4 \mathrm{~V}$ and the current of the D-latch, Buffer and XOR is $0.5 \mathrm{~mA}$. 
Since the CML circuit is a differential circuit, the common mode voltage also has to be set to an appropriate value, otherwise, the circuit may not work. In our design, the common mode voltage chosen is $0.8 \mathrm{~V}$ for the DFFs, XORs, and pre-emphasis driver and buffers that drive the pre-emphasis driver. The power supply for this design is $1.0 \mathrm{~V}$.

\subsection{Pre-emphasis Driver Circuit}

\subsubsection{Sizing Transistors in the Output Stage}

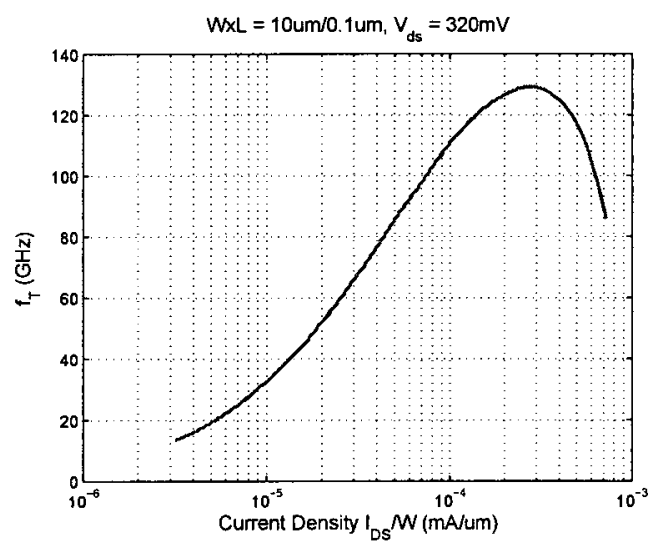

(a) $f_{T}$ Vs Current Density

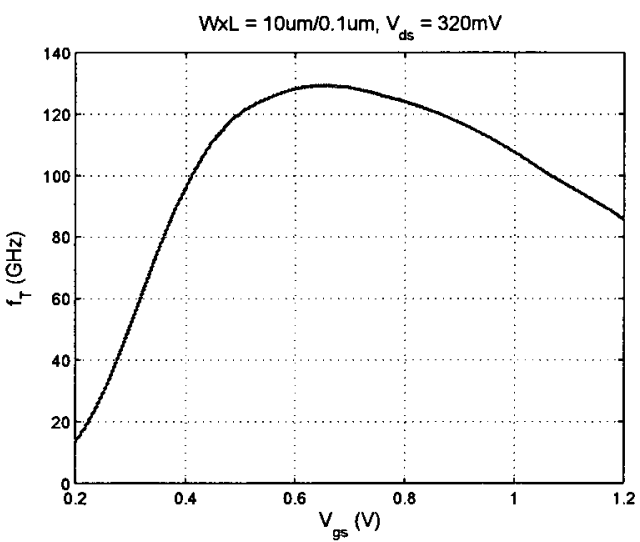

(b) $f_{T}$ Vs $V_{g s}$

Figure 4.7: $f_{T}$ vs Current Density and $V_{g s}$ (NMOS W $\times \mathrm{L}=10 \mu \mathrm{m} \times 0.1 \mu \mathrm{m}, 90 \mathrm{~nm}$ Technology)

In high speed circuit design, if the CMOS transistors are biased at the maximum $f_{T}$, this gives a current density of around $0.28 \mathrm{~mA} / \mu \mathrm{m}[32]$. Figure 4.7 shows the simulation results of $f_{T}$ versus current density and $V_{g s}$ for a $10 \mu \mathrm{m} \times 1 \mu \mathrm{m}$ NMOS transistor in $90 \mathrm{~nm}$ technology. The pre-emphasis driver consists of several pairs of NMOS transistors and several iADC circuits depending on number of the taps. Figure 4.8 (a) shows the traditional one tap of the FIR pre-emphasis driver with a 5-bit digital controlled current source. The challenge choosing the size of the transistors for a wide 


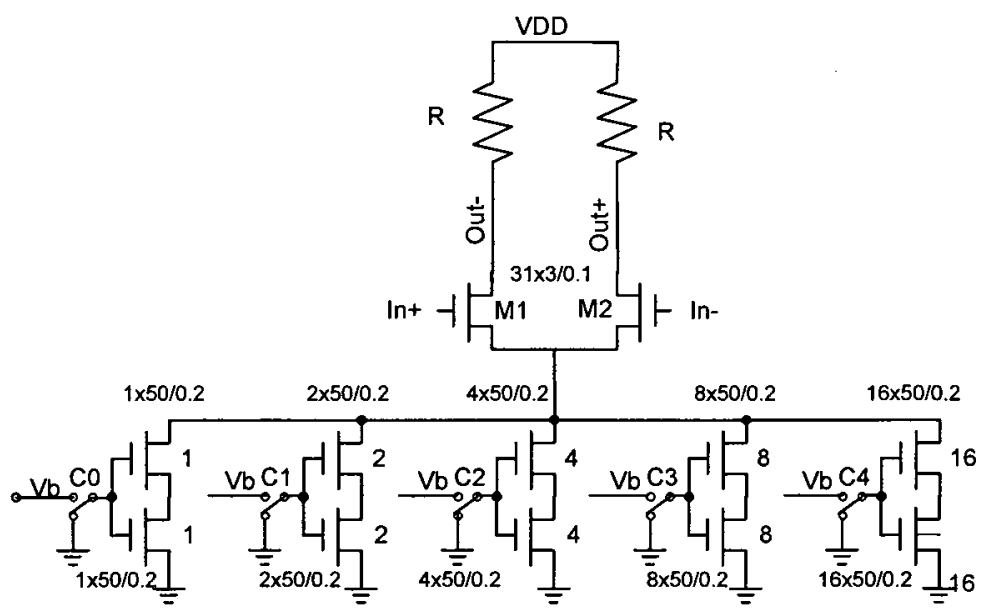

(a) Traditional Circuit

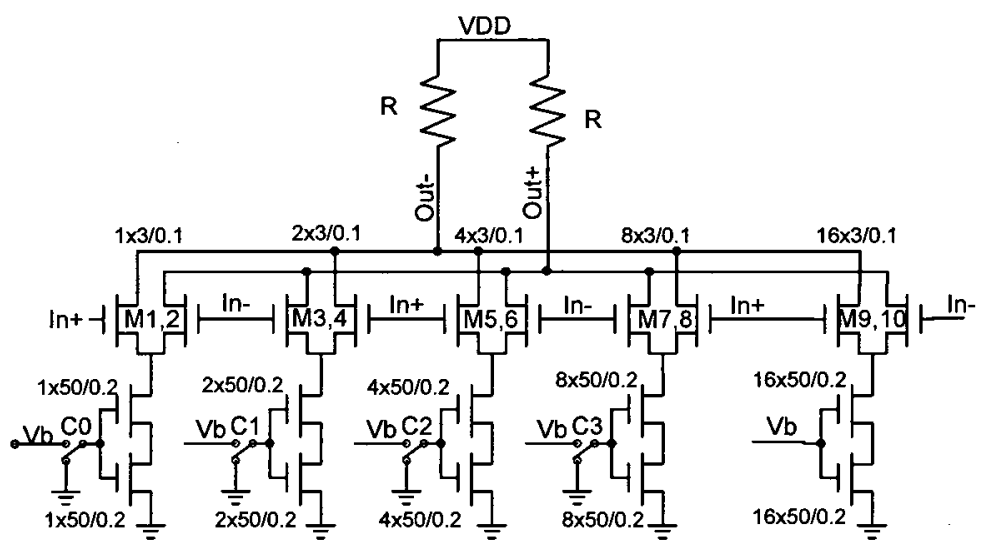

(b) Proposed Circuit

Figure 4.8: One Tap Pre-emphasis Driver

range of current change. For example, if the maximum current for the specific tap is $10 \mathrm{~mA}$ for a specific channel, but for another channel the maximum current is 1 $\mathrm{mA}$, if we choose the size of the transistor to meet the maximum $f_{T}$ according to the maximum possible current, this gives us the width of the transistor as $35.7 \mu \mathrm{m}$ and $f_{T}$ as $129 \mathrm{GHz}$. When the circuit operates for another channel, the current is $1 \mathrm{~mA}$ and the current density is $0.028 \mathrm{~mA} / \mu \mathrm{m}$, and the resulting $f_{T}$ is $62.6 \mathrm{GHz}$. If the current change is wider, the $f_{T}$ drops more. For example, in this design, for a 5-bit iDAC and a current of $0.5 \mathrm{~mA}$ is used for $1 \mathrm{LSB}$. The maximum current is $15.5 \mathrm{~mA}$ 


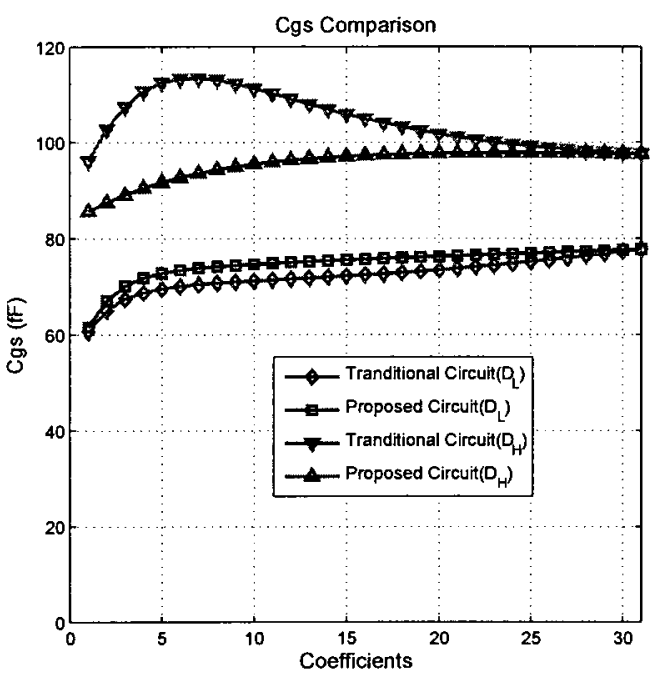

(a) $C_{g s}$

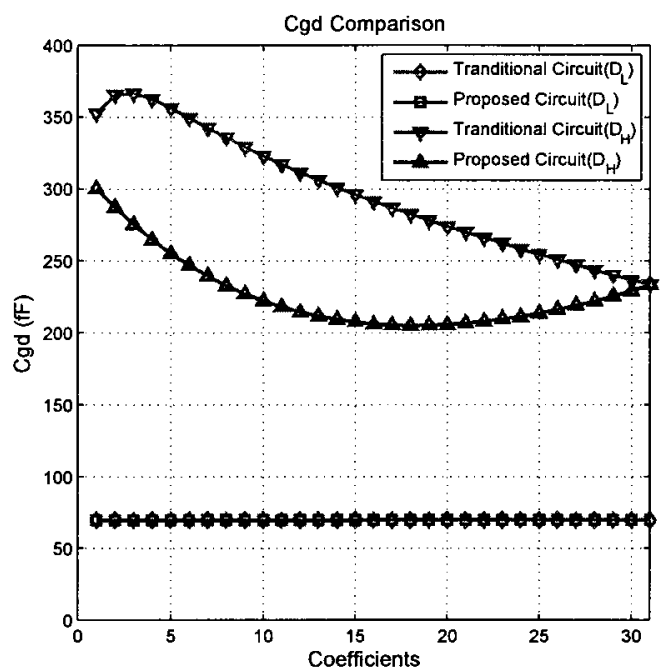

(b) $C_{g d}$

Figure 4.9: $C_{g s}$ and $C_{g d}$ vs Coefficients

and the minimum current is $0.5 \mathrm{~mA}$. The ratio is $31(15.5 \mathrm{~mA} / 0.5 \mathrm{~mA})$. In this case, the $f_{T}$ drops to $30 \mathrm{GHz}$ when the current is only $0.5 \mathrm{~mA}$ if we use the width to get the maximum $f_{T}$ for the maximum current. Furthermore the actual $f_{T}$ of the transistors changes according to the gate-source voltage $\left(V_{g s}\right)$ and the drain-source voltage $\left(V_{d s}\right)$. A reasonable $V_{g s}$ and $V_{d s}$ may be just $0.3 \mathrm{~V}$ to $0.6 \mathrm{~V}$ because the transistors are stacked vertically and the maximum power supply voltage is limited. That results in lowering the $f_{T}$ of the transistors further and limiting the speed of the circuit.

The proposed one tap pre-emphasis driver shown in the Figure 4.8 (b) meets the requirement of this challenge. In this circuit, the current source is controlled by a 5-bit digital signal. The current of each branch is fixed when the control bit is high or the current is zero when the control bit is low. The transistors M1 to M10 can be sized to make the current density the same for all current branches when the control bit is high. There are several benefits for using the proposed circuit.

One benefit of using the proposed circuits is in reducing the parasitic capacitance $\left(C_{g s}\right.$ and $\left.C_{g d}\right)$ of the pair of transistors. Figure 4.9 shows the $C_{g s}$ and $C_{g d}$ (M1 in 
Table 4.2: Traditional Circuit and Proposed Circuit Capacitance Comparison

\begin{tabular}{|c|c|c|c|}
\hline & Data Input & Traditional Circuit & Proposed Circuit \\
\hline \multirow{3}{*}{$C_{g s}(\mathrm{fF})$} & $\mathbf{L}$ & 156 & 158 \\
\cline { 2 - 4 } & $\mathbf{H}$ & 207 & 195 \\
\hline \multirow{3}{*}{$C_{g d}(\mathrm{fF})$} & $\mathrm{L}$ & 144 & 144 \\
\cline { 2 - 4 } & $\mathbf{H}$ & 619 & $\mathbf{5 0 6}$ \\
\hline
\end{tabular}

Figure 4.8 (a) or M1,4,5,8,9 in Figure 4.8 (b)) changing when the 5-bit coefficients change from 1 to $31[10]$. When the input is high, $C_{g s}$ and $C_{g d}$ of the traditional circuit is larger than those of the proposed circuit (Figure 4.9 (a) and (b)). When the input is low, the $C_{g s}$ and $C_{g d}$ of both circuits are similar (Figure 4.9 (a) and (b)). Table 4.2 compares of the capacitance of both circuits using the coefficients used for simulating the NRZ-BCE (the coefficients are 23, $-13,2,0,-1,0$ ). Comparing the traditional circuits and proposed circuits, $C_{g s}$ is reduced from $207 \mathrm{fF}$ to $195 \mathrm{fF}$, a drop of $5.8 \%$ when the transistors are switched on. On the other hand, $C_{g d}$ reduces from $619 \mathrm{fF}$ to $506 \mathrm{fF}$, a drop of $18.3 \%$ when the transistors are switched on. Reducing the input capacitance means the transistors of the pre-emphasis driver can turn on and off more quickly. Therefore, the circuits can have a better performance using the same power consumption or use less power to achieve the same performance.

Another benefit of using the proposed circuits is that the performance current source is improved due to the reduced input and output capacitance and therefore less charge sharing. Figure 4.10 shows the comparison of the current error and variance of the two circuits. From 4.10, it is clear that the performance of the current source of the proposed circuit is much better than the traditional circuit. The proposed circuit has less relative output error and ripples of the current source. Figure 4.11 shows the output of the current source of both circuits at different coefficients (9 and 15) using the 0101 data pattern. From the Figure 4.11, we can see that there is not much 


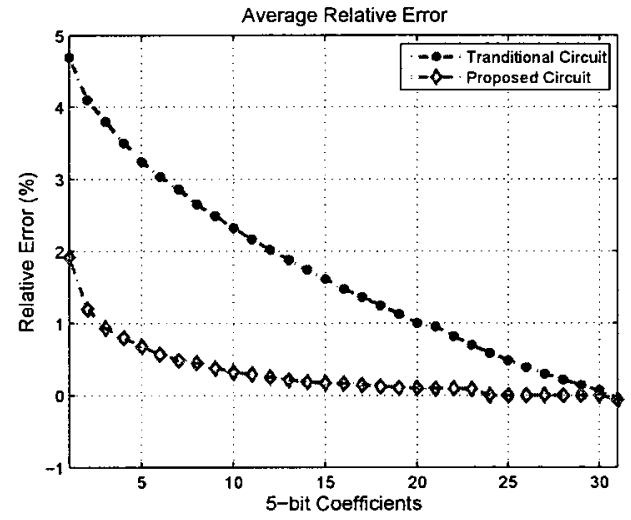

(a) Coeff $=9$

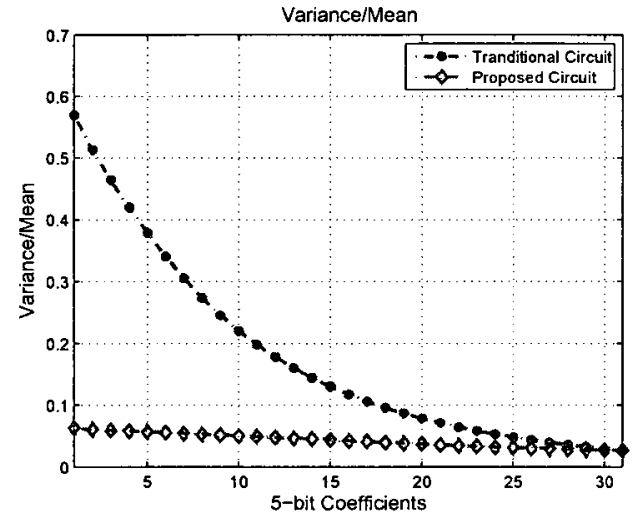

(b) Coeff $=15$

Figure 4.10: Error and Variance of the Output of a Current Source

difference in the average of the current source for the two circuits using the same coefficients, but the traditional circuit has a large variance. The reason for this is that the traditional circuit has a larger capacitance and thereby a larger charge share and feed through as compared with the proposed circuit. If the power supply of the circuit is an ideal voltage source, the current variance would not benefit to output (transmitted signals) of the circuit. In the real world, as the power supply has an impedance and both the trace on the PCB and the IC package have an impedance, the proposed circuit will have a better performance than the traditional circuit. For example, the bond wire of the IC package has an inductance of $1 \mathrm{nH} / \mathrm{mm}$. If the bond wire is $2 \mathrm{~mm}$, which is normal for a TQFP, the bond wire has a total inductance of $2 \mathrm{nH}$. The voltage drop of an inductor is given by:

$$
\Delta V=L \frac{d i}{d t}
$$

Figure 4.12 shows the voltage drop across the bond wire $(2 \mathrm{~mm})$ using the current shown in Figure 4.11. The proposed circuit has only a $0.07 \mathrm{~V}$ ripple compared to a $0.42 \mathrm{~V}$ voltage ripple in the traditional circuit for a coefficient of 9 without decoupling 


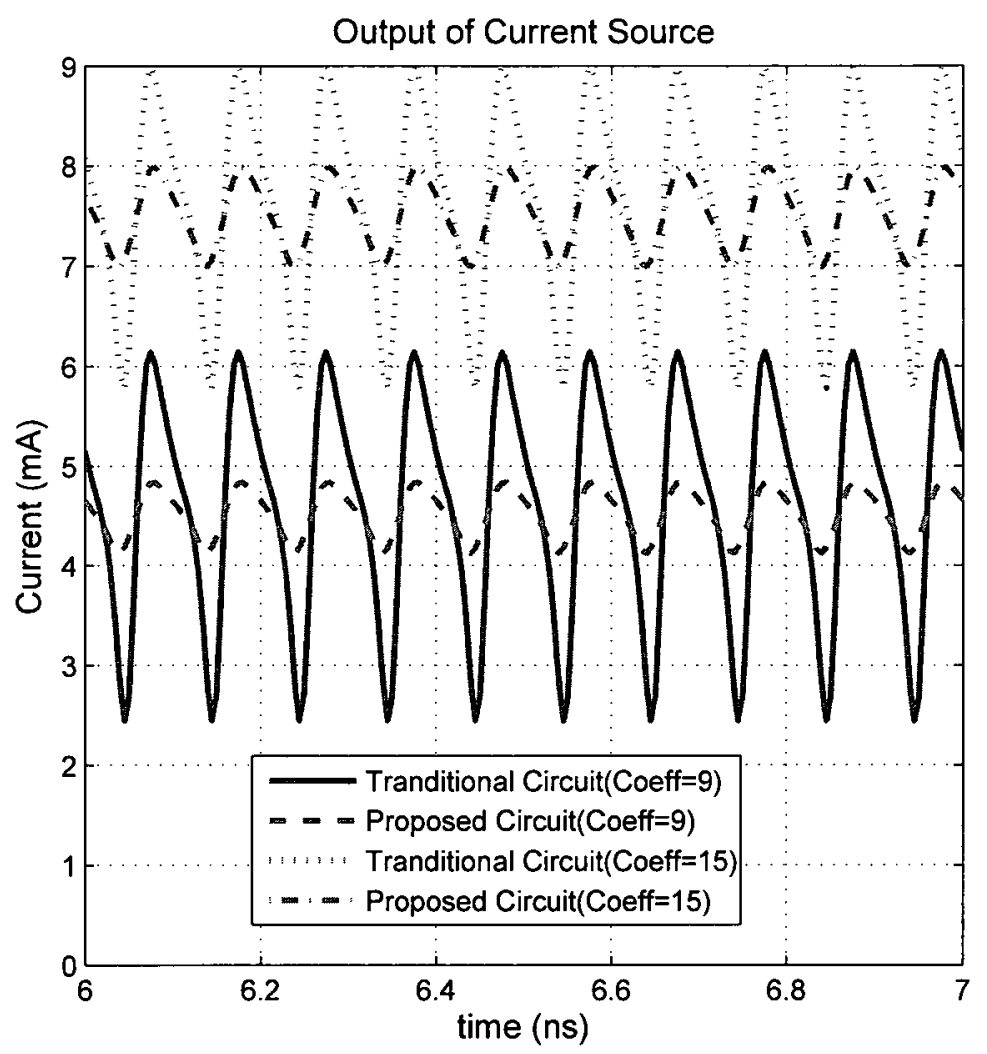

Figure 4.11: Output of a Current Source at Different Coefficients

capacitors. Of course, in the design, we have decoupling capacitors, and therefore the ripple will be reduced. The third benefit of using the proposed circuit is that all branches have the same current density, the voltage of the CMs are at the same voltage. Therefore the current of the CMs are the same because the output voltage of the CMs effects the current due to the limited output impedance of the CMs.

\subsection{2 iDAC Circuit}

In order to match the channel impedance, the load resistors are $50 \Omega$. For the DC coupling connection between a channel and a receiver, the total load impedance is 50 $\Omega / / 50 \Omega=25 \Omega$. For most industry standards, the CML output swing is between 


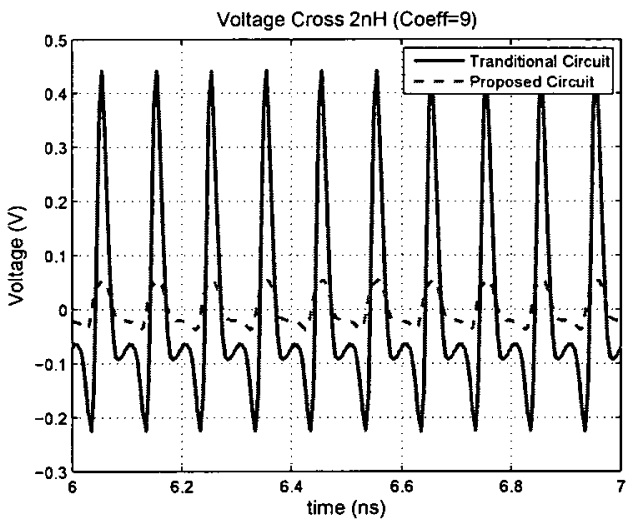

(a) Coeff $=9$

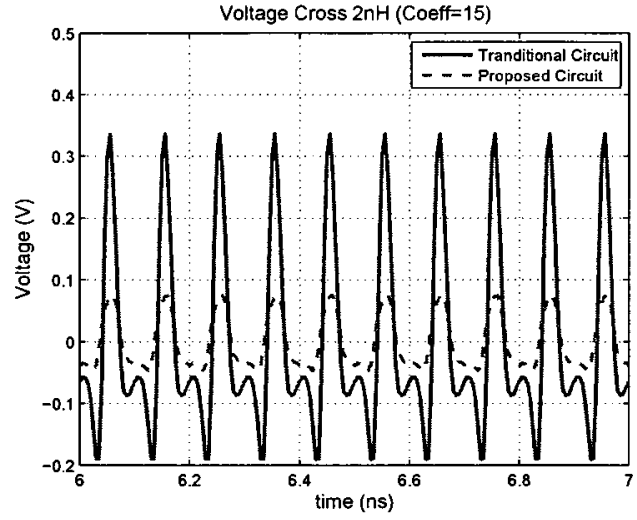

(b) Coeff $=15$

Figure 4.12: Voltage Drop through a $2 \mathrm{nH}$ Inductor at Different Coefficients

$400 \mathrm{mV}$ to $600 \mathrm{mV}$, which results in the differential output range from $800 \mathrm{mV}$ to $1.2 \mathrm{~V}$. In our design, we selected $1 \mathrm{~V}$ for the output range causing the differential output to change from $-0.5 \mathrm{~V}$ to $0.5 \mathrm{~V}$. For CML circuit, the output voltage is given by [Rogers2006]:

$$
V_{O}=I \times R_{\text {load }}
$$

To get $0.5 \mathrm{~V}$ at the output, the circuit requires a $20 \mathrm{~mA}$ current in total from the 5bit digitally controlled current source due to the impedance match of the channel. For $1 \mathrm{LSB}$, the current is $0.625 \mathrm{~mA}(20 \mathrm{~mA} / 32)$. If we use these parameters for the design, the maximum current of each tap is $20 \mathrm{~mA}$. From the optimized coefficients, we note that the maximum current of each tap cannot be $20 \mathrm{~mA}$ (in this case, the coefficients of all other taps are zero). The maximum possible values of the coefficients of each tap are not the same. In our application, we decided that the maximum current values for each tap are $16 \mathrm{~mA}, 8 \mathrm{~mA}, 8 \mathrm{~mA}, 4 \mathrm{~mA}, 2 \mathrm{~mA}, 2 \mathrm{~mA}$ from the main tap to tap5 respectively. 5-bit digitally controlled current sources are used but now 1 LSB is $0.5 \mathrm{~mA}(16 \mathrm{~mA} / 32)$. This improves the absolute resolution of the iDAC from 0.625 $\mathrm{mA}$ to $0.5 \mathrm{~mA}$ using the same digital circuit. 


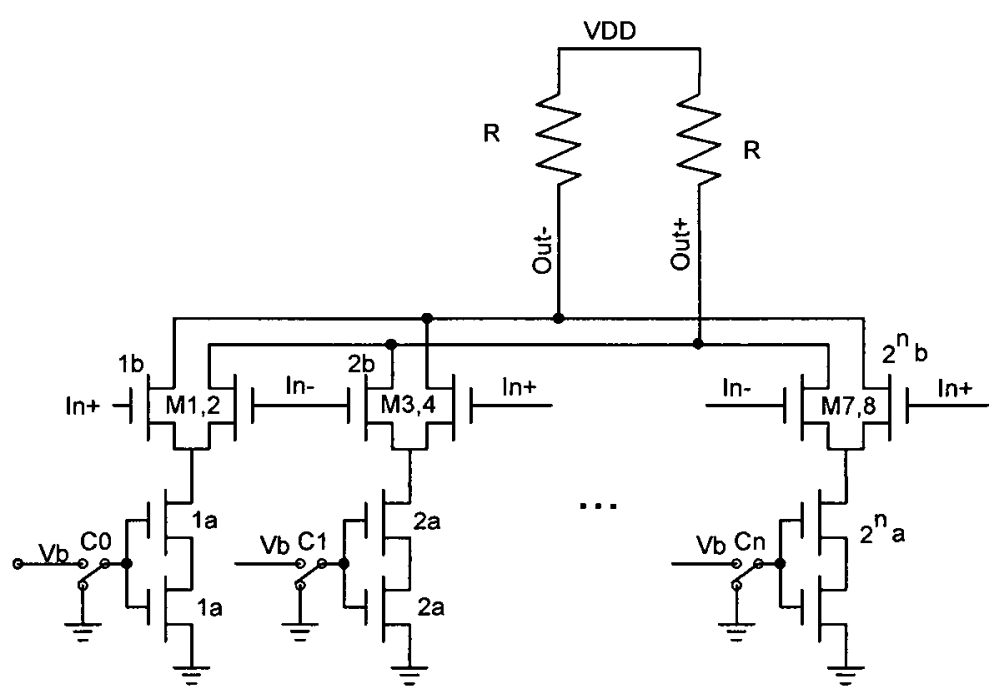

Figure 4.13: Pre-Emphasis Driver of One Tap

Figure 4.13 shows one tap of the pre-emphasis driver used in the proposed circuit. The number of branches depends on how many bits are required for that tap according to its maximum current. The benefit of using a different number of branches for each tap is the reduction of the total transistor size of the specific tap according to its requirement therefore reducing the current and power of the pre-driver circuit.

\subsection{Chip Interface and Memory Circuits}

The DFFs are used to store the values of the coefficients. The coefficients are constants for a given channel and data rate and they are stored while the chip is initialized. The clock of the DFFs for the input coefficients can be slow so the static CMOS circuit of the DFFs are selected in the interface circuit and storage elements. These DFFs are from the standard digital library of the chosen 90nm technology. Figure 4.14 shows the schematic of the interface and memory circuits. The coefficients are clocked in through several shift registers. The first data in is C50 and the last data in is Sig0. 


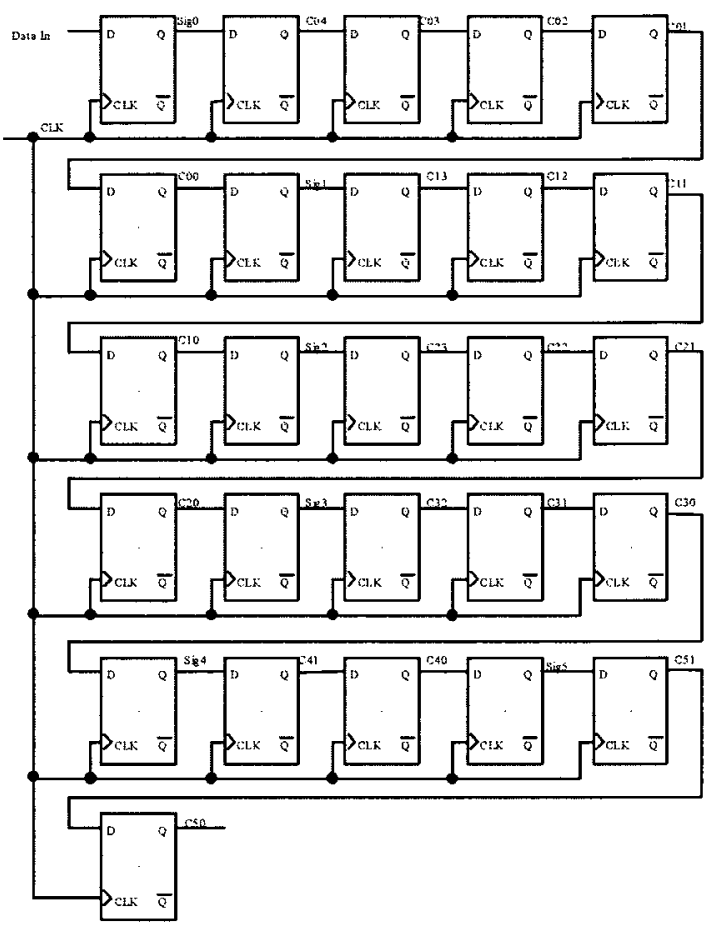

Figure 4.14: DFFs Used to Store the Value of the Coefficients

\subsection{Single to Differential Converter (STDC)}

To reduce the pin count of the chip, all data and clock signals are single ended. Inside the chip, the high speed data and clock circuits are CML and use differential signals. A circuit to convert the single ended signals to differential signals is required. Figure 4.15 shows a single-to-differential converter circuit.

\subsection{Layout}

Figure 4.16 shows the layout of the entire die.

Multi-finger transistors or multi small size transistors in parallel are used instead of one single large size transistor. This can reduce the mismatch. All transistors are of the same orientation to reduce the mismatch [33]. Another type of mismatch is 


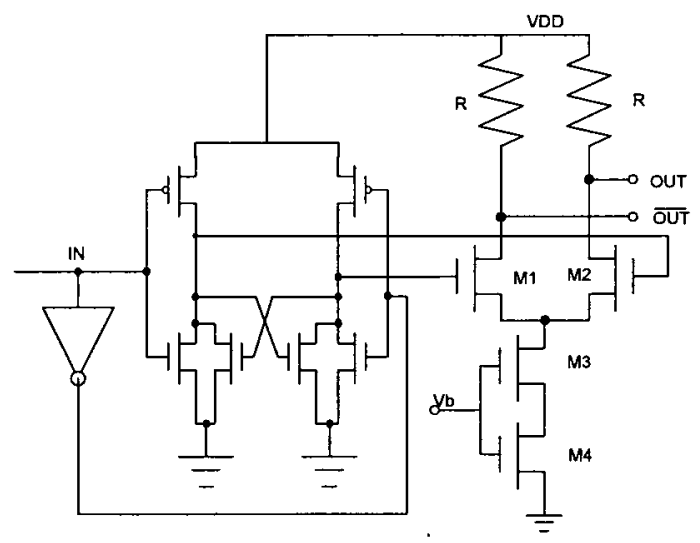

Figure 4.15: Single-to-Differential Signal Converter

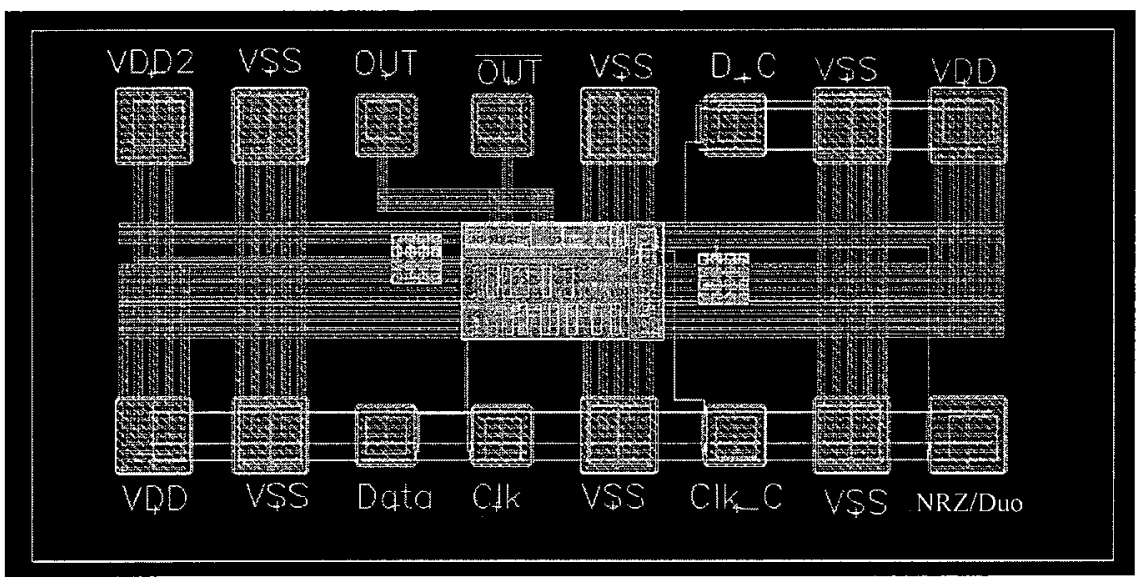

Figure 4.16: Layout View of the Die

caused by the presence or absence of other structures near the matched transistors. For example, the presence of the poly regions near the gate electrodes can cause slight variations in poly-silicon etch rates. These variations produce mismatches in the effective widths and lengths of the matched transistors. If similar dummy structures are placed near the channel, the mismatch can be reduced [33]. This is a reason why there are many of dummy transistors and resistors in this design.

Latch-up commonly exists in CMOS IC design and may lead to a circuit malfunction or could potentially damage the entire circuits. A latch-up circuit provides positive feed back and acts as as SCR to short the power supply to the ground. A 
large short circuit current between the power supply and ground can cause the circuit to malfunction or may damage the circuits due to heat [34]. To avoid latch-up, adding guard rings between the PMOS and NMOS is an effective way to reduce the parasitic resistance and prevent the formation of a positive feedback loop between the power supply and ground. Guard rings can also reduce interferance and noise between the different cells.

In order to reduce the interface and noise, two supplies are used. One power supply is for the FIR core circuits and the other is for the Pre-emphasis driver. Both power supplies use metal capacitors for decoupling capacitors. Some other nodes, such as the bias of the current source, use large size NMOS transistors as capacitors.

\subsection{Schematic and Post-Layout Simulation}

The test bench used for simulation is shown in Figure 4.17. The Device Under Test (DUT) block is the pre-emphasis circuit under test. The channel is defined by the .s4p file. There are two clock generator blocks. The block "10 GHz CLK" generates a $10 \mathrm{GHz}$ clock with a $50 \%$ duty cycle. This clock is connected to PRBS7 and the CLK pin of DUT. The " $500 \mathrm{MHz}$ CLK" block generates a $500 \mathrm{MHz}$ clock, which is used to clock in the coefficients as the chip is being initialized. The PRBS7 block generates PRBS7 data and is connected to the Data pin of the DUT. The "Coeff Generator" block generates the coefficients data signal and 26 clock cycles to initialize the chip according to the coefficients set by the simulator. The NRZ/Duo pin is connected to a switch which can select ground or VDD as its input. When the pin (NRZ/Duo) is connected to VDD, the DUT is set in the NRZ equalizer mode. When this pin is connected to ground, the DUT is set in the Duobinary equalizer mode. In the NRZ equalizer mode, the equalizer can be $\mathrm{BCE}$ or $\mathrm{BEE}$ depending on the coefficients initialized as the DUT is powered up. The channel at the transmitter side is connected 


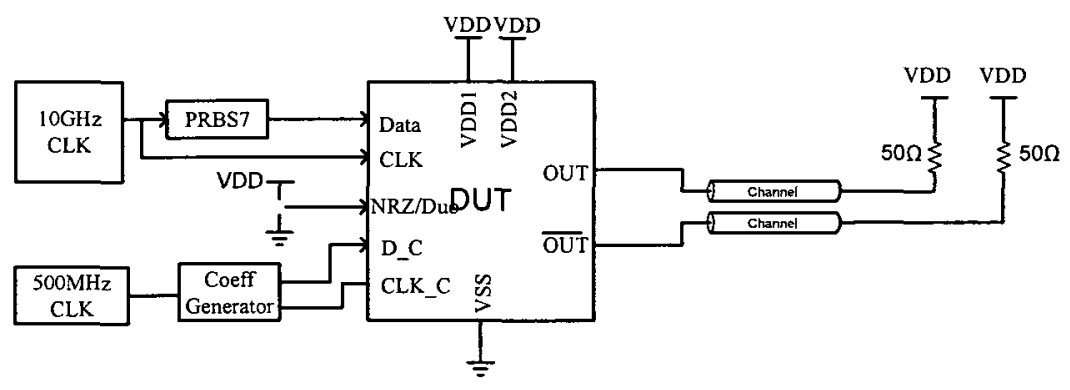

Figure 4.17: Test Bench for the Chip

to the output of the DUT and the receiver sided is connected to VDD through 50 Ohms terminating resistors. Figure 4.18 shows the simulation results for the schematic at different corners (PVT). The corners consider a $5 \%$ variation of the power supply and a temperature range from $0{ }^{\circ} \mathrm{C}$ to $85^{\circ} \mathrm{C}$.

From Figure 4.18, it is clear that the eye is closed at the transmitter side but at the receiver side, the eye is opened.

In order to compare the different corners more precisely, Table 4.3 quantitatively summaries the simulation results.

Table 4.3: Schematic Simulation Results

\begin{tabular}{|c|c|c|c|c|c|c|c|c|c|}
\hline \multicolumn{3}{|c|}{ Corner } & \multicolumn{2}{c|}{ Eye Opening } & \multicolumn{2}{c|}{ Current } & \multicolumn{3}{c|}{ Power } \\
\hline Process & VDD & Temperature & Vertical & Horizon & VDD1 & VDD2 & VDD1 & VDD2 \\
$(\mathbf{m V})$ & $(\mathrm{UI})$ & $(\mathbf{m A})$ & $(\mathbf{m A})$ & $\begin{array}{c}\text { Total } \\
(\mathbf{m W})\end{array}$ & $\begin{array}{c}(\mathrm{mW}) \\
(\mathbf{m W})\end{array}$ \\
\hline TT & 1.00 & 27 & 116.0 & 0.84 & 62.9 & 14.4 & 62.9 & 14.4 & 77.3 \\
\hline FF & 1.05 & 0 & 117.2 & 0.82 & 67.8 & 15.5 & 71.2 & 16.3 & 87.5 \\
\hline SS & 0.95 & 85 & 80.6 & 0.81 & 58.1 & 13.4 & 55.2 & 12.7 & 67.9 \\
\hline
\end{tabular}

From the Table 4.3 we can see that the corners have little effect on the horizontal eye opening but affects the vertical eye opening especially at the worst corner, which occurs with the slow process, high temperature and low voltage. Changing the voltage effects the vertical eye opening because the current reference changes as the voltage changes which is derived from power supply and a resistor. 


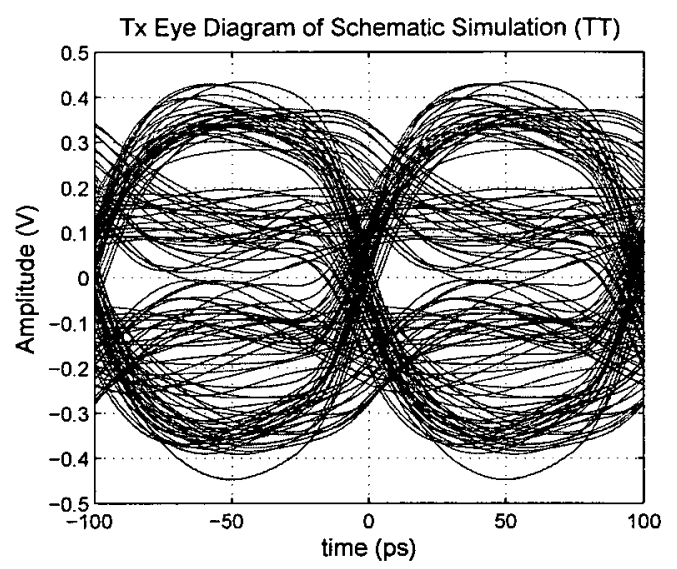

(a) $\mathrm{Tx}$

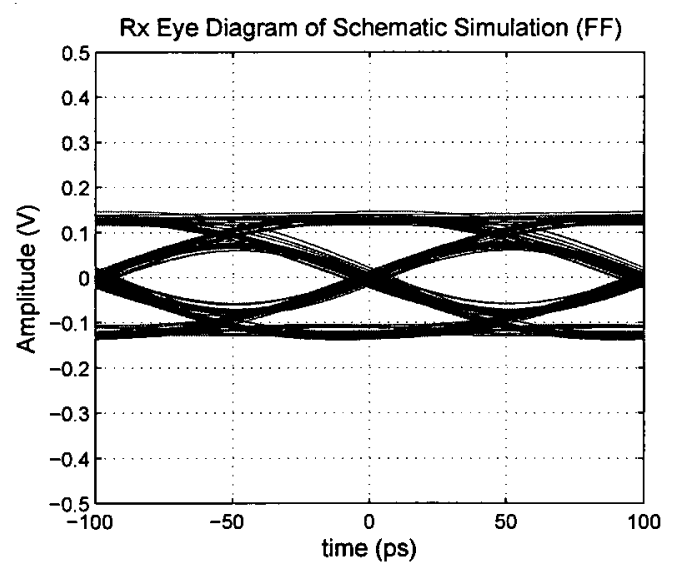

(c) $\mathrm{Rx} F \mathrm{~F}$

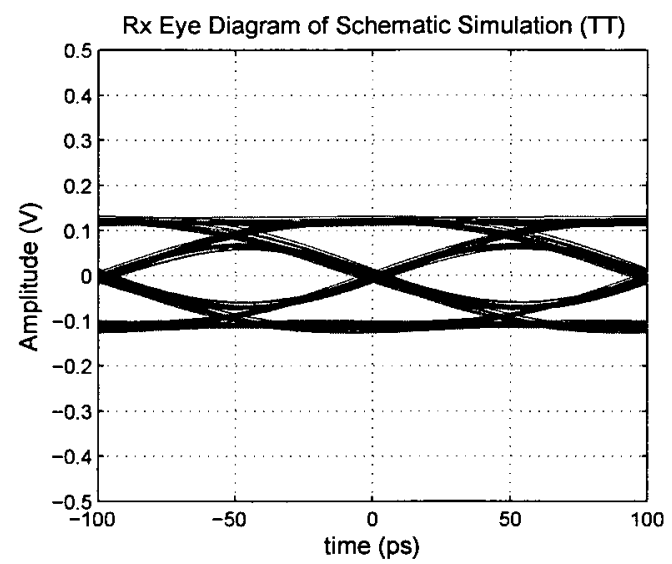

(b) $\mathrm{Rx} \mathrm{TT}$

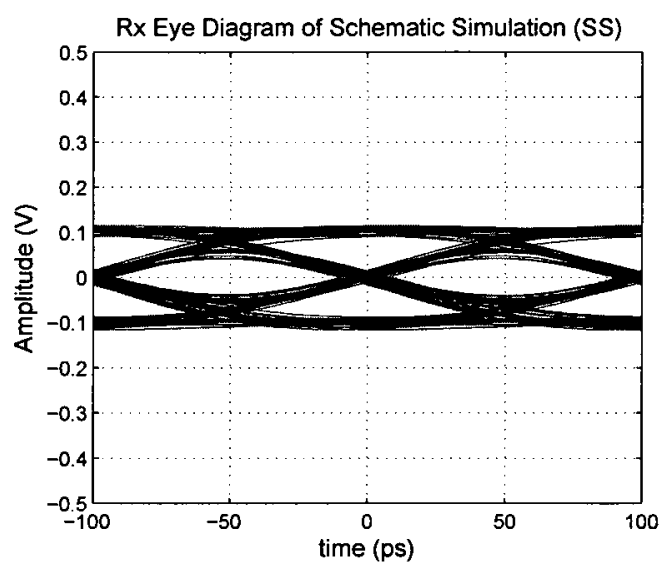

(d) Rx SS

Figure 4.18: Schematic Simulation

Figure 4.19 shows the post layout simulation eye diagram for different extraction strategies. The power supply is $1 \mathrm{~V}$.

A summary of post layout simulation results are listed in Table 4.4.

In comparing the results of schematic simulation and the post layout simulation, the eye opening of the post layout simulation is smaller than that of the schematic simulation. The reason for this is that there are significant parasitic resistance and capacitance on the connections between components. In the schematic simulation, the 


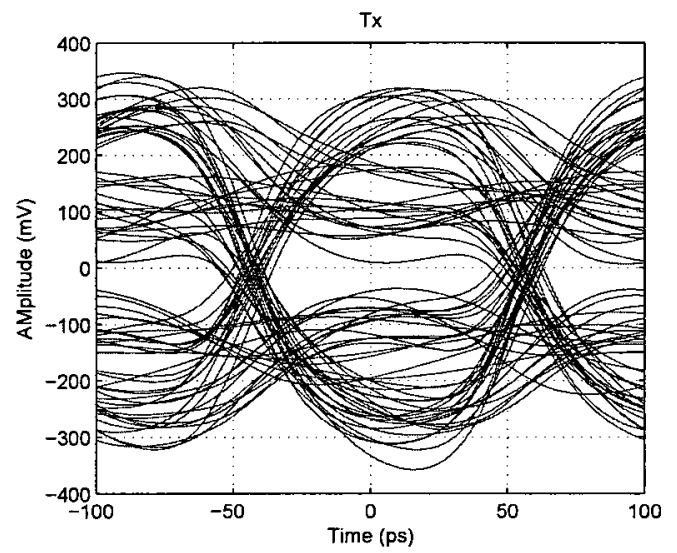

(a) $\mathrm{Tx}$

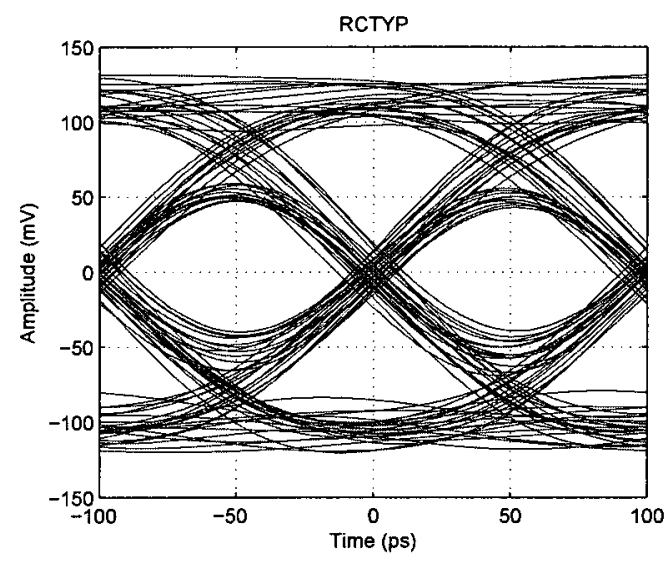

(c) RCTYP

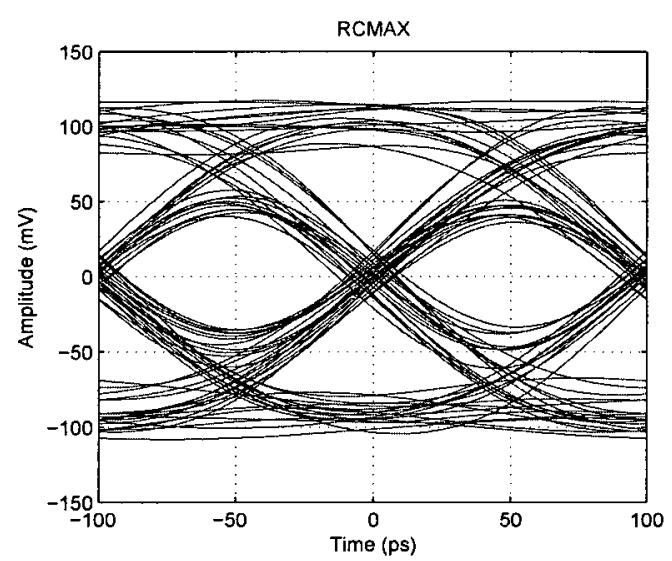

(b) RCMAX

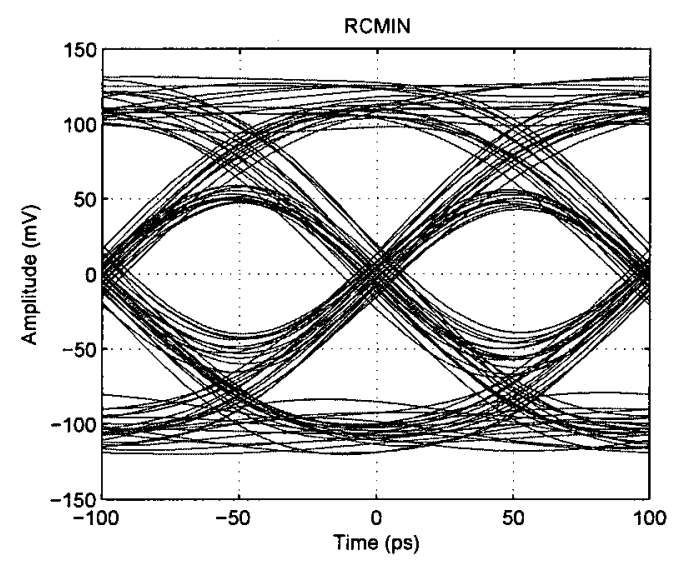

(d) RCMIN

Figure 4.19: Post Layout Simulation Using Different Extraction Strategies

connections between components is ideal meaning zero resistance and no parasitic capacitance. In the post layout simulation, the parasitic resistance and capacitance are extracted and these values are used to replace the ideal wires so the signal amplitude is reduced and it results in more jitter.

From the schematic simulation and post layout simulation, the results show that the proposed pre-emphasis circuit can compensate for the ISI up to the 10 Gbps data rate.

The total current for the 6-tap FIR pre-emphasis circuit is $76.3 \mathrm{~mA}$ and consumes 
Table 4.4: Post Layout Simulation Results

\begin{tabular}{|c|c|c|c|c|c|c|c|c|}
\hline \multicolumn{2}{|c|}{} & \multicolumn{2}{|c|}{ Eye Opening } & \multicolumn{2}{c|}{ Current } & \multicolumn{3}{c|}{ Power } \\
\hline Extract & VDD & Vertical & Horizon & VDD1 & VDD2 & VDD1 & VDD2 & Total \\
& $(\mathrm{V})$ & $(\mathrm{mV})$ & $(\mathrm{UI})$ & $(\mathrm{mA})$ & $(\mathrm{mA})$ & $(\mathrm{mW})$ & $(\mathrm{mW})$ & $(\mathrm{mW})$ \\
\hline RCMAX & 1.0 & 69.5 & 0.7560 & 62.9 & 14.4 & 62.9 & 14.4 & 77.3 \\
\hline RCTYP & 1.0 & 82.4 & 0.7583 & 61.9 & 14.4 & 61.9 & 14.4 & 76.3 \\
\hline RCMIN & 1.0 & 85.4 & 0.7602 & 61.1 & 14.4 & 61.1 & 14.4 & 75.6 \\
\hline
\end{tabular}

$76.3 \mathrm{~mW}$ under typical conditions. A comparison of the power dissipation of the proposed circuit with similar circuits of other papers is listed in Table 4.5. From Table 4.5, we can see that the power dissipation of the proposed circuit is lower as compared to some other designs. Although the lowest power dissipation is the design in [35] and [36], this work has 4 more taps than that of the design in [35] and 2 more taps than that of the design in [36]. Furthermore, the data rate in [35] is 6 Gbps, but the data rate in this work is $10 \mathrm{Gbps}$.

Table 4.5: Power Dissipation Comparison

\begin{tabular}{|c|c|c|c|c|c|}
\hline & Taps & Technology & Power $(\mathbf{m W})$ & VDD(V) & Data Rate(Gbps) \\
\hline$[\mathbf{3 7}]$ & 3 & $.18 \mu \mathrm{m}$ & 183.2 & 1.8 & $5 / 10$ \\
\hline$[\mathbf{3 5}]$ & 4 & $90 \mathrm{~nm}$ & 70 & $1.2 / 1.0$ & 10 \\
\hline$[38]$ & 4 & $.13 \mu \mathrm{m}$ & 180 & 1.5 & 10 \\
\hline$[39]$ & 4 & $90 \mathrm{~nm}$ & 95 & 1.2 & 10 \\
\hline$[36]$ & 2 & $.18 \mu \mathrm{m}$ & 40.5 & 1.8 & 6 \\
\hline This Work & 6 & $90 \mathrm{~nm}$ & 76.3 & 1.0 & 10 \\
\hline
\end{tabular}

\subsection{Chapter Summary}

The detailed design in $90 \mathrm{~nm}$ technology was discussed in this chapter. The design was verified by schematic and post layout simulation. From simulation, it is clear 
that the circuits can open the eye for channel B20 up to 10 Gbps. The typical total power dissipation of the entire pre-emphasis circuit is $76.3 \mathrm{~mW}$ and the maximum power dissipation of the circuit is $77.3 \mathrm{~mW}$. 


\section{Chapter 5}

\section{Test Setup and Measurement Results}

\subsection{Introduction}

The proposed pre-emphasis circuit was fabricated in ST Micro CMOS 90nm technology without package. The package provided by the Canadian Microelectronics Corporation (CMC) cannot work at a frequency of $10 \mathrm{GHz}$. This chapter describes the test bench setup and presents the measurement results.

\subsection{Test Bench Setup}

In the lab at Department of Electronics of Carleton University, there are two types of 8-pin probes used for testing. The part numbers are MCW-121-1 and MCW-121-2 respectively. The pin arrangement is shown in Figure 5.1.

Figure 5.2 shows the diagram of the test bench. The pre-emphasis circuit does not include the PRBS generator. We use an FPGA to generate PRBS7 at a low data rate of $50 \mathrm{Mb} / \mathrm{s}$ and $50 \mathrm{MHz}$ clock signal as the maximum frequency of the FPGA board used in the test is $50 \mathrm{MHz}$. The FPGA also generates the control bits and clock signal used to initialize the chip. The frequency of the clock for the initialization is 1 $\mathrm{MHz}$. 
MCW-121-2

\begin{tabular}{|c|c|c|c|c|c|c|c|}
\hline$\frac{\mathrm{O}}{\mathrm{O}}$ & $\stackrel{\oiiint}{\lessgtr}$ & $\frac{\sum_{\mathcal{J}}}{\omega}$ & $\frac{N}{N}$ & $\stackrel{\infty}{\infty}$ & 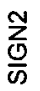 & $\stackrel{\mathscr{N}}{\lessgtr}$ & 只 \\
\hline \multicolumn{8}{|c|}{$M C W-121-1$} \\
\hline$\stackrel{\circ}{\stackrel{\circ}{S}}$ & $\stackrel{\mathscr{D}}{\supset}$ & $\frac{\bar{z}}{\infty}$ & $\frac{N}{\mathbb{N}}$ & $\ddot{2}$ & $\frac{N}{\frac{N}{N}}$ & $\mathscr{0}$ & $\stackrel{\circ}{9}$ \\
\hline
\end{tabular}

Figure 5.1: Pin Arrangement of 8-Pin Probes

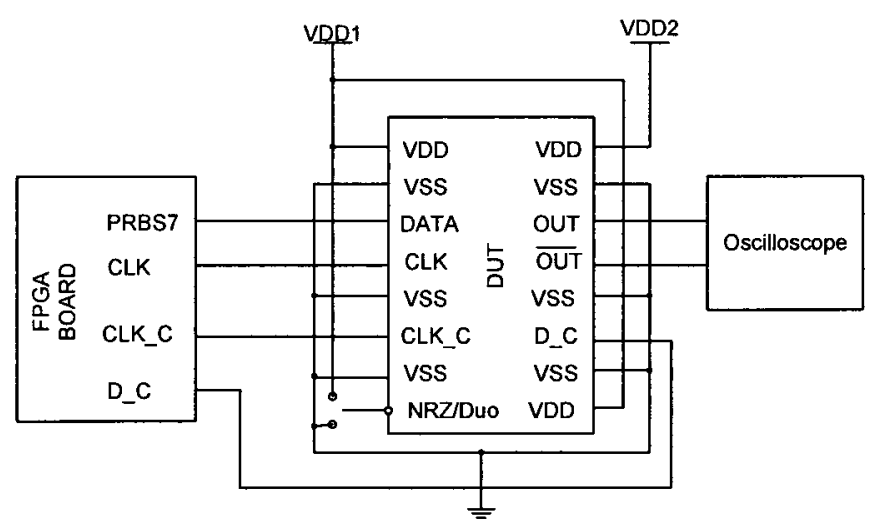

Figure 5.2: Test Bench Using Lab Equipment

Figure 5.3 shows the chip die (a) with the probes and (b) the connection of the test instrument. The die has 8 pins on each side of the chip.

\subsection{Measurement Results}

As a channel does not exist in the lab and we only implemented the transmitter, a receiver circuit is not available to test the eye at the receiver side. Therefore we only test the circuits at lower frequencies to verify the function of the circuits.

The goal of the test is to verify that the current of each tap can be controlled through changing the value of the coefficients. Figure 5.4 shows the output of the 


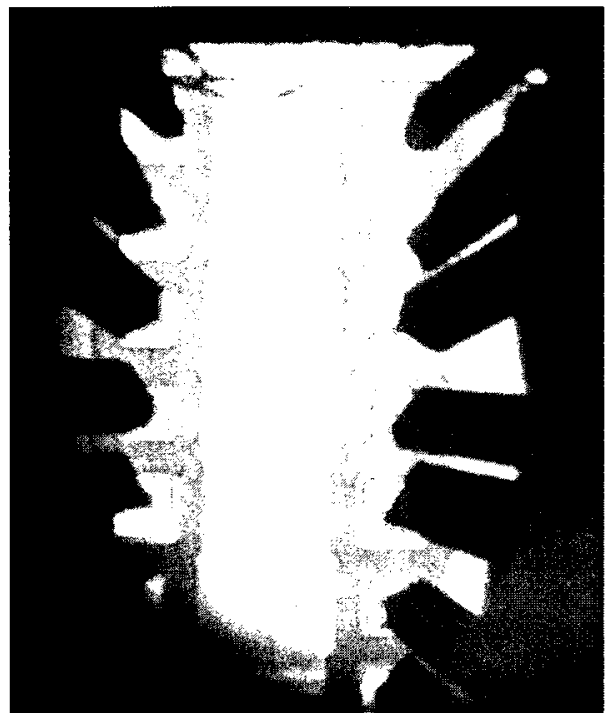

(a) Die With Two 8-Pin Probes

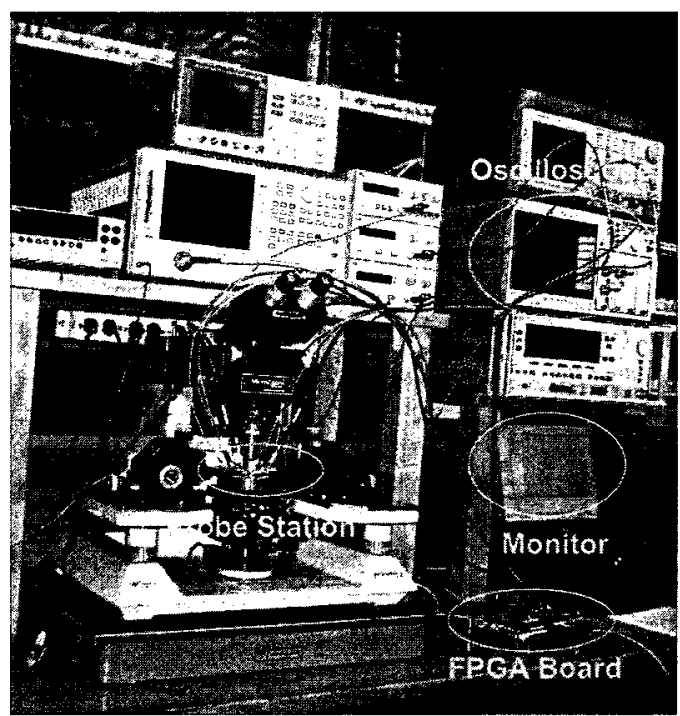

(b) Test Instruments and DUT

Figure 5.3: Chip Test In the Lab

pre-emphasis circuit when the Coeff0 is set to 16 and all others are set to 0 (Figure $5.4(\mathrm{a}))$ and the Coeff 1 is set to 8 and all others are set to 0 (Figure $5.4(\mathrm{~b})$ ). In Figure 5.4 , point $\mathrm{A}$ is the $\mathrm{DC}$ zero volts of the output signals $O U T$ and $\overline{O U T}$, and point $\mathrm{B}$ is the zero volts of the differential output signal $O U T-\overline{O U T}$.

Table 5.1 summarizes the test results for each iDAC bit in the output amplitude. Table 5.1 shows that the maximum relative error is $-9.62 \%$ when Bit2 of Tap3 is set to 1 . Most results indicated that the relative error is less $3 \%$, but there are 9 bits for which the relative error is more than $3 \%$. A possible reason for this is the possible mismatch of the transistors and the noise. In the future, deep N-well should be added to isolate the noise coupling. The Well Proximity Effect (WPE) should also be considered in the layout design.

The measured current for VDD1 (FIR Core) is $40.6 \mathrm{~mA}$ at a data rate of $50 \mathrm{Mbps}$ and the current for VDD2 (pre-emphasis driver output) is $14.3 \mathrm{~mA}$. The power supply voltage of both VDD1 and VDD2 are $1 \mathrm{~V}$. The total power dissipation is $54.9 \mathrm{~mW}$ 
at a data rate of $50 \mathrm{Mbps}$.

Table 5.1: Measured Differential Output Signal Value vs Coefficients Value

\begin{tabular}{|c|c|c|c|c|}
\hline Coeff & Bit & $\begin{array}{c}\text { Measured Output } \\
(\mathbf{m V})\end{array}$ & $\begin{array}{c}\text { Desired (mV) } \\
(\mathrm{mV})\end{array}$ & $\begin{array}{c}\text { Relative Error } \\
(\%)\end{array}$ \\
\hline \multirow{5}{*}{ o } & 4 & 788.12 & 800.0 & -1.49 \\
\hline & 3 & 404.68 & 400.0 & 1.17 \\
\hline & 2 & 199.26 & 200.0 & -0.37 \\
\hline & 1 & 101.54 & 100.0 & 1.54 \\
\hline & 0 & 50.692 & 50.0 & 1.38 \\
\hline \multirow{4}{*}{1} & 3 & 400.68 & 400.0 & 0.17 \\
\hline & 2 & 190.74 & 200.0 & -4.63 \\
\hline & 1 & 93.148 & 100.0 & -6.85 \\
\hline & 0 & 50.898 & 50.0 & 1.80 \\
\hline \multirow{4}{*}{2} & 3 & 379.94 & 400.0 & -5.02 \\
\hline & 2 & 183.37 & 200.0 & -8.32 \\
\hline & 1 & 98.380 & 100.0 & -1.62 \\
\hline & 0 & 48.896 & 50.0 & -2.21 \\
\hline \multirow{3}{*}{3} & 2 & 180.76 & 200.0 & -9.62 \\
\hline & 1 & 106.50 & 100.0 & 6.50 \\
\hline & 0 & 50.194 & 50.0 & 0.39 \\
\hline \multirow{2}{*}{4} & 1 & 94.306 & 100.0 & -5.69 \\
\hline & 0 & 51.770 & 50.0 & 3.54 \\
\hline \multirow{2}{*}{5} & 1 & 93.598 & 100.0 & -6.40 \\
\hline & 0 & 49.922 & 50.0 & -0.16 \\
\hline
\end{tabular}




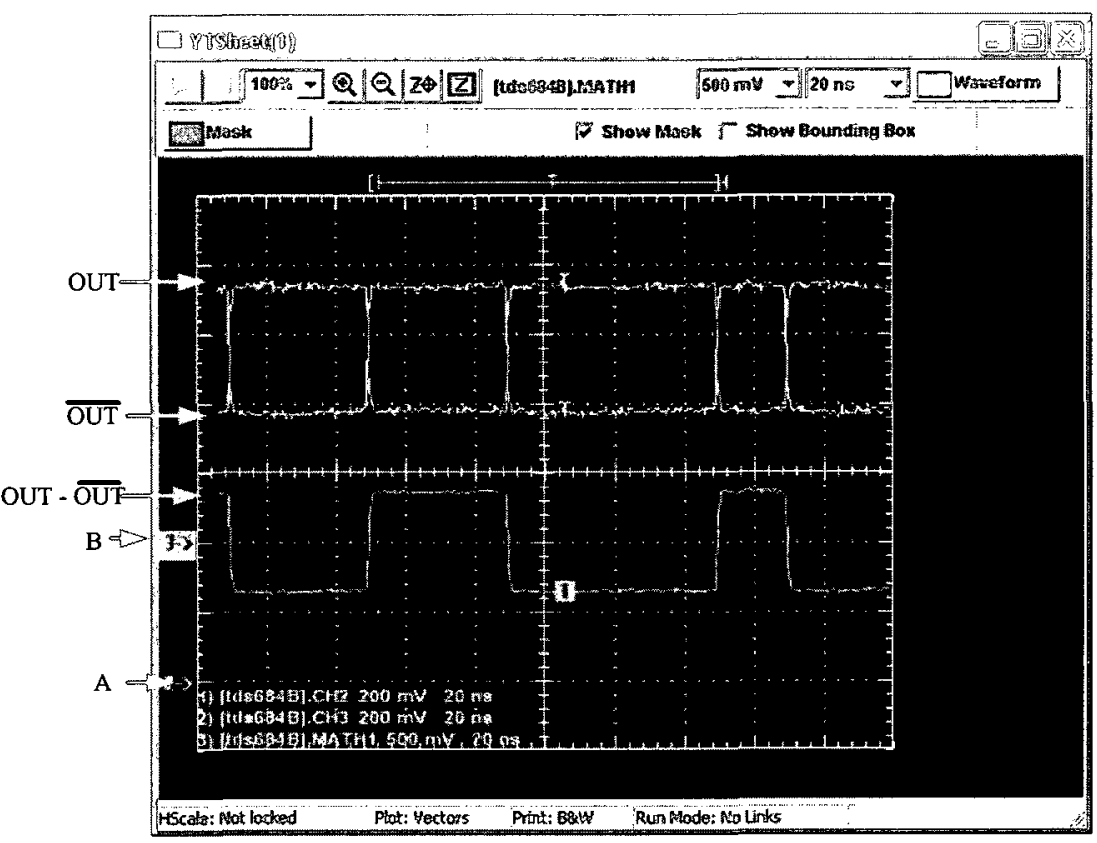

(a) Bit4 $=1$ (Coeff0)

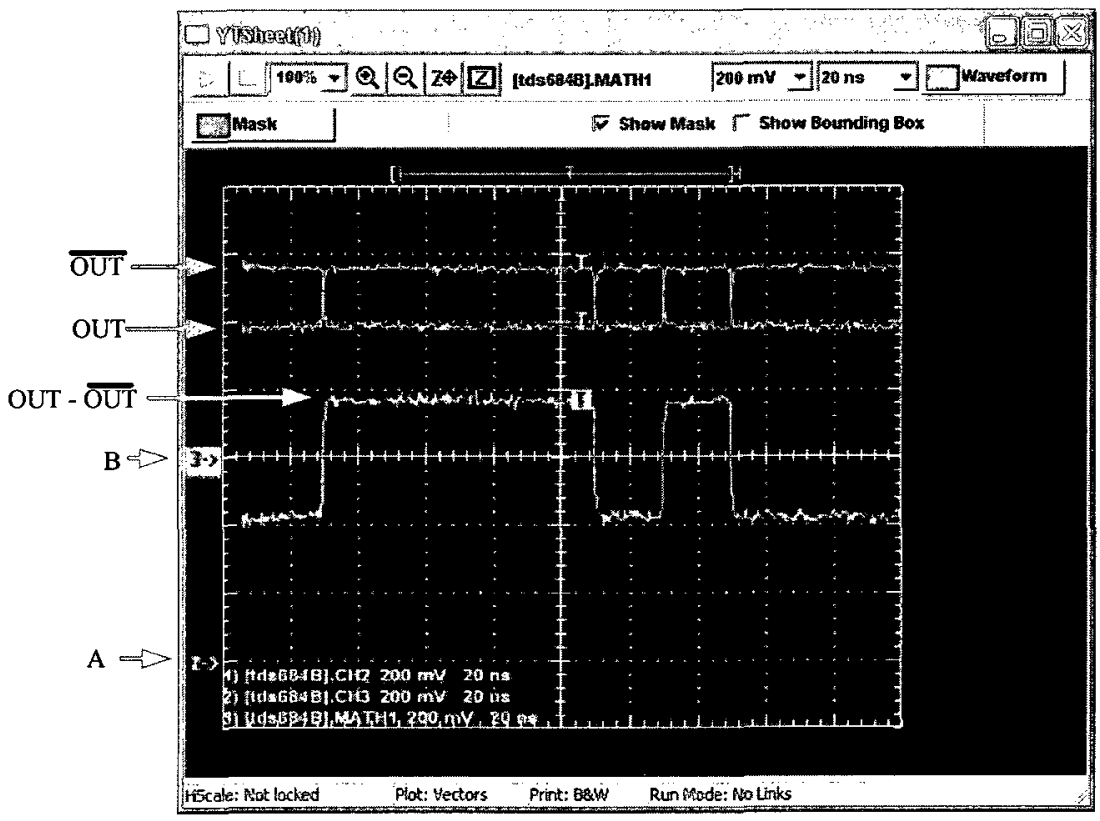

(b) Bit3 = 1 (Coeff1)

Figure 5.4: Test Result of the Output Signals 


\section{Chapter 6}

\section{Conclusion, Contribution and Future}

\section{Work}

\subsection{Conclusions}

Equalizer circuits are widely used in high data rate digital communication systems, such as board-to-board communication and chip-to-chip communication. With the demand for high data rates and low system cost over a high lossy channel, the challenge of designing a more efficient equalizer, including the algorithm and hardware, increases.

In this Thesis, the theory of the equalizer and the method of optimizing the coefficients are discussed in the Chapter 3. Using this method, a digital equalizer for the transmitter side was designed in 90nm CMOS technology and the chip was fabricated. A new design method used to reduce the capacitance of the line driver stage is presented. The simulation results show that the $C_{g d}$ and $C_{g s}$ can be reduced when the transistors turn on. The reduced value depends on the coefficients used for the equalizer. When the coefficients are $23,-13,2,0,-1,0$ from the main tap to the sixth tap respectively, the $C_{g d}$ changes from $619 \mathrm{fF}$ (traditional circuit) to 506 $\mathrm{fF}$ (proposed circuit), a reduction of $18.3 \% ; C_{g s}$ changes from $207 \mathrm{fF}$ (traditional 
circuit) to $195 \mathrm{fF}$ (proposed circuit), a reduction of $5.8 \%$. The test results show that the coefficients of the 6-tap circuit are programmable through a serial communication cable. The power of the entire chip is $54.9 \mathrm{~mW}$ (measured at a data rate of $50 \mathrm{Mbps}$ ). The eye at the receiver side is open (post layout simulation, worst case, vertical 69.5 $\mathrm{mV}$ and horizontal $0.756 \mathrm{UI})$.

\subsection{Contribution}

The contributions of this Thesis are listed as the following:

- A new design method for reducing the capacitance of the driver stage of the pre-emphasis circuit is developed and verified by simulation. Using this method either the performance can be improved while maintaining the same power dissipation or the power dissipation is reduced with the same performance.

- A complete FIR circuit for pre-emphasis was designed in 90nm CMOS technology. The FIR filter with a programmable 6-tap circuit can be easily configured for different channels and different algorithms to optimize the eye diagram at the receiver.

- The circuit was fabricated with ST Micro 90nm CMOS technology. The test results verify the function of the circuit.

\subsection{Future Work}

In the future, the design of the transceiver including the receiver and clock management unit (CMU) are the next step for the Professor Kwasniewski's group. The PRBS7 should also be included in the chip for the test purposes. An advanced layout is another important consideration for future work. 


\subsection{Publications}

During the period of my study and thesis research at Carleton University, 7 published papers as listed below, summarize my research and my major contributions in the area of study.

1. Dezhong Cheng, Bangli Liang, Dianyong Chen, Tad Kwasniewski, "Optimize Transistor Size for FIR Pre-emphasis with Programmable Coefficients," 14th Asia-Pacific Conference on Communications (APCC08), pp.1-5, October, 2008.

2. Dezhong Cheng, Bangli Liang, Dianyong Chen, Tad Kwasniewski, "A Reduced Power 6-Tap Pre-Emphasis for 10Gb/s Backplane Communications," 24th Queen's Biennial Symposium on Communications, pp.93-96, June, 2008.

3. Bangli Liang, Dianyong Chen, Bo Wang, Dezhong Cheng, Tad Kwasniewski, "A 43-GHz Static Frequency Divider in 0.13 $\mu \mathrm{m}$ Standard CMOS," IEEE Canadian Conference on Electrical and Computer Engineering 2008 (CCECE'08)Circuits, Devices and Systems, pp.111-114, May, 2008.

4. Bangli Liang, Tad Kwasniewski, Dianyong Chen, Dezhong Cheng "A 42-Gb/s Decision Circuit in $0.13 \mu \mathrm{m}$ CMOS," Sixth Annual Conference on Communication Networks and Services Research(CNSR'08), pp.339-343, May, 2008.

5. Bangli Liang, Tad Kwasniewski, Dianyong Chen, Bo Wang, Dezhong Cheng, "1V Supply CMOS DEMUX for 40-Gb/s Optical Communication Systems," 24th Queen's Biennial Symposium on Communications, pp.154-157, June, 2008.

6. Bangli Liang, Tad Kwasniewski, Zhigong Wang, Dianyong Chen, Bo Wang, Dezhong Cheng, "0.13 $\mu \mathrm{m}$ 1.0V-1.5V Supply Digital Blocks for 40-Gb/s Optical Communication Systems," 2008 International Conference on Communications: Circuits and Systems (ICCCAS'08), pp.1393-1397, May, 2008. 
7. Bangli Liang, Tad Kwasniewski, Zhigong Wang, Dianyong Chen, Bo Wang, Dezhong Cheng, "A Monolithic 10-Gb/s CMOS Limiting Amplifier for Low Cost Optical Communication Systems," 14th Asia-Pacific Conference on Communications (APCC08), pp.1-4, May, 2008.

8. Dianyong Chen, Bo Wang, Bangli Liang, Dezhong Cheng, and Tad Kwasniewski, "A Novel CMOS Edge Equalizer for 10-Gb/s Highly Lossy Backplane," 24th Queen's Biennial Symposium on Communications, pp.294-297, June, 2008.

9. Dianyong Chen, Bo Wang, Bangli Liang, Dezhong Cheng and Tad Kwasniewski, "Decision-Feedback-Equalizer for 10-Gb/s Backplane Transceivers for Highly Lossy 56-inch Channels," 2008 International Conference on Communications: Circuits and Systems (ICCCAS'08), pp.668-672, May, 2008. 


\section{List of References}

[1] B. Brunn and S. Anderson, "Edge-Equalization Extends Performance in Multi-gigabit Serial Links." Design Conference 2005, available at http://www.designcon.com/infovault/paper.asp.

[2] "Common Electrical I/O CEI - Electrical and Jitter Interoperability Agreements for $6 \mathrm{G}+$ bps and $11 \mathrm{G}+$ bps IO." available at http://www. oiforum. com/public/impagreements.html.

[3] "IEEE P802.3ap Backplane Ethernet Task Force." available at http://www . ieee802.org/3/ap/.

[4] "IEEE P802.3ba $40 \mathrm{~Gb} / \mathrm{s}$ and $100 \mathrm{~Gb} / \mathrm{s}$ Ethernet Task Force." available at http://www . ieee802.org/3/ba/.

[5] D. Chen, B. Wang, B. Liang, D. Cheng, and T. Kwasniewski, "A Novel CMOS Edge Equalizer for 10Gb/s High Lossy Backplane," in Biennial Symposium on Communication, pp. 294-297, 2008.

[6] W. Peter, "IEEE P802.3ap Task Force Channel Model Material." available at http:grouper. ieee.org/groups/802/3/ap/public/channel model/index.html.

[7] D. Chen, B. Wang, B. Liang, D. Cheng, and T. Kwasniewski, "An Improved Simulation Method for High-Speed Data Transmission through Electrical Backplane," in Microsystems and Nanoelectronics Research Conference, pp. 101-104, 2008.

[8] K. C. Cupta, R. Gard, and I. Bahl, Microstrip Lines and Slotlines. Dedham, MA: Artech House, Inc., 1979.

[9] H. Wheeler, "Transmission Line Properities of Parallel Strips Seperated by Dielectric Sheet," IEEE Trans. Microwave Theory Tech, vol. MTT-13, pp. 172-185, 1965. 
[10] D. Cheng, B. Liang, D. Chen, and T. Kwasniewski, "A Reduced Power 6-Tap Pre-Emphasis for 10Gb/s Backplane Communications," in Biennial Symposium on Communication, pp. 93-96, 2008.

[11] Y. Huang, Q. Chen, and T. Lee, "A 4-PAM Adaptive Analog Equalizer for Backplane Interconnections," in VLSI Design, Automation and Test, pp. 200203, 2008.

[12] J. Lee, M. Chen, and H. Wang, "Design and Comparison of Three 20-Gb/s Backplane Transceivers for Duobinary, PAM4, and NRZ data," IEEE Journal of Solid-State Circuits, vol. JSSC-43, pp. 2120-2133, 2007.

[13] S. Gondi, J. Lee, D. Takeuchi, and B. Razavi, "A 10Gb/s CMOS Adaptive Equalizer for Backplane Applications," in Internal Solid-State Circuits Conference, pp. 328-330, 2005.

[14] J. Chen, G. Sheets, C. Guo, F. Saibi, F. Yang, K. Azadet, J. Lin, and G. Zhang, "Electrical Backplane Equalization Using Programmable Analog Zeros and Folded Active Inductors," in Circuits and Systems, pp. 1366-1369, 2005.

[15] E. Ori, R. Adee, M. Amir, H. Yaniv, and L. D. M. Miki, "An Adaptive 4Tap Analog FIR Equalizer for 10Gb/s over Backplane Serial Link Receiver," in European Solid-State Circuits Conference, pp. 178-181, 2008.

[16] R.Payne, P. Landman, B. Bhakta, S. Ramaswamy, S. Wu, J. Power, M. Erdogan, A. Yee, R. Gu, L. Wu, Y. Xie, B. Parthasarathy, K. Brouce, W. Mohammed, K.Heragu, V. Gupta, L. Dyson, and W. Lee, "A 6.25Gb/s Binary Transceiver in $0.13 \mu \mathrm{m}$ CMOS for Serial Data Transmission Across High Loss Legacy Backplane Channel," IEEE Journal of Solid-State Circuits, vol. 40, pp. 2646-2657, 2005.

[17] H. Kim, A. Raghavan, E. Gebara, and J. Laskar, "Backplane Equalization Comparison for $10 \mathrm{~Gb} / \mathrm{s}$ Data Communication with $0.25 \mu \mathrm{m}$ SiGe BiCMOS and $0.18 \mu \mathrm{m}$ CMOS Feed-Forward Equalizers," in Wireless Technologies, pp. 259-362, 2007.

[18] J. Chen and T. Kwasniewski, "A 6.25Gb/s Pipelined Half-Rate Decision Feedback Equalizer for High Speed Backplane Data Communication," in NEWCAS Conference, pp. 127-130, 2005.

[19] M. Li, W. Huang, S. Wang, and T. Kwasniewski, "0.18 $\mu \mathrm{m}$ CMOS Backplane Receiver with Decision-Feedback Equalization Embedded," Electronics Letters, vol. 42, pp. 752-754, 2006. 
[20] M. Zhou, E. Zhu, S. Wang, and Z. Wang, "A 6.26Gb/s Decision Feedback Equalizer Used in SerDes for High-Speed Backplane Communication," in Microwave and Millimeter Wave Technology, pp. 1-4, 2007.

[21] S. Chandramouli, F. Bien, K. Hyoungsoo, E. Gebara, and J. Laskar, "A 10Gb/s Unclocked Current-Mode Logic (CML) Analog Decision-Feedback Equalizer (ADFE) in $0.18 \mu \mathrm{m}$ CMOS," in European Solid State Circuits Conference, pp. $512-515,2007$.

[22] A. Amirkhany, A. Abbasfar, V. Stojanovic, and M. Horowaitz, "Analog MultiTone Signaling for High-Speed Backplane Electrical Links," in Global Telecommunication Conference, pp. 1-6, 2006.

[23] N. Krishnapura, M. Barazande-Pour, Q. Chaudhry, J. Khoury, K. Lakshmikumar, and A.Aggarwal, "A 5Gb/s NRZ Transceiver with Adaptive Equalization for Backplane Transmission," in International Solid-State Circuits Conference, pp. 60-63, 2005.

[24] P. Landman, K. Brouse, V. Gupta, W. Song, R. Payne, U. Erdogan, R. Gu, A.L. Yee, B. Parthasarathy, S.Ramaswamy, B.Bhakta, W.Mohammed, J. Powers, Y. Xie, L. Wu, L.Dyson, K.Heragu, and W. Lee; "A Transmit Architecture with 4-Tap Feedforward Equalization for 6.25/12.5Gb/s Serial Backplane Communications," in Internal Solid State Circuits Conference, pp. 68-70, 2005.

[25] J. Bulzacchelli, M. Meghelli, S. Rylov, W. Rhee, A. Rylyakov, H. Ainspan, B. Parker, M. Beakes, A. Chung, T. Beukema, P. Pepeljugoski, L. Shan, Y. Kwark, S. Gowda, and D. Friedman, "A 10-Gb/s 5-Tap DFE/4-Tap FFE Transceiver in 90-nm CMOS Technology," IEEE Journal of Solid-State Circuits, vol. 41 , pp. $2885-2900,2006$.

[26] L. Zhang and T. Kwasniewski, "Using Bit-Edge Equalization in Highspeed Backplane Data Transmission," in International Conference on Communications and Networking, pp. 642-646, 2008.

[27] M. Li, S. Wang, Y. Tao, and T. Kwasniewski, "FIR Filter Optimization as Pre-Emphasis of High-Speed Backplane Data Transmission," IEEE Electronics Letters, vol. 40, pp. 773-776, 2004.

[28] J. W. Bergmans, Digital (Baseband) Transmission and Recording. Boston: Kluwer Academic Publishers. 
[29] R.Payne, B. Bhakta, S. Wu, J. Power, P. Landman, M. Erdogan, A. Yee, L. Wu, Y. Xie, B. Parthasarathy, K. Brouce, W. Mohammed, K.Heragu, V. Gupta, L. Dyson, and W. Lee, "A 6.25Gb/s Binary Adaptive DFE with First Postcursor Tap Cancellation for Serial Backplane Communications," in International Solid-State Circuits Conference, pp. 68-70, 2005.

[30] J. Rogers, C. Plett, and F. Dai, Integrated Circuit Design for High-Speed Frequency Synthesis. Norwood, MA: Artech House, Inc., 2006.

[31] P. Gray, P. Hurst, S. Lewis, and R. Meyer, Analysis and Design of Analog Integrated Circuits. Norwood, MA: John Wilry and Sons, Inc., 2000.

[32] T. Dickson, K. Yau, T. Chalvatzis, A. Mangan, E. Laskin, R. Beerkens, P. Westergaard, M. Tazlauanu, M.-T. Yang, and S. Voinigescu, "The Invariance of Characteristic Current Densities in Nanoscale MOSFETs and Its Impact on Algorithmic Design Methodologies and Design Porting of $\mathrm{Si}(\mathrm{Ge})$ (Bi)CMOS High-Speed Building Blocks," IEEE Journal of Solid-State Circuits, vol. 44, pp. 1830-1845, 2006.

[33] A. Hasting, The Art of Analog Layout. Upper Saddle River, NJ: Prentice Hall., 2001.

[34] D. A. John and K. Martin, Analog Integrated Circuit Design. John Willey and Sons Inc., 1997.

[35] J. Bulzacchelli, M. Meghelli, S. V. Rylov, W. Rhee, A. Rylyako, H. Ainspan, B. Parker, M. Beakes, A. Chung, T. Beukema, P. Pepeljugoski, L. Shan, Y. Kwark, S. Gowda, and D. Friedman, "A 10Gb/s 5-Tap DFE/4-Tap FFE Transceiver in 90nm CMOS Technology," IEEE Journal of Solid-State Circuits, vol. 41, pp. 2646-2657, 2006.

[36] C. Chu, C. Chuang, C.H.Lin, and S. Jou, "A 6Gb/s Serial Link Transmitter with Pre-Emphasis," in International Symposium on VLSI Design, Automation and Test, pp. 1-2, 2007.

[37] C. Lin and S. Jou, "4/2 PAM Pre-Emphasis Teansmitter with Combined Driver and Mux," in Asian Conference on Solid-State Circuits Conference, pp. 189-192, 2005.

[38] F. Weiss, D. Kehrer, and A. Scholtz, "Transmission over Lossy Copper Channels for $10 \mathrm{~Gb} / \mathrm{s}$ in $0.13 \mu \mathrm{m}$ CMOS," in Radio Frequency Integrated Circuits Symposium, pp. 11-13, 2006. 
[39] A. Rylyakov and S. Rylov, "A Low Power 10Gb/s Serial Link Transmitter in 90nm CMOS," in Compound Semiconductor Integrated Circuit Symposium, pp. 189-192, 2005. 


\section{Appendix A}

\section{Schematic of Pre-emphasis Circuits}

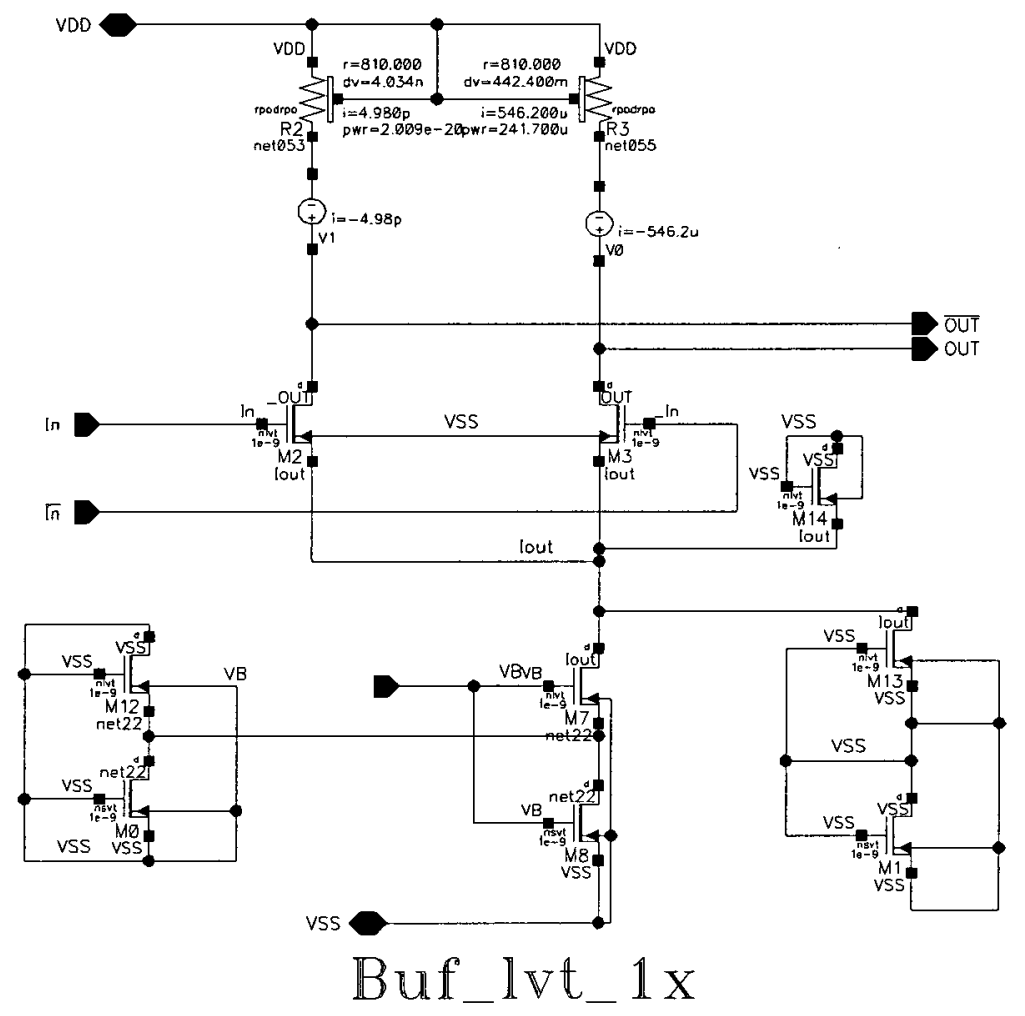

Figure A.1: Schematic of Buffer 


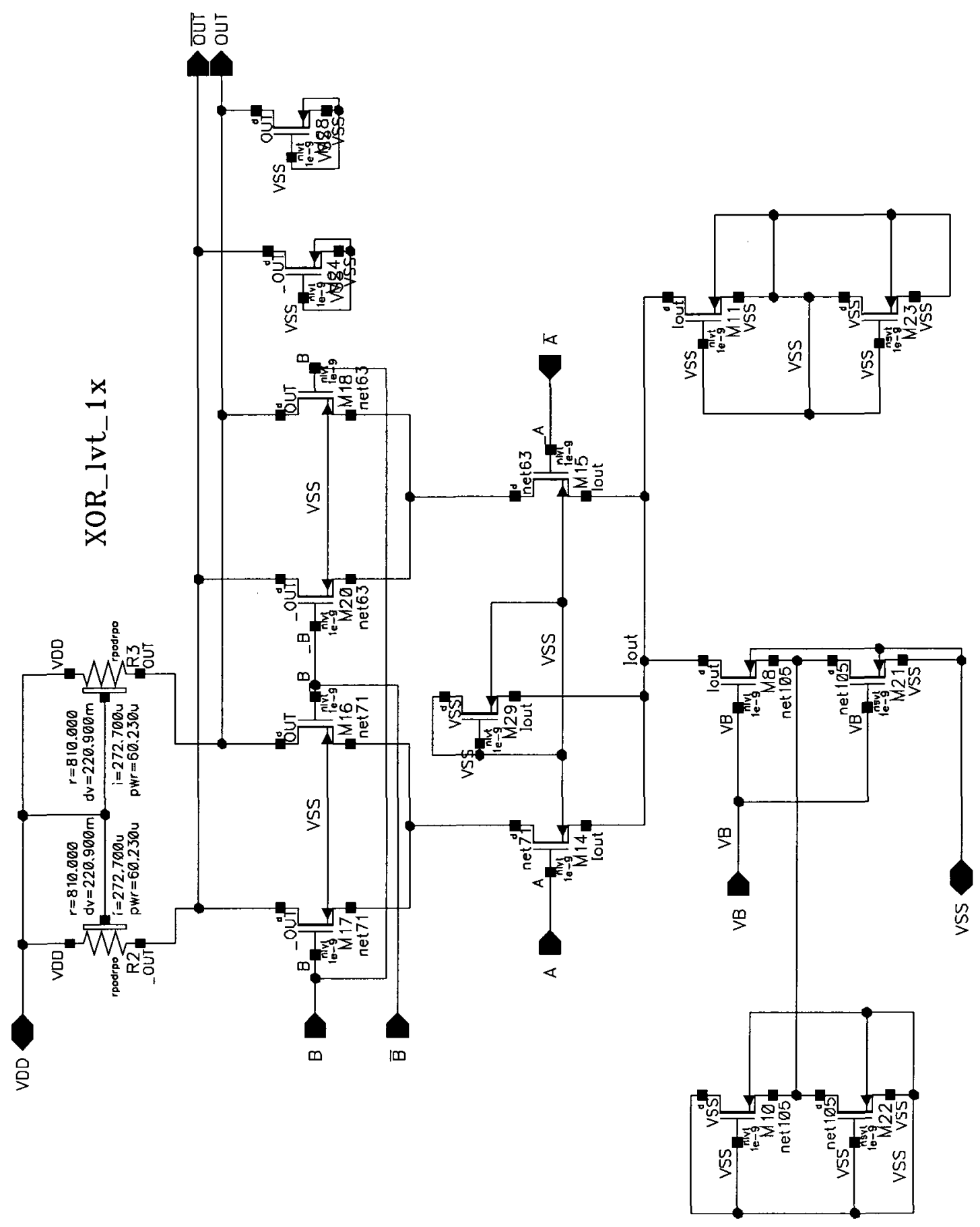

Figure A.2: Schematic of XOR 


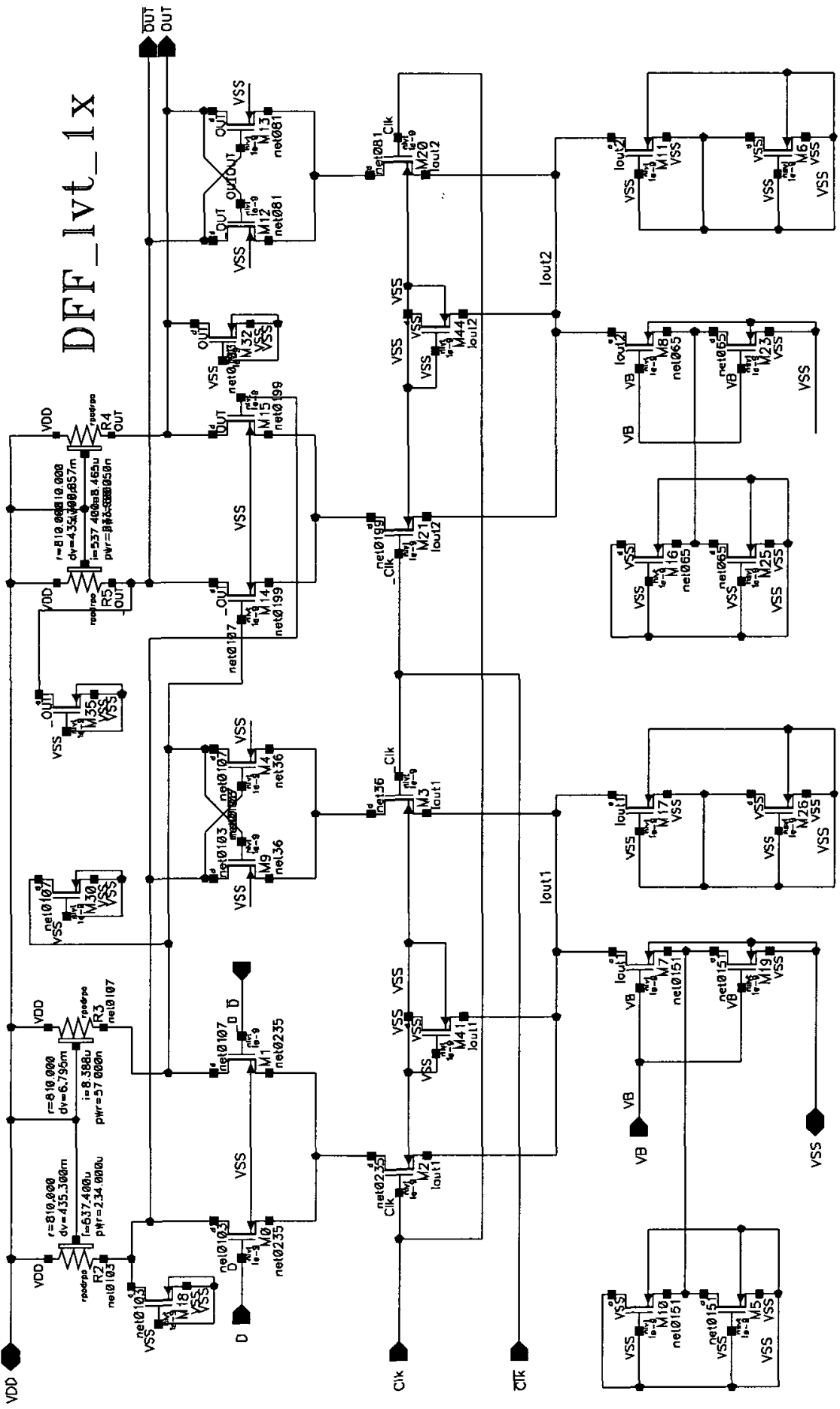

Figure A.3: Schematic of DFF 


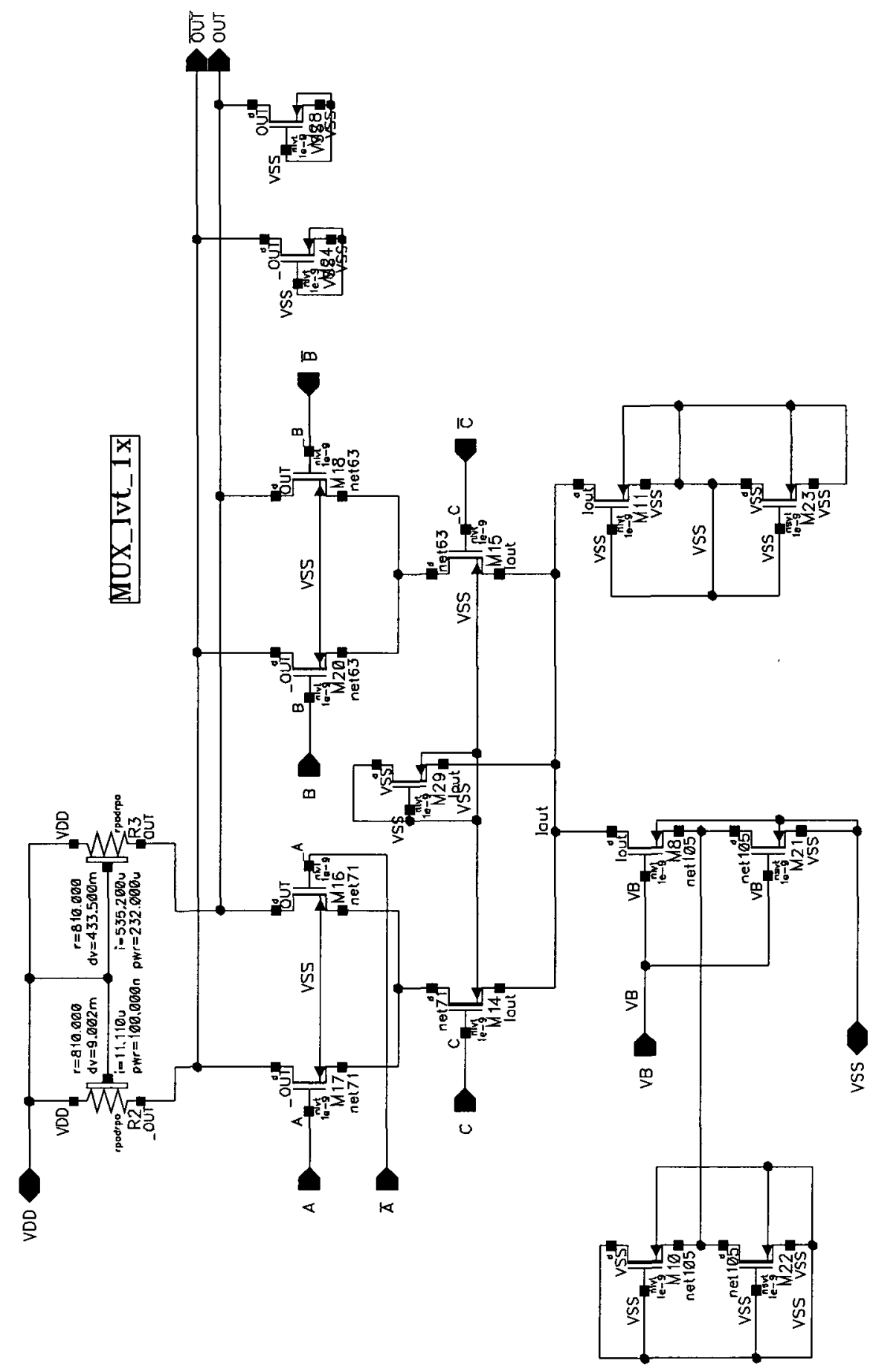

Figure A.4: Schematic of MUX 


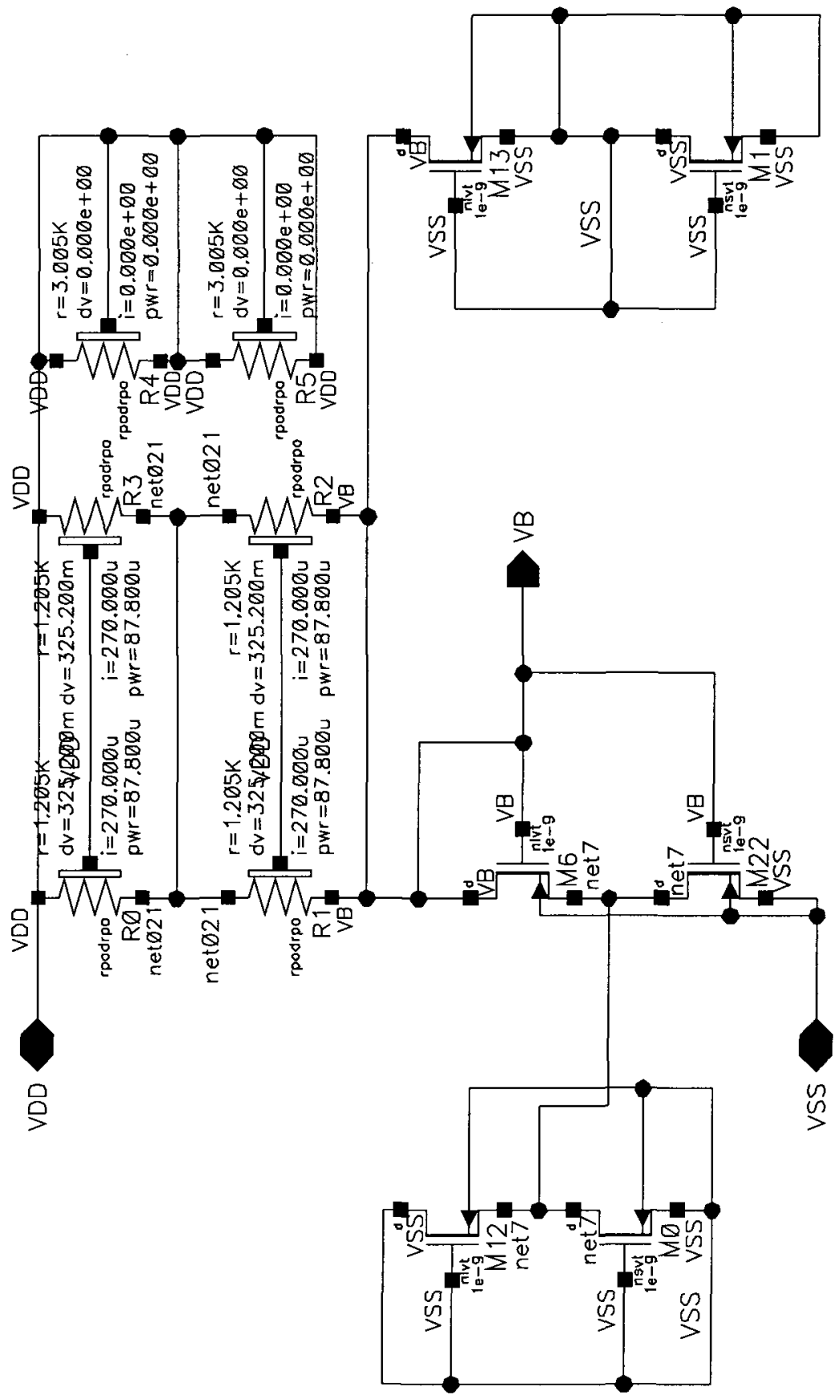

Figure A.5: Schematic of Current Mirror 


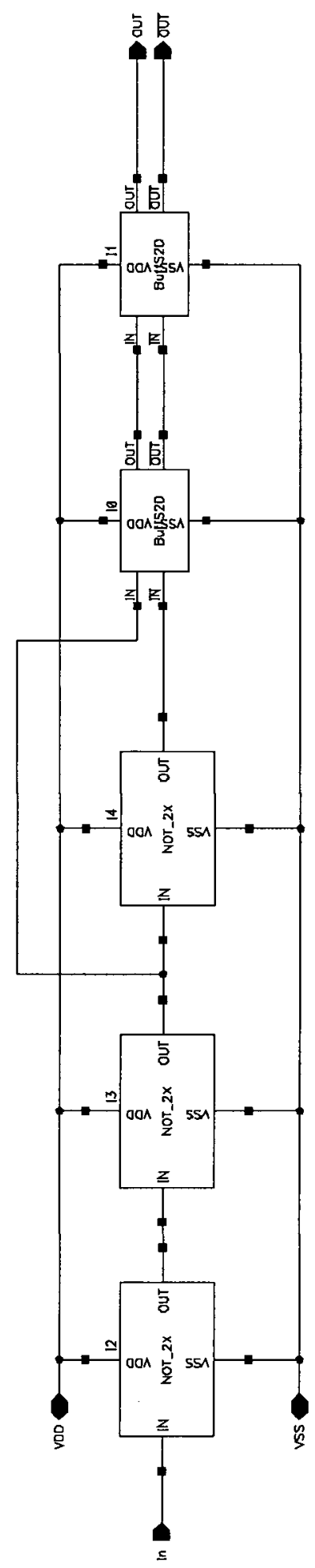

Figure A.6: Schematic of Single-to-Differential Converter 


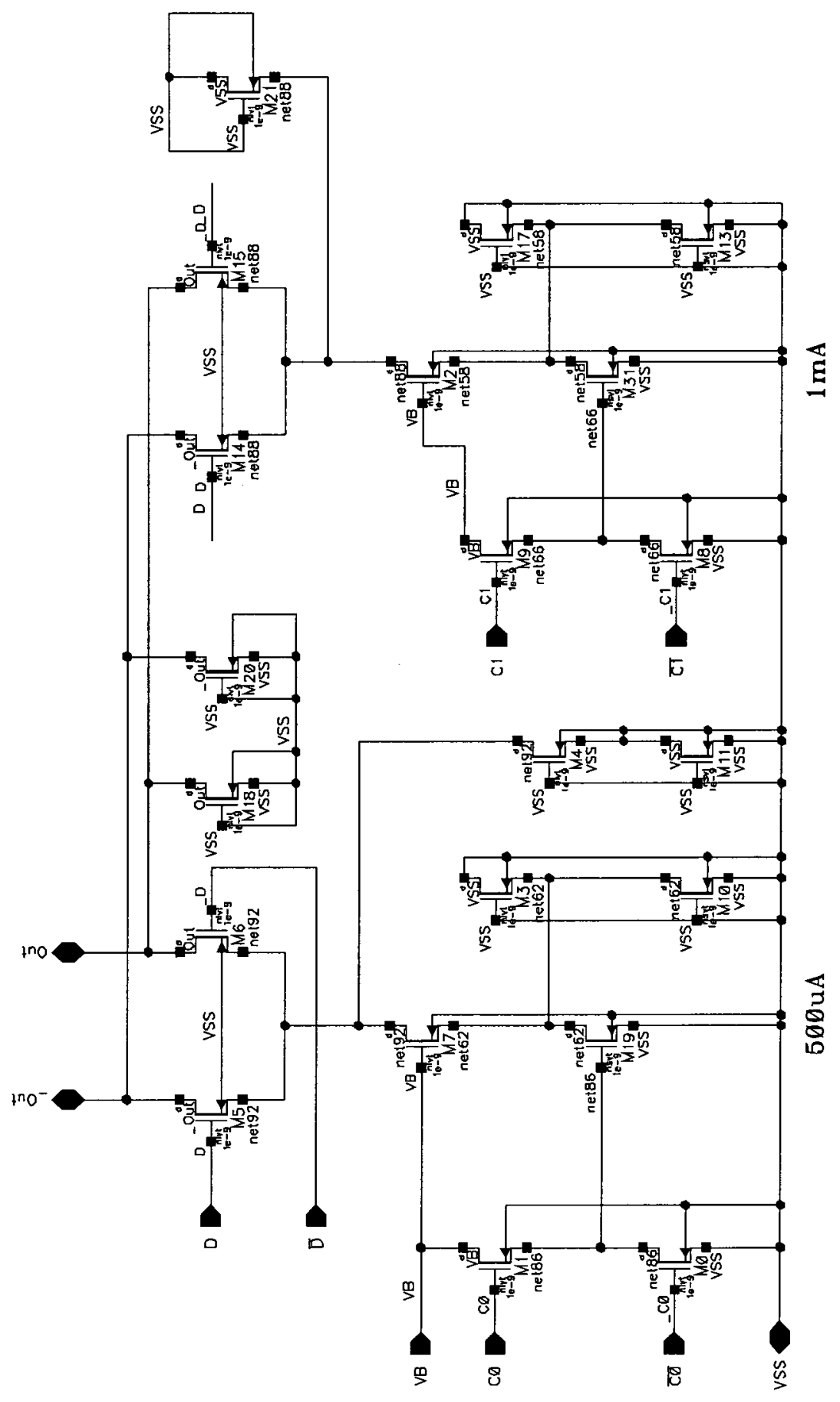

Figure A.7: Schematic of 2-Bit Driver 


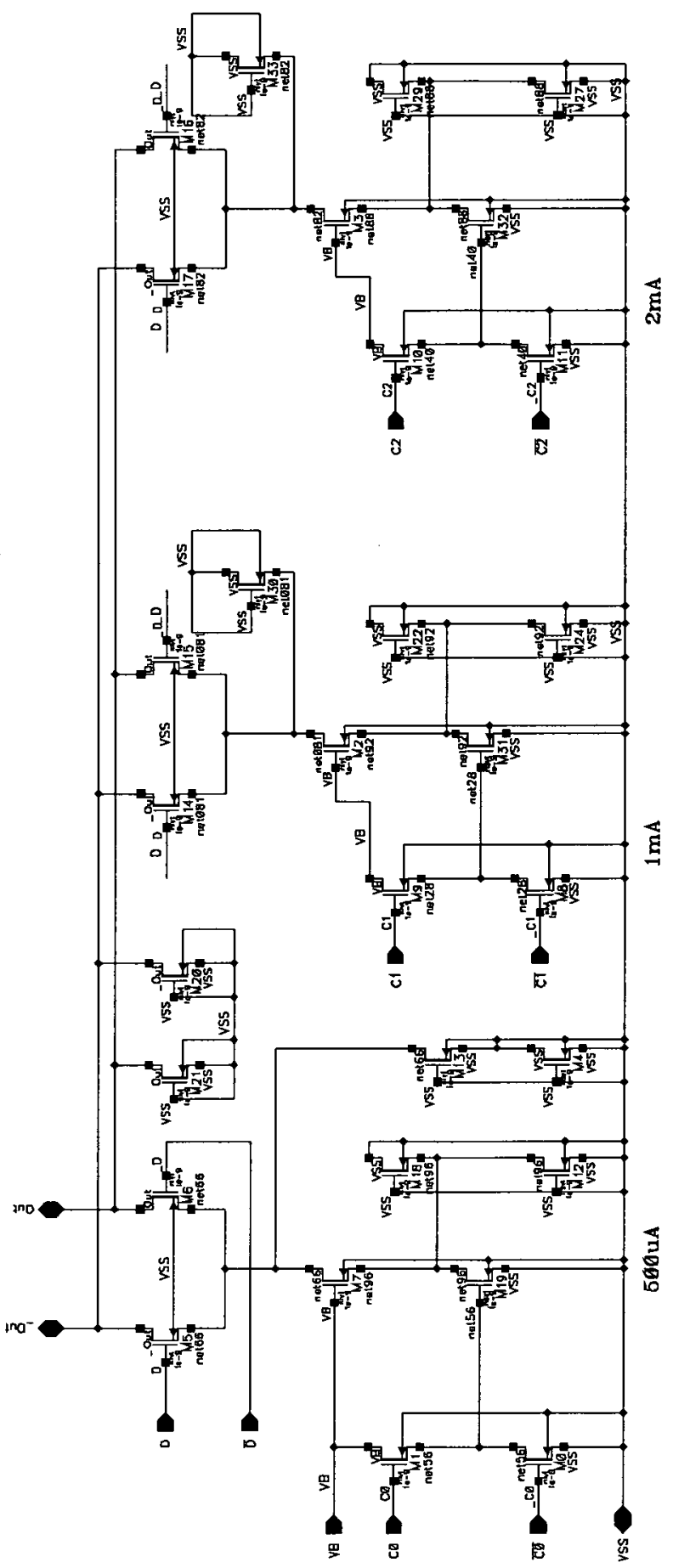

Figure A.8: Schematic of 3-Bit Driver 


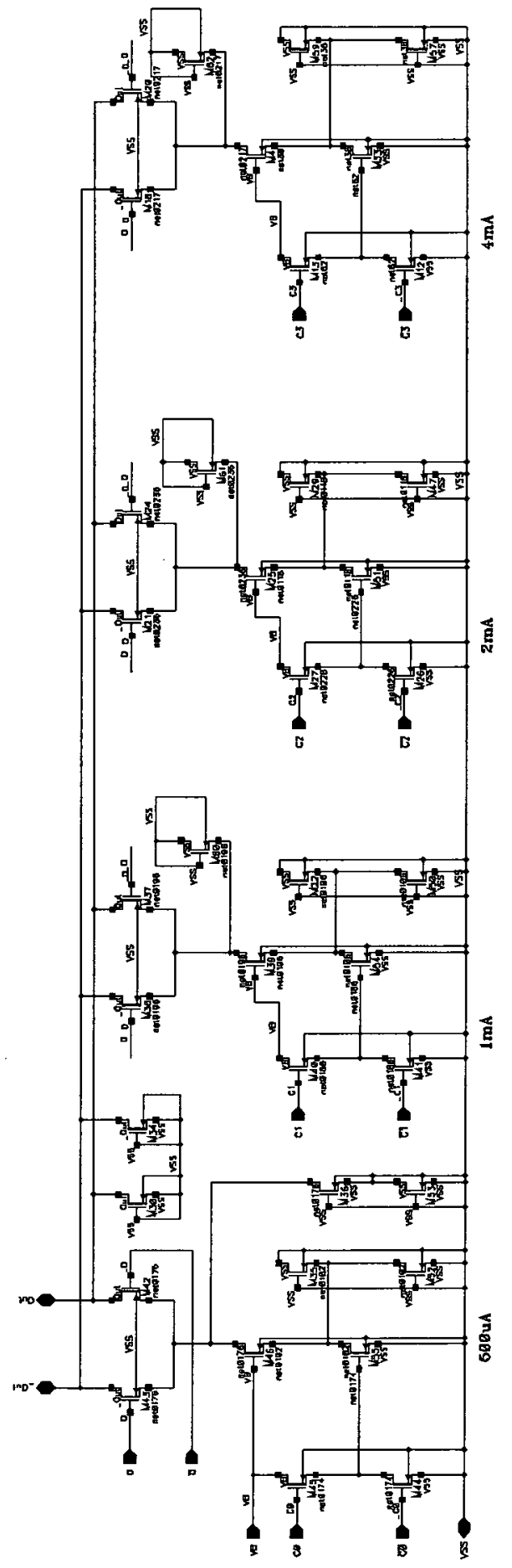

Figure A.9: Schematic of 4-Bit Driver 


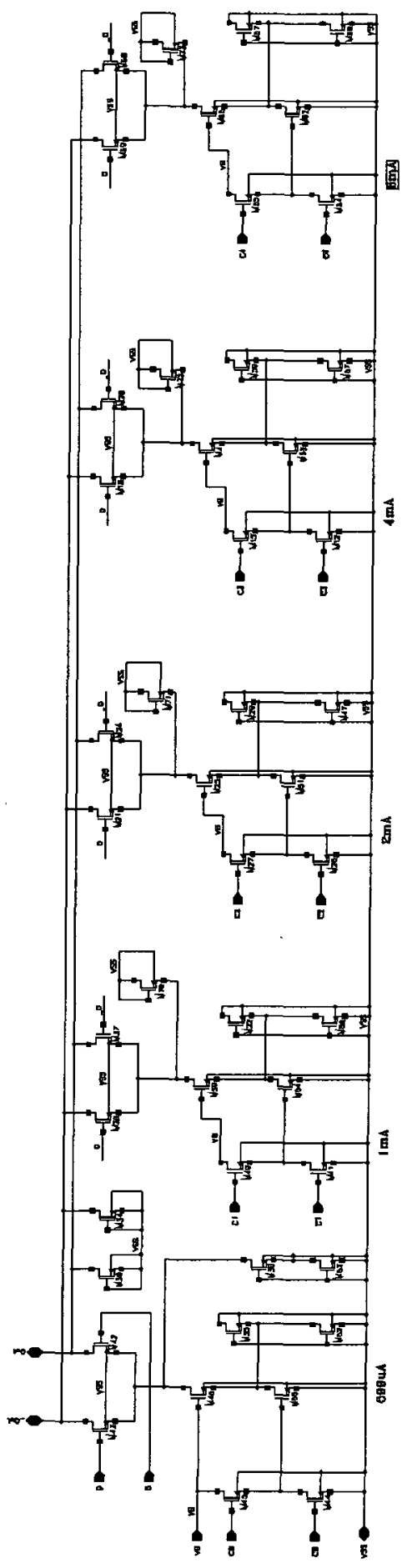

Figure A.10: Schematic of 5-Bit Driver 


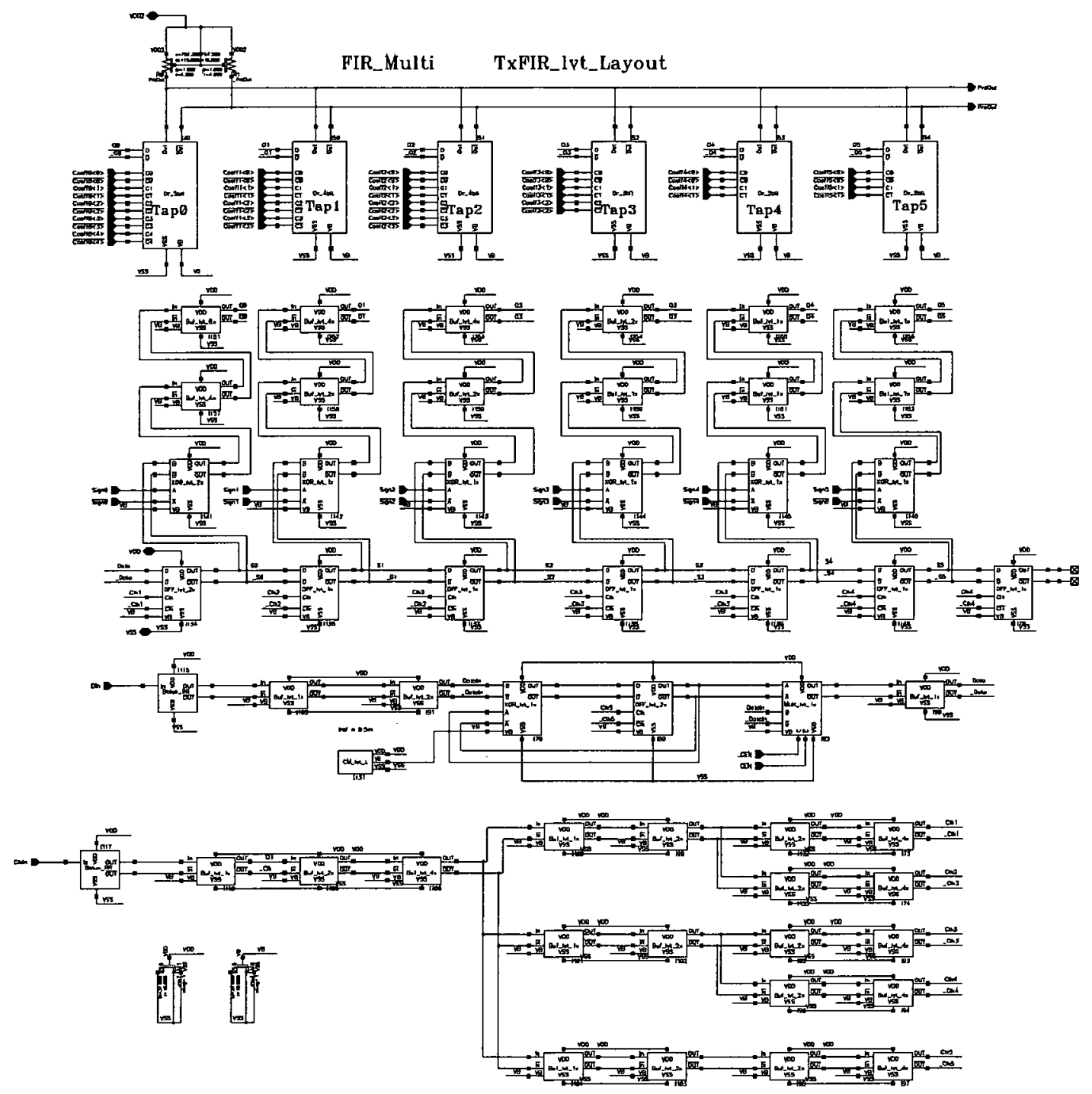

Figure A.11: Schematic of FIR 


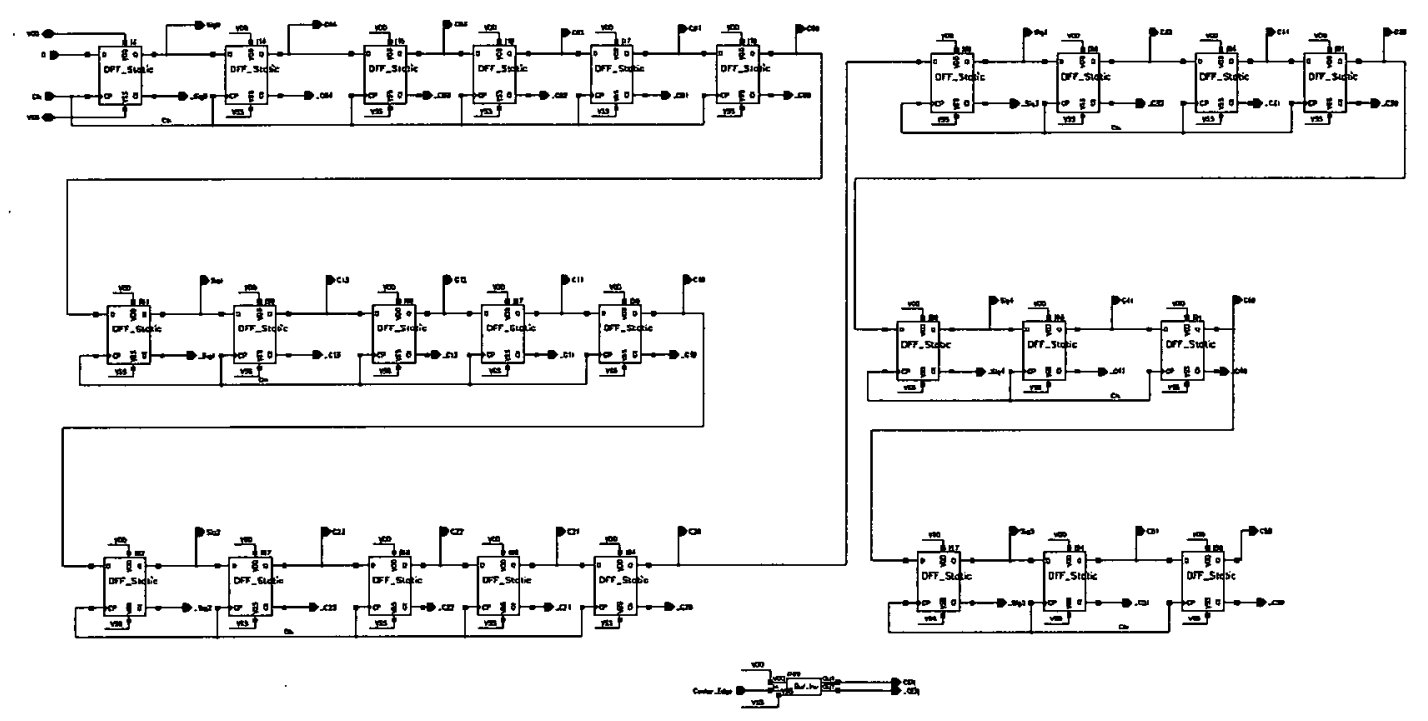

Figure A.12: Schematic of Coefficients Storage 


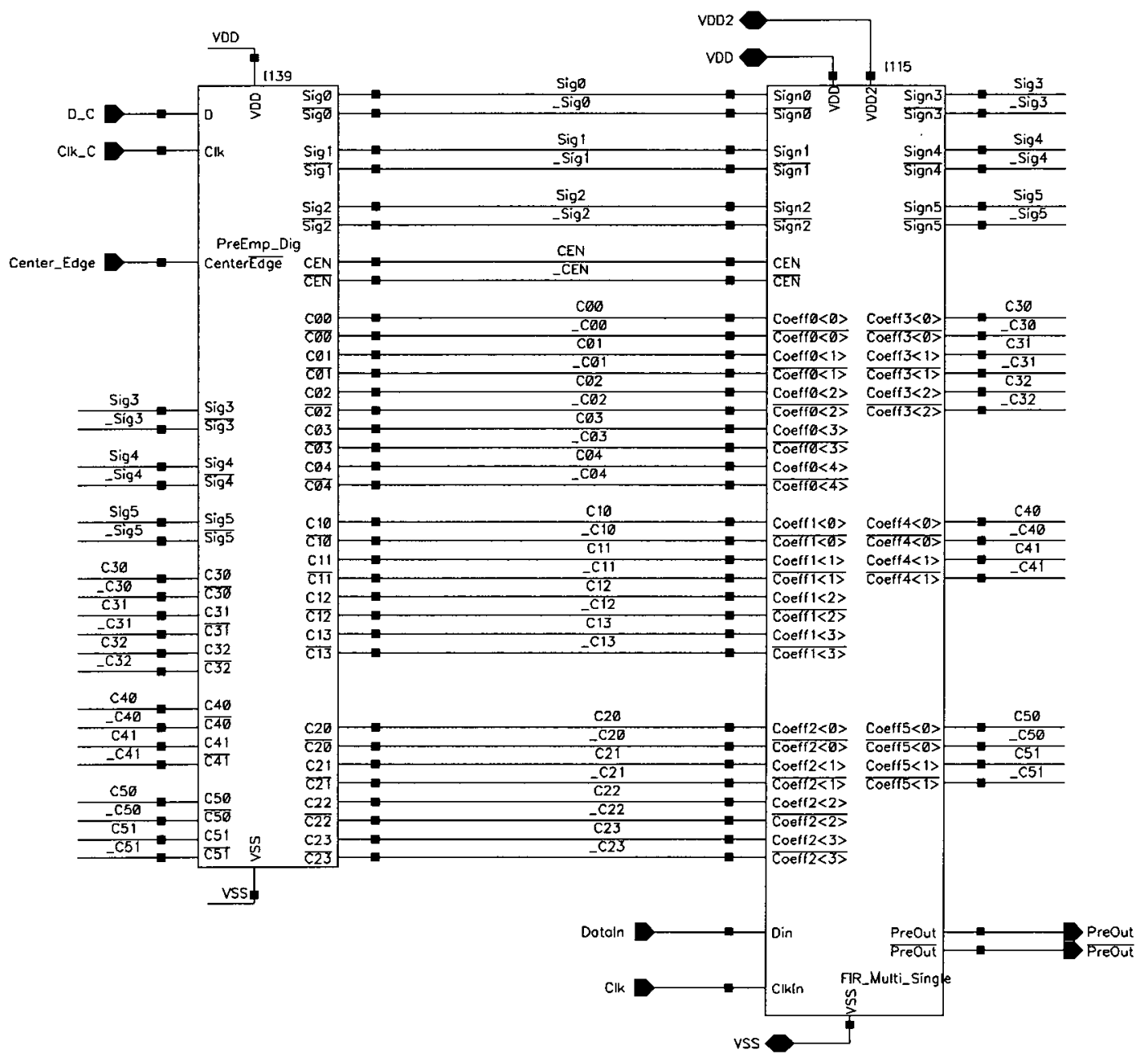

Figure A.13: Schematic of Pre-Emphasis Circuit 


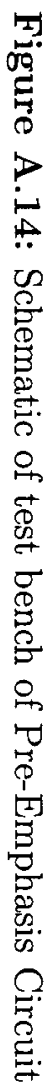

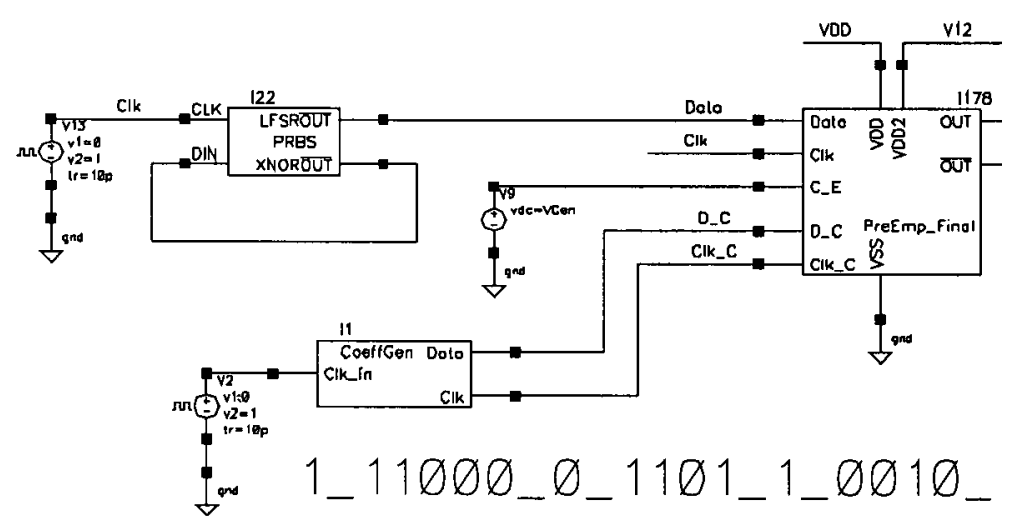




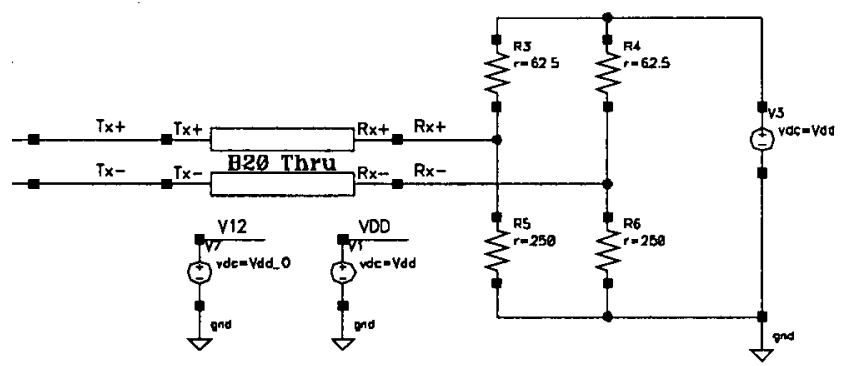

$1 \_\varnothing \varnothing \varnothing \_\varnothing \_\varnothing 1 \_1 \_\varnothing \emptyset$ 
Appendix B

\section{Layout of Pre-emphasis Circuits}

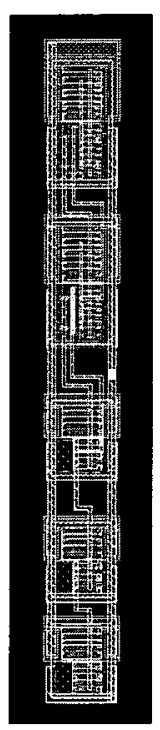

(a) Single-to-Differential Converter

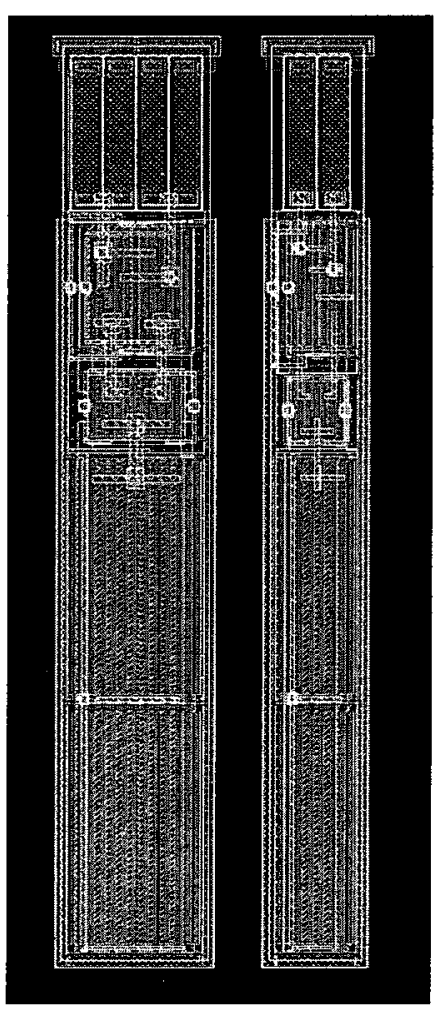

(b) XOR

Figure B.1: Layout of Single-to-Differential Converter and XOR 


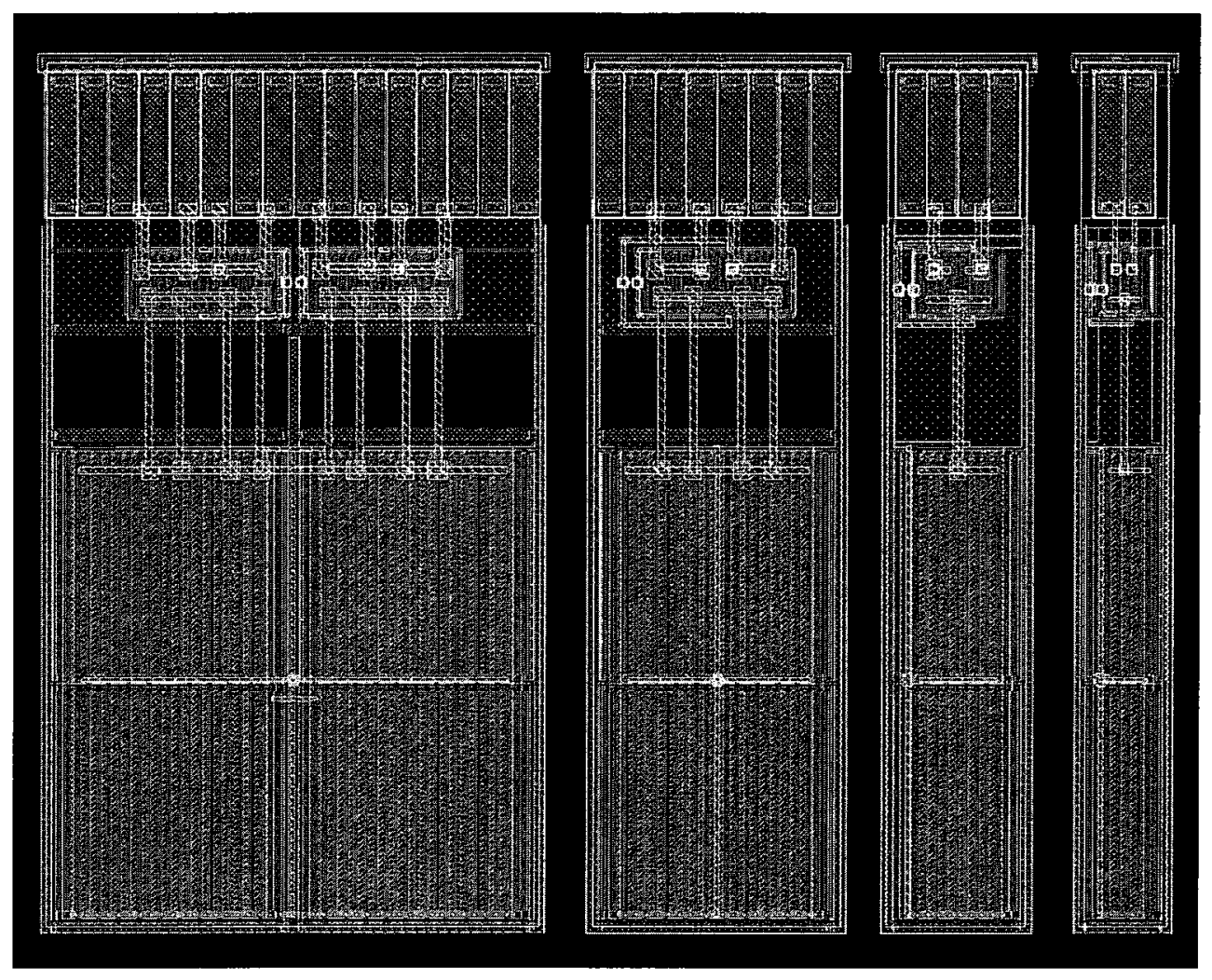

Figure B.2: Layout of Buffers 


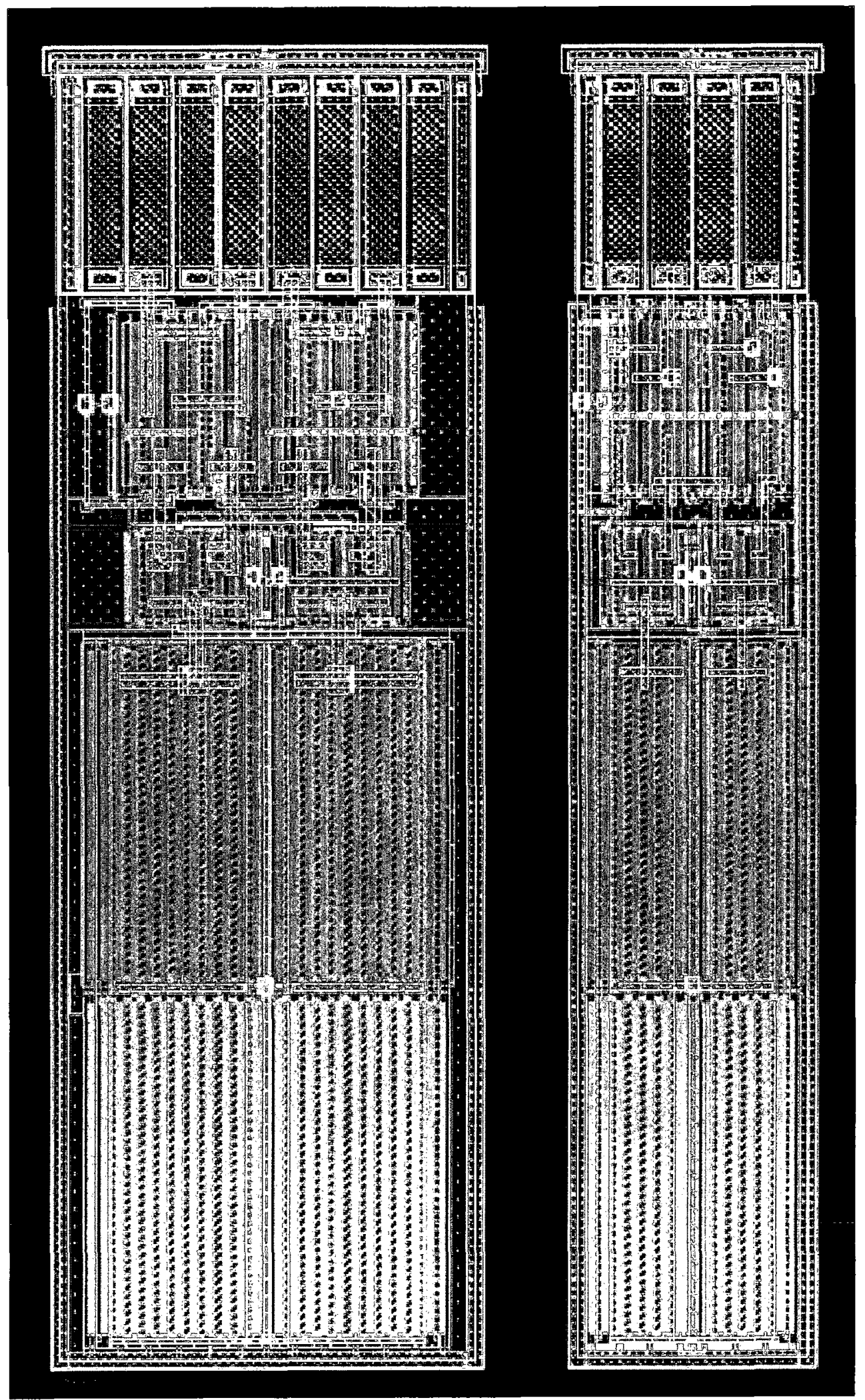

Figure B.3: Layout of DFFs 


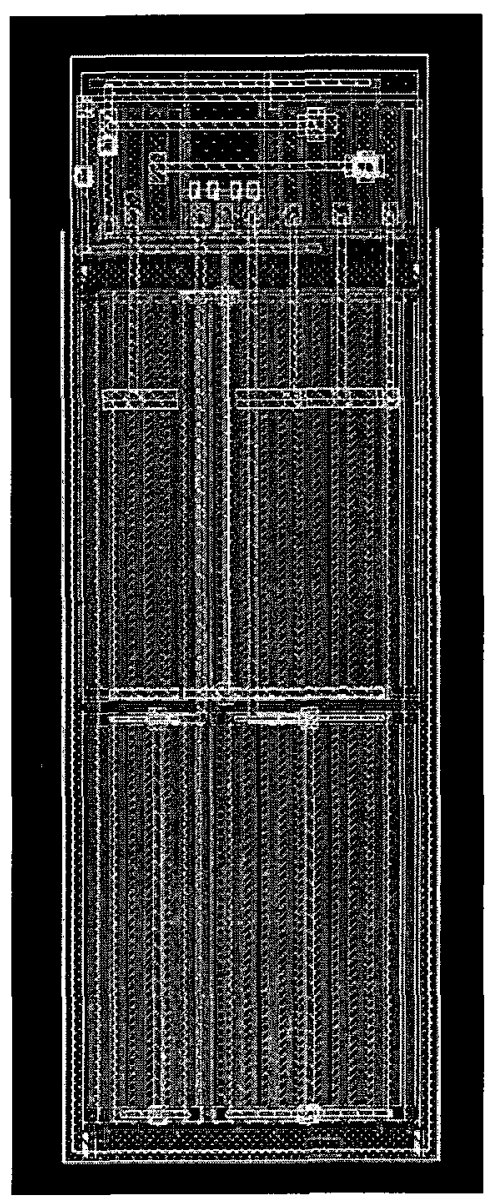

Figure B.4: Layout of 2-Bit Driver 


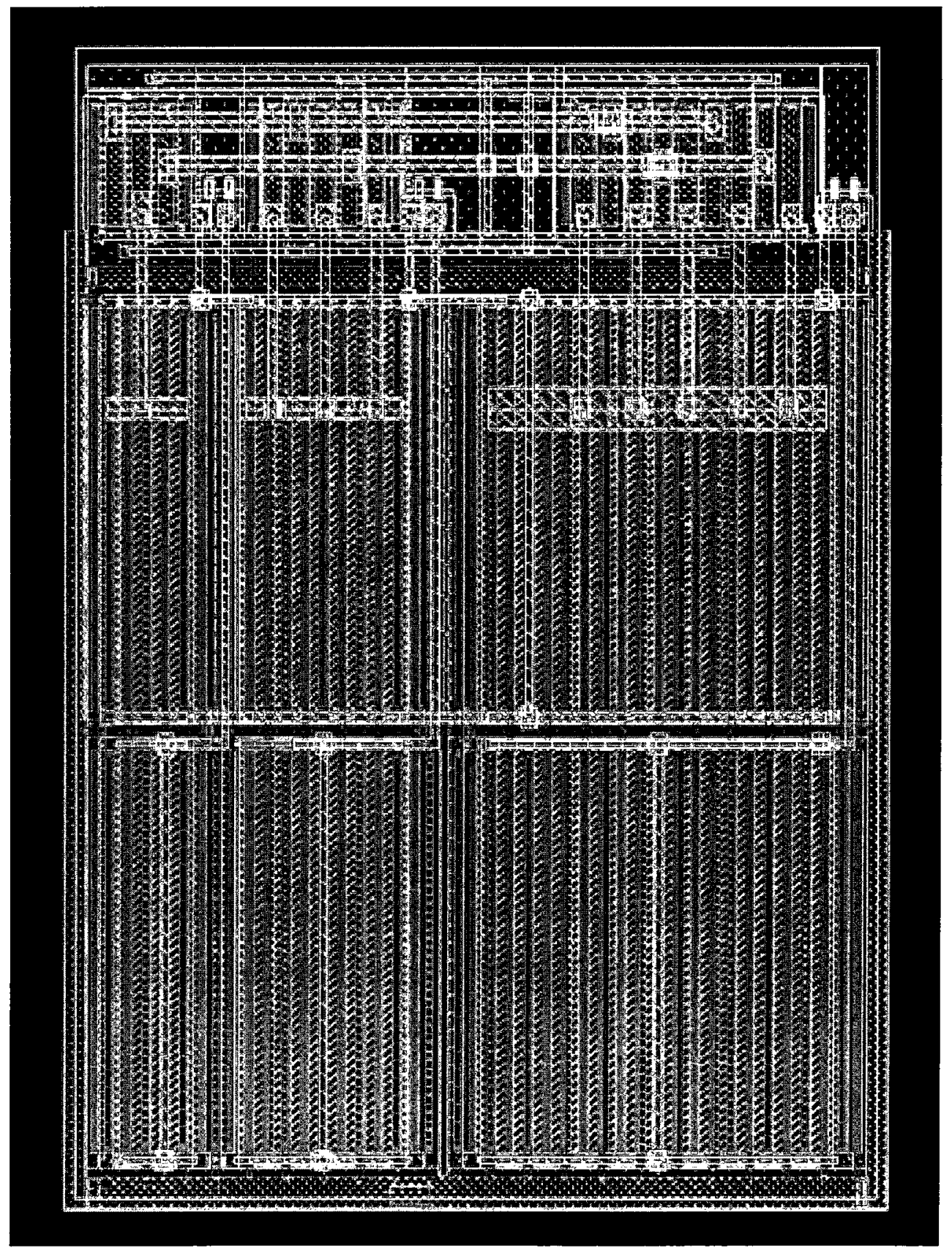

Figure B.5: Layout of 3-Bit Driver 


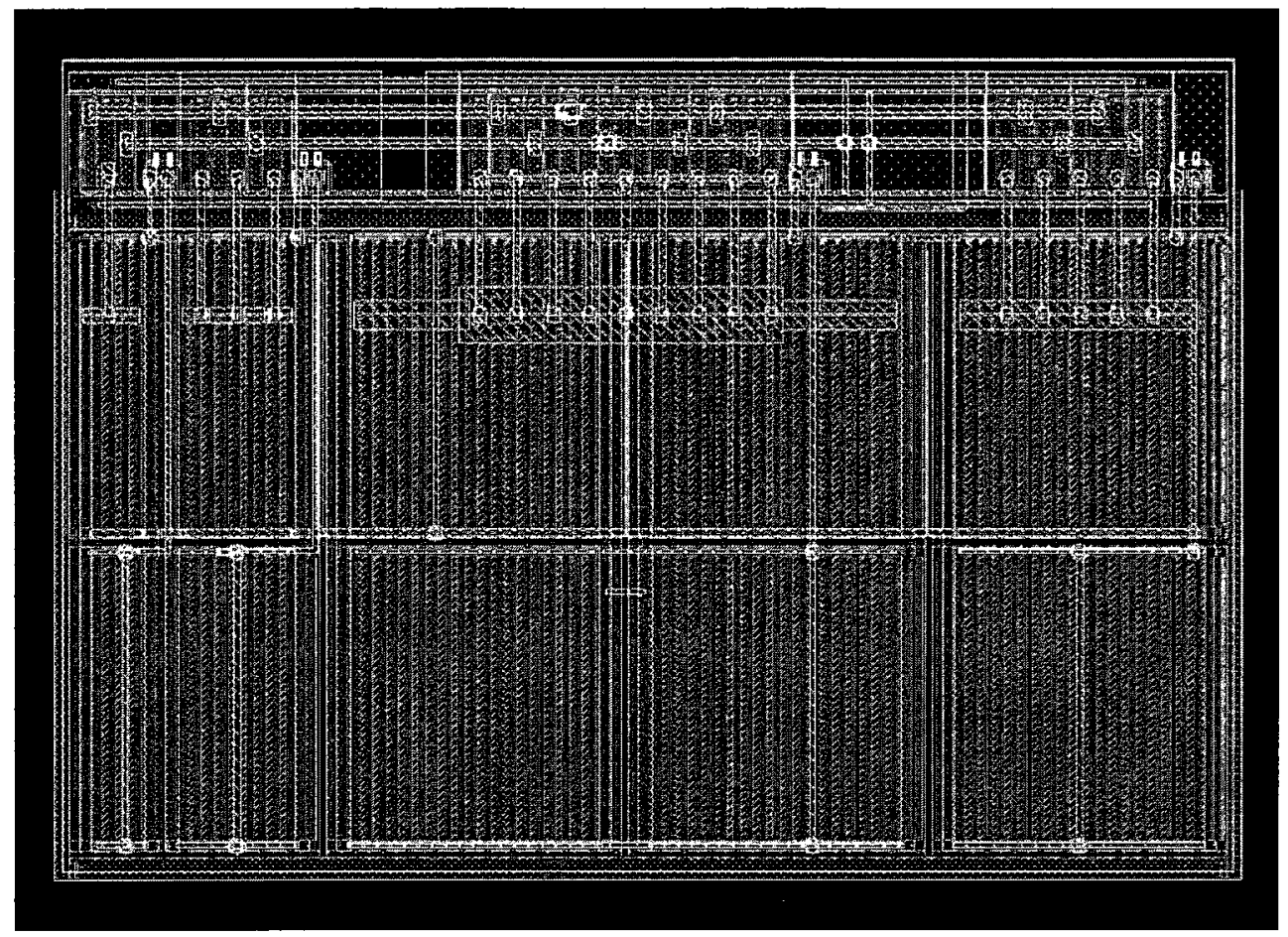

Figure B.6: Layout of 4-Bit Driver

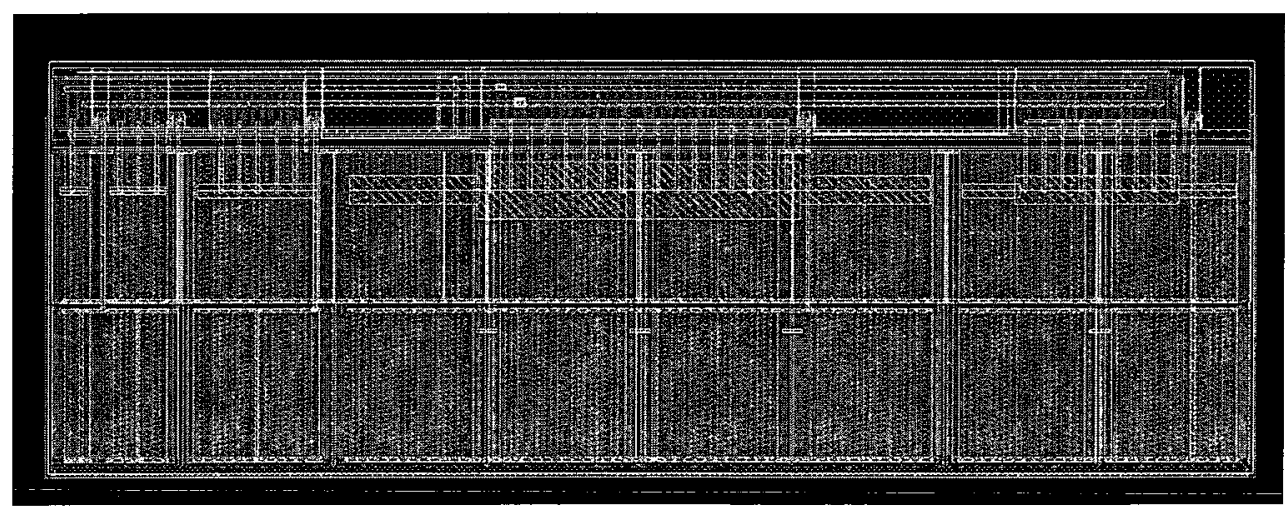

Figure B.7: Layout of 5-Bit Driver 


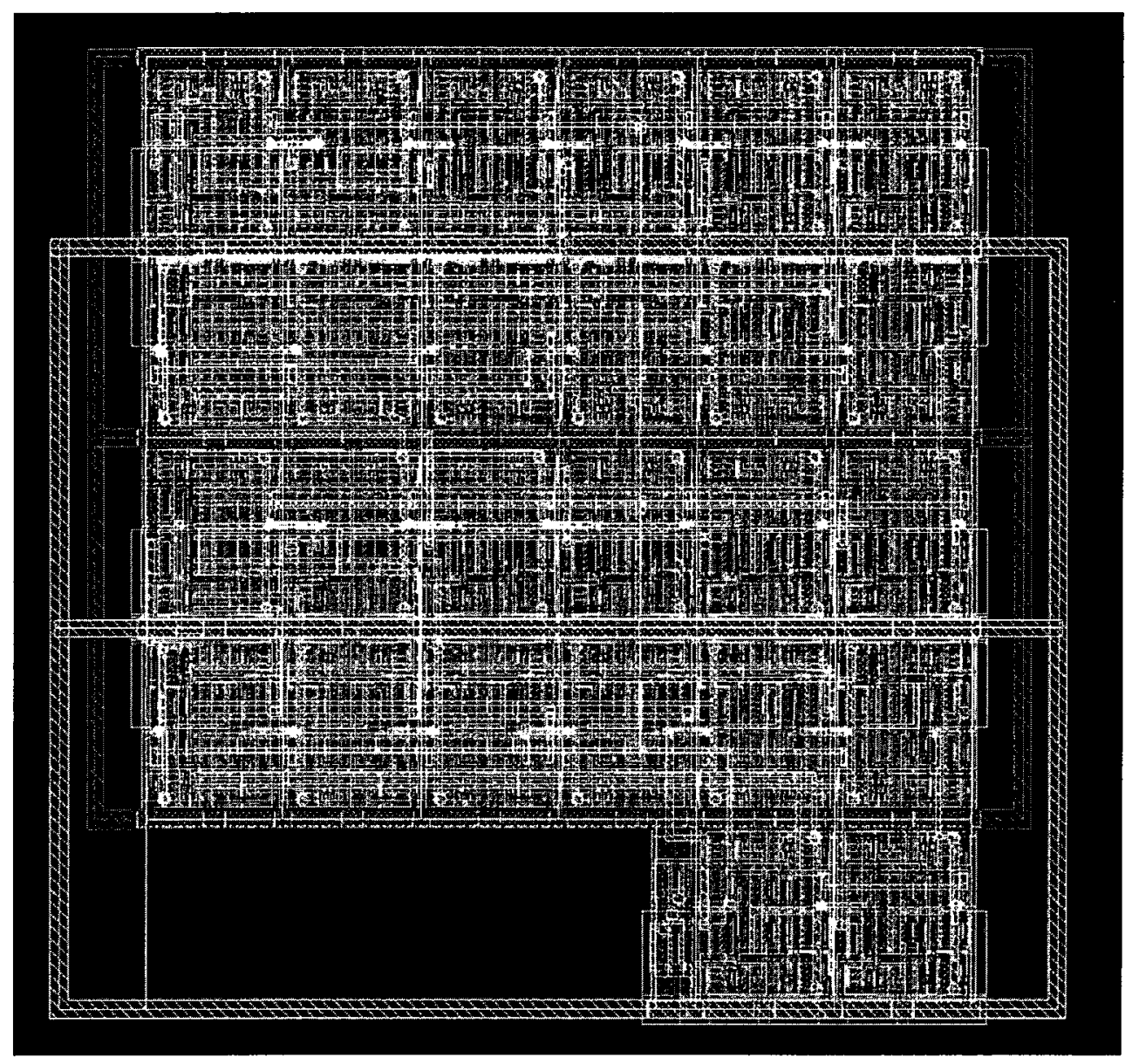

Figure B.8: Layout of Coefficients Storage 


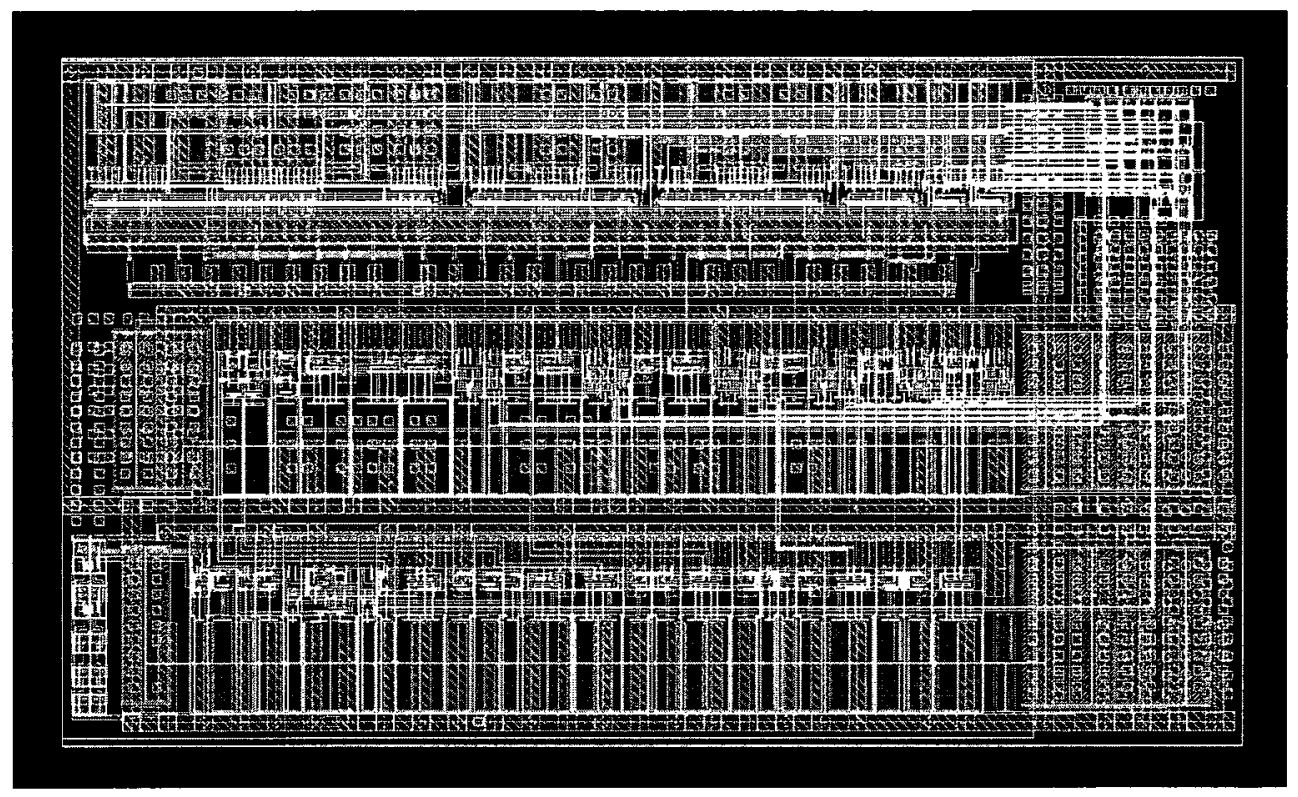

Figure B.9: Layout of Pre-Emphasis Circuit

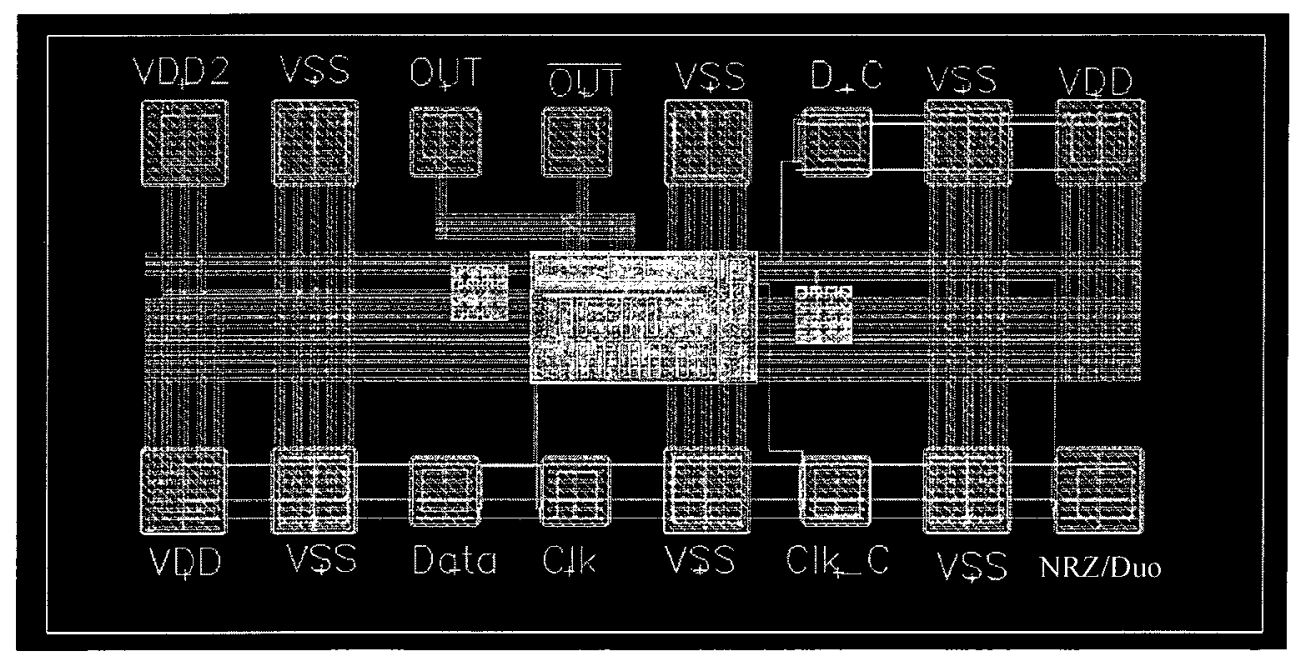

Figure B.10: Layout of Chip 